\title{
Rh-catalyzed Asymmetric Hydrogenation of Unsaturated Medium-Ring NH Lactams: Highly Enantioselective Synthesis of $\mathrm{N}$-Unprotected 2,3-dihydro-1,5-benzothiazepinones
}

Congcong Yin ${ }^{\dagger}, \S$, Tao Yang ${ }^{\dagger, \S}$, Yingmin Pan ${ }^{\dagger}$, Jialin Wen ${ }^{* \dagger, \star}$ and Xumu Zhang ${ }^{* \dagger}$

${ }^{\dagger}$ Shenzhen Grubbs Institute and Department of Chemistry, Southern University of Science and Technology, Shenzhen, Guangdong, 518055, China;

$¥$ Academy for Advanced Interdisciplinary Studies, Southern University of Science and Technology, Shenzhen, Guangdong, 518055, China.

[Supporting Information]

Contents

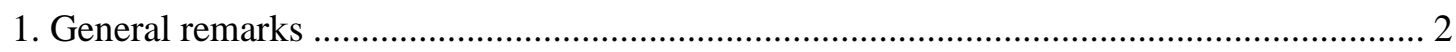

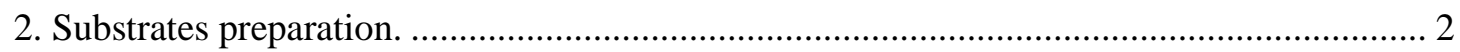

3. General procedure for asymmetric hydrogenation. ................................................... 10

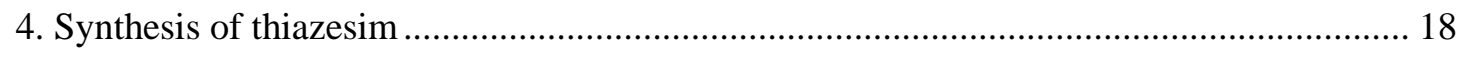

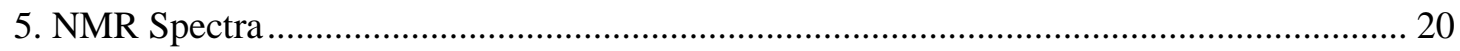

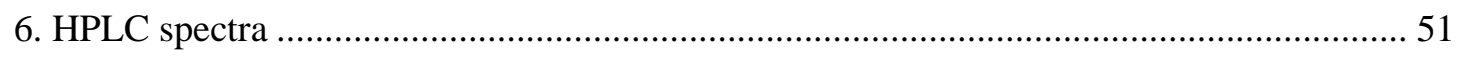

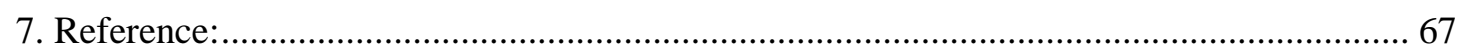




\section{General remarks}

Unless otherwise noted, all experiments were carried out under an atmosphere of argon in a glovebox or using standard Schlenk techniques. Solvents were dried with standard procedures and degassed with argon. Flash column chromatography was performed using Tsingdao silica gel (60, particle size 300-400 mesh). NMR spectra were recorded on a Bruker DPX 400 or a Bruker DPX 600 spectrometer. Chemical shifts $(\delta)$ are reported in ppm and respectively referenced to internal standard $\mathrm{Me}_{4} \mathrm{Si}$ and solvent signals $\left(\mathrm{Me}_{4} \mathrm{Si}, 0\right.$ ppm for ${ }^{1} \mathrm{H} \mathrm{NMR}$ in $\mathrm{CDCl}_{3}$; $77.0 \mathrm{ppm}$ in $\mathrm{CDCl}_{3}$ for $\left.{ }^{13} \mathrm{C} \mathrm{NMR}\right)$. Data are reported as: multiplicity ( $\mathrm{s}=$ singlet, $\mathrm{d}=$ doublet, $\mathrm{t}$ $=$ triplet, $\mathrm{q}=$ quartet, $\mathrm{m}=$ multiplet $)$, coupling constant in hertz $(\mathrm{Hz})$ and signal area integration in natural numbers. HPLC analysis was carried out with Agilent 1260 or 1290 Series instrument. Optical rotations were measured using a $1 \mathrm{~mL}$ cell with a $1 \mathrm{dm}$ path length on a Rudolph Autopol I polarimeter at $589 \mathrm{~nm}$. All new products were further characterized by HRMS. A positive ion mass spectrum of sample was acquired on a Thermo LTQ-FT mass spectrometer with an electrospray ionization source.

\section{Substrates preparation.}

\section{Procedure for preparation of aminothiophenol SM-1 ${ }^{1}$}

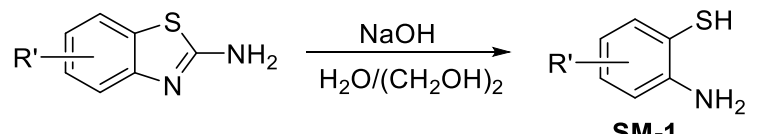

SM-1

A solution of 2-methylbenzothiazole derivative $(10 \mathrm{mmol})$ in a mixture of aqueous $50 \%$ $\mathrm{NaOH}$ and ethylene glycol $(\mathrm{v} / \mathrm{v}=1 / 1,20 \mathrm{~mL})$ was refluxed in an oil bath under argon atmosphere until the starting material disappeared. The mixture was then poured into ice-water, acidified to $\mathrm{pH}=3$ with $3 \mathrm{~N}$ aqueous $\mathrm{HCl}$, and extracted several times with $\mathrm{CH}_{2} \mathrm{Cl}_{2}$. The combined organic layers were washed with brine, dried over $\mathrm{Na}_{2} \mathrm{SO}_{4}$, and concentrated in vacuo. Purification by flash chromatography on silica using hexane/EtOAc (v/v $=2 / 1$ to $1 / 1)$ as an eluent gave the corresponding aminothiophenols SM-1.

Procedure for preparation of aryl alkynyl carboxylic acids SM-2 ${ }^{2}$<smiles>[R]c1ccc(I)cc1</smiles> 
The method for the synthesis of aryl alkyne carboxylic acids from aryl iodides was little modified from the method reported previously. A small round bottomed flask was charged with aryl iodide (5.0 mmol), DBU (1.83 g, 2.4 equiv., $12 \mathrm{mmol}), \mathrm{Pd}\left(\mathrm{PPh}_{3}\right)_{4}(144 \mathrm{mg}, 2.5 \mathrm{~mol} \%)$ and DMSO $6 \mathrm{~mL}$. The solution of propiolic acid (420 mg, 1.2 equiv., $6.0 \mathrm{mmol})$ in DMSO (6 mL) was poured to the flask. The mixture was stirred at ambient temperature for $12 \mathrm{~h}$. Upon completion of the reaction, the reaction mixture was diluted with ethyl acetate $(20 \mathrm{~mL})$, then filtered through a pad of Celite and concentrated, and poured into EtOAc $(50.0 \mathrm{~mL})$, and extracted with $\mathrm{NaHCO}_{3}$ (sat. aq). The aqueous layer was separated, acidified to $\mathrm{pH} 2.0$ by adding cold $\mathrm{HCl}(1 \mathrm{~N})$, and extracted with $\mathrm{CH}_{2} \mathrm{Cl}_{2}$. The combined organic layers were dried with $\mathrm{Na}_{2} \mathrm{SO}_{4}$, and the solvent was removed under reduced pressure. The resulting crude product was purified by flash chromatography (on silica, ethyl acetate: petroleum ether $=4: 1$ ) gave the corresponding aminothiophenols aryl alkynyl carboxylic acids SM-2.

\section{Preparation and characterization of substrates $1^{3}$}

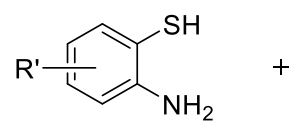

SM-1

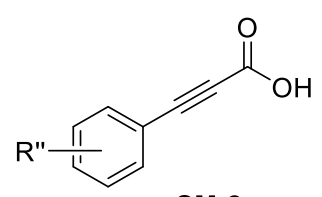

SM-2

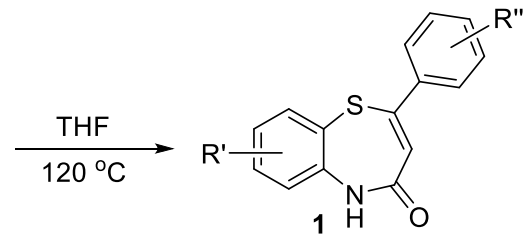

1

To a $50 \mathrm{~mL}$ Schlenk tube were added SM-1 $(10 \mathrm{mmol})$, SM-2 $(10 \mathrm{mmol})$ and THF $(5 \mathrm{~mL})$. The mixture was stirred at $120^{\circ} \mathrm{C}$ in an oil bath under argon for $12 \mathrm{~h}$. After the reaction mixture was cooled down to room temperature, $\mathrm{Et}_{2} \mathrm{O}(30 \mathrm{~mL})$ was added. The yellow precipitate was collected by filtration to give the product 1 . All the $N$-unprotected substrates were prepared by this method.
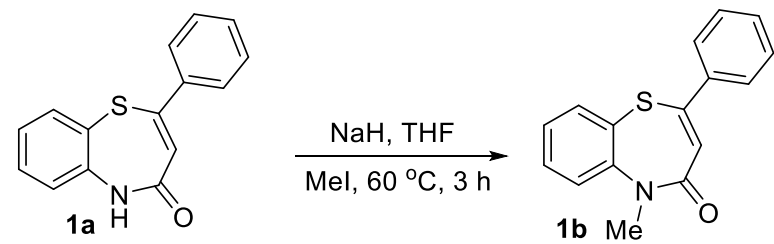

In a $50 \mathrm{~mL}$ Schlenk tube equipped with $1 \mathbf{a}(253 \mathrm{mg}, 1.0 \mathrm{mmol})$ and THF $(5 \mathrm{~mL})$ was added $\mathrm{NaH}(60 \%)(48 \mathrm{mg}, 1.2 \mathrm{mmol})$ at $0{ }^{\circ} \mathrm{C}$. After $0.5 \mathrm{~h}$, the mixture became a clear solution, and MeI (213 mg, $1.5 \mathrm{mmol}$ ) was added. After stirring at $60{ }^{\circ} \mathrm{C}$ (in an oil bath) for $3 \mathrm{~h}$ (monitored by TLC), the reaction was quenched by addition of saturated aqueous $\mathrm{NH}_{4} \mathrm{Cl}$ solution. The reaction mixture was extracted with $\mathrm{CH}_{2} \mathrm{Cl}_{2}(3 \times 30 \mathrm{~mL})$, the combined organic layers were 
dried over $\mathrm{Na}_{2} \mathrm{SO}_{4}$, evaporated in vacuo, and further purified by flash chromatography on silica (petroleum ether / ethyl acetate $=5 / 1$ ) to give pure compound $\mathbf{1 b}$ (yellow solid, $227 \mathrm{mg}, 85 \%$ yield).

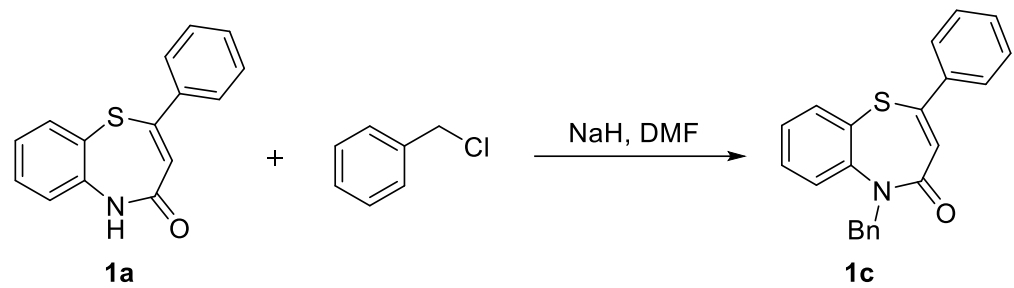

$\mathrm{NaH}(60 \%)(30 \mathrm{mg}, 0.75 \mathrm{mmol})$ was added to a mixture of 2-phenylbenzo[b][1,4]thiazepin4(5H)-one 1a (126 mg, $0.5 \mathrm{mmol})$ in DMF $(2 \mathrm{~mL})$ at $0{ }^{\circ} \mathrm{C}$. After stirring at room temperature for $0.5 \mathrm{~h}$, the mixture became a clear solution, and (chloromethyl)benzene (128 $\mathrm{mg}, 0.75 \mathrm{mmol})$ was added. After strirring at $25^{\circ} \mathrm{C}$ for $10 \mathrm{~h}$ (monitored by TLC), the reaction was quenched by the addition of sat. aqueous $\mathrm{NH}_{4} \mathrm{Cl}$ solution. The reaction mixture was extracted with $\mathrm{CH}_{2} \mathrm{Cl}_{2}$ $(3 \times 50 \mathrm{~mL})$. The combined organic layers were dried over $\mathrm{Na}_{2} \mathrm{SO}_{4}$, evaporated in vacuo, and further purified by flash chromatography on silica (petroleum ether $/$ ethyl acetate $=2 / 1$ ) to give pure compound 1c (yellow solid, $158 \mathrm{mg}, 92 \%$ yield).

The characterization data of compounds $\mathbf{1 a}$ and $\mathbf{1 b}$ are in accordance with the reported data in the literature. ${ }^{3}$ The characterization data of compounds $\mathbf{1 0}$ are in accordance with the reported data in the literature. ${ }^{4}$

\section{2-Phenylbenzo $[b][1,4]$ thiazepin-4(5H)-one (1a)}<smiles>O=C1C=C(c2ccccc2)Sc2ccccc2N1</smiles>

Yellow solid, $1.14 \mathrm{~g}, 45 \%$ yield, ${ }^{1} \mathrm{H}$ NMR (400 MHz, $\left.\mathrm{CDCl}_{3}\right) \delta 9.29(\mathrm{~s}, 1 \mathrm{H})$, $7.80-7.78(\mathrm{~m}, 2 \mathrm{H}), 7.58(\mathrm{dd}, J=7.8,1.5 \mathrm{~Hz}, 1 \mathrm{H}), 7.42-7.39(\mathrm{~m}, 3 \mathrm{H}), 7.37(\mathrm{dd}, J=7.6,1.5$ $\mathrm{Hz}, 1 \mathrm{H}), 7.22(\mathrm{dd}, J=8.0,1.4 \mathrm{~Hz}, 1 \mathrm{H}), 7.18(\mathrm{td}, J=7.5,1.4 \mathrm{~Hz}, 1 \mathrm{H}), 6.51(\mathrm{~d}, J=1.7 \mathrm{~Hz}, 1 \mathrm{H})$. ${ }^{13} \mathrm{C}$ NMR $\left(100 \mathrm{MHz}, \mathrm{CDCl}_{3}\right) \delta 169.6,152.4,139.9,138.2,132.8,130.3,130.2,128.6,128.4$, $128.0,125.8,123.9,122.7 \mathrm{ppm}$. 


\section{5-Methyl-2-phenylbenzo $[b][1,4]$ thiazepin-4(5H)-one (1b)}

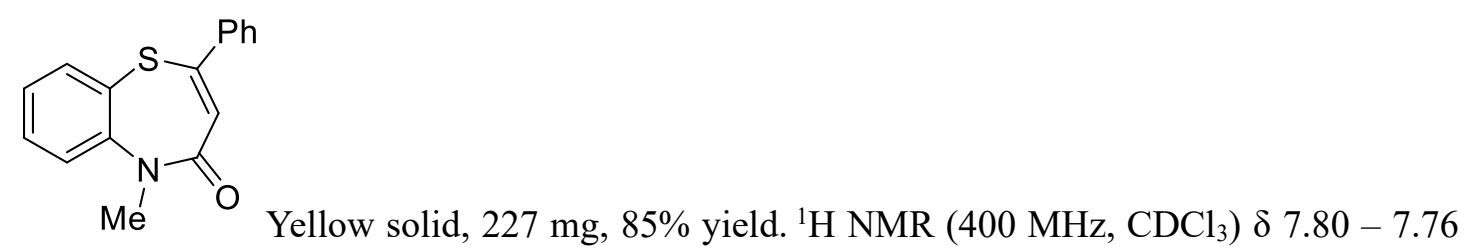

(m, 2H), $7.64(\mathrm{dd}, J=7.8,1.5 \mathrm{~Hz}, 1 \mathrm{H}), 7.44-7.37(\mathrm{~m}, 4 \mathrm{H}), 7.32(\mathrm{dd}, J=8.1,1.4 \mathrm{~Hz}, 1 \mathrm{H})$, $7.19(\mathrm{td}, J=7.5,1.4 \mathrm{~Hz}, 1 \mathrm{H}), 6.46(\mathrm{~s}, 1 \mathrm{H}), 3.55(\mathrm{~s}, 3 \mathrm{H}) .{ }^{13} \mathrm{C} \mathrm{NMR}\left(100 \mathrm{MHz}, \mathrm{CDCl}_{3}\right) \delta 168.0$, $151.7,145.1,137.9,133.9,133.1,130.1,129.9,128.6,127.9,125.9,124.2,124.1,37.8$ ppm.

\section{5-Benzyl-2-phenylbenzo $[b][1,4]$ thiazepin-4(5H)-one (1c)}<smiles>O=C1C=C(c2ccccc2)Sc2ccccc2N1Cc1ccccc1</smiles>

Yellow solid, $158 \mathrm{mg}, 92 \%$ yield. ${ }^{1} \mathrm{H}$ NMR $\left(400 \mathrm{MHz}, \mathrm{CDCl}_{3}\right) \delta 7.80-7.76$ (m, 2H), $7.61(\mathrm{dd}, J=7.7,1.0 \mathrm{~Hz}, 1 \mathrm{H}), 7.40-7.35(\mathrm{~m}, 5 \mathrm{H}), 7.33-7.28(\mathrm{~m}, 5 \mathrm{H}), 7.23-7.19$ (m, 1H), $7.14-7.10(\mathrm{~m}, 1 \mathrm{H}), 6.51(\mathrm{~s}, 1 \mathrm{H}), 5.63(\mathrm{~d}, J=15.4 \mathrm{~Hz}, 1 \mathrm{H}), 4.97(\mathrm{~d}, J=15.4 \mathrm{~Hz}$, 1H). ${ }^{13} \mathrm{C}$ NMR (100 MHz, $\left.\mathrm{CDCl}_{3}\right) \delta 168.0,151.7,143.6,137.8,136.8,135.5,133.3,130.1$, $129.7,128.6,128.5,127.8,127.7,127.2,126.0,125.1,123.9,53.1 \mathrm{ppm}$. HRMS (ESI) Calculated for $\mathrm{C}_{22} \mathrm{H}_{17} \mathrm{NNaOS}[\mathrm{M}+\mathrm{Na}]^{+}$366.0923; found 366.0917.

7-Fluoro-2-phenylbenzo[b][1,4]thiazepin-4(5H)-one (1d)<smiles>O=C1C=C(c2ccccc2)Sc2ccc(F)cc2N1</smiles>

Yellow solid, $1.06 \mathrm{~g}, 39 \%$ yield, ${ }^{1} \mathrm{H}$ NMR (400 MHz, DMSO) $\delta 10.55$ (s, 1H), $7.81-7.79(\mathrm{~m}, 2 \mathrm{H}), 7.60(\mathrm{dd}, J=8.3,2.6 \mathrm{~Hz}, 1 \mathrm{H}), 7.52-7.39(\mathrm{~m}, 3 \mathrm{H}), 7.38-7.22(\mathrm{~m}$, 2H), $6.56(\mathrm{~s}, 1 \mathrm{H}) .{ }^{13} \mathrm{C}$ NMR (150 MHz, DMSO) $\delta 167.5,159.0(\mathrm{~d}, J=245.0 \mathrm{~Hz}), 148.2,137.8$, $137.4(\mathrm{~d}, J=1.7 \mathrm{~Hz}), 130.7,130.0(\mathrm{~d}, J=8.7 \mathrm{~Hz}), 129.3,127.9,126.0,124.7(\mathrm{~d}, J=8.5 \mathrm{~Hz})$, $119.6(\mathrm{~d}, J=23.5 \mathrm{~Hz}), 117.6(\mathrm{~d}, J=22.6 \mathrm{~Hz})$. HRMS (ESI) Calculated for $\mathrm{C}_{15} \mathrm{H}_{10} \mathrm{FNNaOS}$ $[\mathrm{M}+\mathrm{Na}]^{+}$294.0359; found 294.0364. 
<smiles>O=C1C=C(c2ccccc2)Sc2ccc(Cl)cc2N1</smiles>

Yellow solid, $1.01 \mathrm{~g}, 35 \%$ yield, ${ }^{1} \mathrm{H}$ NMR (400 MHz, DMSO) $\delta 10.62$ (s, 1H), $7.82-7.77(\mathrm{~m}, 2 \mathrm{H}), 7.68(\mathrm{~d}, J=8.2 \mathrm{~Hz}, 1 \mathrm{H}), 7.48-7.46(\mathrm{~m}, 3 \mathrm{H}), 7.29-7.25(\mathrm{~m}, 2 \mathrm{H})$, $6.58(\mathrm{~s}, 1 \mathrm{H}) \cdot{ }^{13} \mathrm{C}$ NMR (100 MHz, DMSO) $\delta 167.5,148.5,142.2,137.6,134.8,134.6,130.8$, 129.3, 127.9, 126.8, 125.9, 125.3, 122.7. HRMS (ESI) Calculated for $\mathrm{C}_{15} \mathrm{H}_{10} \mathrm{ClNNaOS}$ $[\mathrm{M}+\mathrm{Na}]^{+}$310.0064; found 310.0068.

\section{7-Methyl-2-phenylbenzo[b][1,4] thiazepin-4(5H)-one (1f)}<smiles>Cc1ccc2c(c1)NC(=O)C=C(c1ccccc1)S2</smiles>

Yellow solid, $1.12 \mathrm{~g}, 42 \%$ yield, ${ }^{1} \mathrm{H}$ NMR (400 MHz, DMSO) $\delta 10.45$ (s, 1H), $7.80-7.77(\mathrm{~m}, 2 \mathrm{H}), 7.47-7.45(\mathrm{~m}, 4 \mathrm{H}), 7.22(\mathrm{dd}, J=8.1,1.4 \mathrm{~Hz}, 1 \mathrm{H}), 7.13(\mathrm{~d}, J=8.1$ $\mathrm{Hz}, 1 \mathrm{H}), 6.53(\mathrm{~d}, J=1.1 \mathrm{~Hz}, 1 \mathrm{H}), 2.28(\mathrm{~s}, 3 \mathrm{H}) .{ }^{13} \mathrm{C}$ NMR (150 MHz, DMSO) $\delta$ 167.8, 148.7, 138.1, 138.0, 135.1, 133.3, 131.2, 130.6, 129.3, 128.0, 127.8, 125.9, 123.2, 20.4. HRMS (ESI) Calculated for $\mathrm{C}_{16} \mathrm{H}_{13} \mathrm{NNaOS}[\mathrm{M}+\mathrm{Na}]^{+}$290.0610; found 290.0612 .

\section{6-Methyl-2-phenylbenzo $[b][1,4]$ thiazepin-4(5H)-one (1g)}<smiles>Cc1cccc2c1NC(=O)C=C(c1ccccc1)S2</smiles>

Yellow solid, $0.88 \mathrm{~g}, 33 \%$ yield, ${ }^{1} \mathrm{H}$ NMR $\left(400 \mathrm{MHz}, \mathrm{CDCl}_{3}\right) \delta 7.80-7.76$ (m, 2H), 7.69 (s, 1H), 7.47 (d, J=7.7 Hz, 1H), $7.41-7.40(\mathrm{~m}, 3 \mathrm{H}), 7.26-7.23(\mathrm{~m}, 1 \mathrm{H}), 7.11$ $(\mathrm{d}, J=7.6 \mathrm{~Hz}, 1 \mathrm{H}), 6.49(\mathrm{~s}, 1 \mathrm{H}), 2.39(\mathrm{~s}, 3 \mathrm{H}) .{ }^{13} \mathrm{C} \mathrm{NMR}\left(150 \mathrm{MHz}, \mathrm{CDCl}_{3}\right) \delta 168.6,152.5$, 138.1, 138.0, 131.7, 131.0, 130.8, 130.3, 129.6, 128.6, 128.0, 125.8, 123.7, 18.6. HRMS (ESI) Calculated for $\mathrm{C}_{16} \mathrm{H}_{13} \mathrm{NNaOS}[\mathrm{M}+\mathrm{Na}]^{+} 290.0610$; found 290.0613 . 
<smiles>COc1ccc2c(c1)SC(c1ccccc1)=CC(=O)N2</smiles>

Yellow solid, $1.13 \mathrm{~g}, 40 \%$ yield, ${ }^{1} \mathrm{H}$ NMR (400 MHz, DMSO) $\delta 10.37$ (s, 1H), $7.81-7.78(\mathrm{~m}, 2 \mathrm{H}), 7.46-7.44(\mathrm{~m}, 3 \mathrm{H}), 7.22(\mathrm{~d}, J=2.8 \mathrm{~Hz}, 1 \mathrm{H}), 7.17(\mathrm{~d}, J=8.8 \mathrm{~Hz}$, $1 \mathrm{H}), 7.00(\mathrm{dd}, J=8.8,2.8 \mathrm{~Hz}, 1 \mathrm{H}), 6.53(\mathrm{~s}, 1 \mathrm{H}), 3.77(\mathrm{~s}, 3 \mathrm{H}) .{ }^{13} \mathrm{C}$ NMR $(150 \mathrm{MHz}, \mathrm{DMSO}) \delta$ $167.7,156.8,148.5,138.1,133.7,130.5,129.6,129.3,127.9,126.0,124.4,117.4,116.7,56.1$.

HRMS (ESI) Calculated for $\mathrm{C}_{16} \mathrm{H}_{13} \mathrm{NNaO}_{2} \mathrm{~S}[\mathrm{M}+\mathrm{Na}]^{+}$306.0559; found 306.0555.

\section{2-(4-Fluorophenyl)benzo[b][1,4]thiazepin-4(5H)-one (1i)}<smiles>O=C1C=C(c2ccc(F)cc2)Sc2ccccc2N1</smiles>

Yellow solid, $1.33 \mathrm{~g}, 49 \%$ yield, ${ }^{1} \mathrm{H}$ NMR (400 MHz, DMSO) $\delta 10.56$ (s, 1H), $7.90-7.81(\mathrm{~m}, 2 \mathrm{H}), 7.65(\mathrm{dd}, J=7.7,1.1 \mathrm{~Hz}, 1 \mathrm{H}), 7.46-7.40(\mathrm{~m}, 1 \mathrm{H}), 7.30(\mathrm{t}, J=8.8$ $\mathrm{Hz}, 2 \mathrm{H}), 7.27-7.22(\mathrm{~m}, 1 \mathrm{H}), 7.22-7.17(\mathrm{~m}, 1 \mathrm{H}), 6.55(\mathrm{~s}, 1 \mathrm{H}) .{ }^{13} \mathrm{C}$ NMR (150 MHz, DMSO) $\delta 167.7,163.7(\mathrm{~d}, J=247.7 \mathrm{~Hz}), 147.6,140.7,134.3(\mathrm{~d}, J=3.0 \mathrm{~Hz}), 133.2,130.7,130.2(\mathrm{~d}, J$ $=8.7 \mathrm{~Hz}), 127.9,125.9,125.6,123.4,116.3(\mathrm{~d}, J=21.9 \mathrm{~Hz})$. HRMS (ESI) Calculated for $\mathrm{C}_{15} \mathrm{H}_{10} \mathrm{FNNaOS}[\mathrm{M}+\mathrm{Na}]^{+}$294.0359; found 294.0353.

\section{2-(4-Chlorophenyl)benzo[b][1,4]thiazepin-4(5H)-one $(1 \mathrm{j})$}<smiles>O=C1C=C(c2ccc(Cl)cc2)Sc2ccccc2N1</smiles>

Yellow solid, $1.21 \mathrm{~g}, 42 \%$ yield, ${ }^{1} \mathrm{H}$ NMR (400 MHz, DMSO) $\delta 10.59$ (s, $1 \mathrm{H}), 7.82(\mathrm{~d}, J=8.6 \mathrm{~Hz}, 2 \mathrm{H}), 7.64(\mathrm{dd}, J=7.7,1.1 \mathrm{~Hz}, 1 \mathrm{H}), 7.53(\mathrm{~d}, J=8.6 \mathrm{~Hz}, 2 \mathrm{H}), 7.43(\mathrm{td}$, $J=8.0,1.3 \mathrm{~Hz}, 1 \mathrm{H}), 7.27-7.24(\mathrm{~m}, 1 \mathrm{H}), 7.20(\mathrm{td}, J=7.6,1.2 \mathrm{~Hz}, 1 \mathrm{H}), 6.60(\mathrm{~s}, 1 \mathrm{H}) .{ }^{13} \mathrm{C} \mathrm{NMR}$ (150 MHz, DMSO) $\delta 167.6,147.3,140.7,136.7,135.3,133.2,130.8,129.6,129.3,127.9,126.5$, 125.7, 123.4. HRMS (ESI) Calculated for $\mathrm{C}_{15} \mathrm{H}_{10} \mathrm{ClNNaOS}[\mathrm{M}+\mathrm{Na}]^{+}$310.0064; found 
2-(4-(Trifluoromethyl)phenyl)benzo[b][1,4]thiazepin-4(5H)-one (1k)<smiles>O=C1C=C(c2ccc(C(F)(F)F)cc2)Sc2ccccc2N1</smiles>

Yellow solid, $1.25 \mathrm{~g}, 39 \%$ yield, ${ }^{1} \mathrm{H}$ NMR $\left(400 \mathrm{MHz}, \mathrm{CDCl}_{3}\right) \delta 8.60(\mathrm{~s}$, $1 \mathrm{H}), 7.88(\mathrm{~d}, J=8.1 \mathrm{~Hz}, 2 \mathrm{H}), 7.67(\mathrm{~d}, J=8.3 \mathrm{~Hz}, 2 \mathrm{H}), 7.60(\mathrm{dd}, J=7.8,1.3 \mathrm{~Hz}, 1 \mathrm{H}), 7.40(\mathrm{td}$, $J=7.7,1.4 \mathrm{~Hz}, 1 \mathrm{H}), 7.22(\mathrm{td}, J=7.6,1.3 \mathrm{~Hz}, 1 \mathrm{H}), 7.18(\mathrm{dd}, J=7.9,1.1 \mathrm{~Hz}, 1 \mathrm{H}), 6.54(\mathrm{~d}, J=$ $1.7 \mathrm{~Hz}, 1 \mathrm{H}) .{ }^{13} \mathrm{C} \mathrm{NMR}\left(150 \mathrm{MHz}, \mathrm{CDCl}_{3}\right) \delta 168.8,150.7,141.7,139.5,132.9,132.0(\mathrm{q}, J=$ $32.2 \mathrm{~Hz}), 130.44,128.3,128.2,126.0,125.7,125.7$ (q, $J=3.7 \mathrm{~Hz}), 123.8$ (q, $J=271.7 \mathrm{~Hz})$, $122.8(\mathrm{q}, J=16.9 \mathrm{~Hz})$. HRMS (ESI) Calculated for $\mathrm{C}_{16} \mathrm{H}_{10} \mathrm{~F}_{3} \mathrm{NNaOS}[\mathrm{M}+\mathrm{Na}]^{+}$344.0327; found 344.0321 .

Methyl 4-(4-oxo-4,5-dihydrobenzo[b][1,4]thiazepin-2-yl)benzoate (11)<smiles>CC(=O)c1ccc(C2=CC(=O)Nc3ccccc3S2)cc1</smiles>

Yellow solid, $1.28 \mathrm{~g}, 41 \%$ yield, ${ }^{1} \mathrm{H}$ NMR (400 MHz, DMSO) $\delta$ $10.65(\mathrm{~s}, 1 \mathrm{H}), 8.00-7.93(\mathrm{~m}, 4 \mathrm{H}), 7.65(\mathrm{~s}, 1 \mathrm{H}), 7.43(\mathrm{~s}, 1 \mathrm{H}), 7.26-7.19(\mathrm{~m}, 2 \mathrm{H}), 6.68(\mathrm{~s}, 1 \mathrm{H})$, 3.86 (s, 3H). ${ }^{13} \mathrm{C}$ NMR (100 MHz, DMSO) $\delta$ 167.4, 166.1, 147.3, 142.3, 140.6, 133.2, 131.1, $130.8,130.0,128.2,128.0,127.9,125.7,123.4,52.8$. HRMS (ESI) Calculated for $\mathrm{C}_{17} \mathrm{H}_{13} \mathrm{NNaO}_{3} \mathrm{~S}[\mathrm{M}+\mathrm{Na}]^{+}$334.0508; found 334.0502.

2-(4-Methoxyphenyl)benzo[b][1,4] thiazepin-4(5H)-one (1m) 
<smiles>COc1ccc(C2=CC(=O)Nc3ccccc3S2)cc1</smiles>

Yellow solid, $0.99 \mathrm{~g}, 35 \%$ yield, ${ }^{1} \mathrm{H}$ NMR (400 MHz, DMSO) $\delta 10.44$ (s, 1H), $7.76(\mathrm{~d}, J=8.8 \mathrm{~Hz}, 2 \mathrm{H}), 7.62(\mathrm{dd}, J=7.7,1.0 \mathrm{~Hz}, 1 \mathrm{H}), 7.43-7.39(\mathrm{~m}, 1 \mathrm{H}), 7.23-$ $7.21(\mathrm{~m}, 1 \mathrm{H}), 7.20-7.16(\mathrm{~m}, 1 \mathrm{H}) ., 7.01$ (d, $J=8.9 \mathrm{~Hz}, 2 \mathrm{H}), 6.45$ (d, $J=1.1 \mathrm{~Hz}, 1 \mathrm{H}), 3.80(\mathrm{~s}$, 3H); ${ }^{13} \mathrm{C}$ NMR (100 MHz, DMSO) $\delta 168.1,161.4,148.7,140.9,133.1,130.6,129.5,125.5$, 123.5, 123.2, 114.7, 55.9 ppm. HRMS (ESI) Calculated for $\mathrm{C}_{16} \mathrm{H}_{13} \mathrm{NNaO}_{2} \mathrm{~S}[\mathrm{M}+\mathrm{Na}]^{+} 306.0559$; found 306.0557 .

7-Chloro-2-(4-methoxyphenyl)benzo[b][1,4] thiazepin-4(5H)-one (1n)<smiles>COc1ccc(C2=CC(=O)Nc3cc(Cl)ccc3S2)cc1</smiles>

Yellow solid, $0.99 \mathrm{~g}, 31 \%$ yield, ${ }^{1} \mathrm{H}$ NMR (400 MHz, DMSO) $\delta$ $11.03(\mathrm{~s}, 1 \mathrm{H}), 7.76(\mathrm{~s}, 1 \mathrm{H}), 7.66(\mathrm{~d}, J=7.3 \mathrm{~Hz}, 2 \mathrm{H}), 7.35$ (dd, $J=8.2,1.3 \mathrm{~Hz}, 1 \mathrm{H}), 7.08-7.05$ (m, 4H), $3.82(\mathrm{~s}, 3 \mathrm{H}) .{ }^{13} \mathrm{C}$ NMR (150 MHz, DMSO) $\delta 160.2,159.4,136.2,132.4,131.8,131.4$, $127.3,127.2,123.2,116.9,116.4,114.8,114.7,55.8$. HRMS (ESI) Calculated for $\mathrm{C}_{16} \mathrm{H}_{12} \mathrm{ClNNaO}_{2} \mathrm{~S}[\mathrm{M}+\mathrm{Na}]^{+}$340.0169; found 340.0165.

\section{2-Methylbenzo[b][1,4] thiazepin-4(5H)-one (10)}<smiles>CC1=CC(=O)Nc2ccccc2S1</smiles>

Yellow solid, $0.65 \mathrm{~g}, 34 \%$ yield, ${ }^{1} \mathrm{H}$ NMR (400 MHz, DMSO) $\delta 10.24(\mathrm{~s}, 1 \mathrm{H})$, $7.40-7.34(\mathrm{~m}, 2 \mathrm{H}), 7.17(\mathrm{~d}, J=7.1 \mathrm{~Hz}, 1 \mathrm{H}), 7.13(\mathrm{td}, J=7.6,1.1 \mathrm{~Hz}, 1 \mathrm{H}), 6.09(\mathrm{~s}, 1 \mathrm{H}), 2.14$ (s, 3H). ${ }^{13} \mathrm{C}$ NMR (150 MHz, DMSO) $\delta 167.4,149.2,140.2,132.5,130.2,127.8,126.6,125.4$, 123.2, 27.0. 


\section{General procedure for asymmetric hydrogenation.}

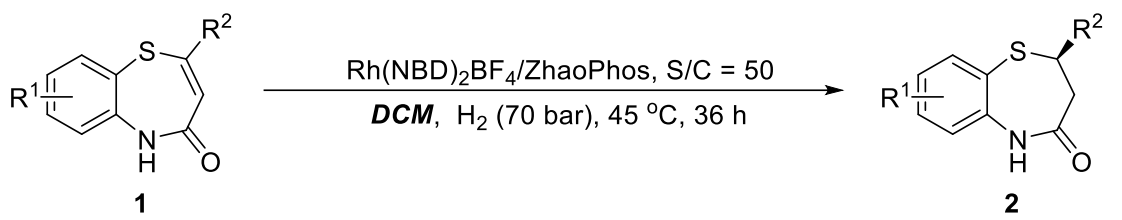

General procedure (at $\mathbf{S} / \mathbf{C}=\mathbf{5 0}$ ): To a $4.0 \mathrm{~mL}$ vial was added the catalyst precursor $\mathrm{Rh}(\mathrm{NBD}){ }_{2} \mathrm{BF}_{4}(7.48 \mathrm{mg}, 0.02 \mathrm{mmol})$, ZhaoPhos $(19.1 \mathrm{mg}, 0.022 \mathrm{mmol})$ and anhydrous DCM $(1.0 \mathrm{~mL})$ in the argon-filled glovebox. The mixture was stirred for $0.5 \mathrm{~h}$ at $25{ }^{\circ} \mathrm{C}$. An aliquot $(100 \mu \mathrm{L})$ of this complex and $0.4 \mathrm{~mL}$ anhydrous DCM were successively transferred into a 5 $\mathrm{mL}$ score-break ampule which was charged with $0.10 \mathrm{mmol}$ substrate. This ampule was placed in an autoclave. The autoclave was purged with hydrogen gas for three times, then pressurized to 70 bar $\mathrm{H}_{2}$. The reaction solution was stirred at $45^{\circ} \mathrm{C}$ (the autoclave was placed in an oil bath) for $36 \mathrm{~h}$, after which the hydrogen gas was released carefully in a fume hood. The solution of reaction mixture was purified by a flash chromatography on silica $\left(\mathrm{CH}_{2} \mathrm{Cl}_{2} / \mathrm{MeOH}, \mathrm{v} / \mathrm{v}=10 / 1\right)$. The ee values of all compounds were determined by HPLC analysis on a chiral stationary phase.

The absolute configuration of products $\mathbf{2 a}^{3}, \mathbf{2} \mathbf{b}^{5}, \mathbf{2} \mathbf{e}^{6}, \mathbf{2 i}^{7}, \mathbf{2} \mathbf{j}^{7,8}, \mathbf{2} \mathbf{k}^{7,8}, \mathbf{2} \mathbf{m}^{7,8}, \mathbf{2} \mathbf{o}^{7,8}$ were determined by comparison of analytical data with the literature ${ }^{3,5-8}$ (optical rotation data). The absolute configuration of other compounds were assigned by analogy.

\section{(R)-2-Phenyl-2,3-dihydrobenzo $[b][1,4]$ thiazepin-4(5H)-one (2a)}<smiles>O=C1CC(c2ccccc2)Sc2ccccc2N1</smiles>

White solid, flash chromatography on silica $\left(\mathrm{CH}_{2} \mathrm{Cl}_{2} / \mathrm{MeOH}, \mathrm{v} / \mathrm{v}=10 / 1\right), 24.3$ mg, 95\% yield, $99 \%$ ee. $[\alpha]_{\mathrm{D}}^{25}=-418.6\left(\mathrm{c}=0.37\right.$ in $\left.\mathrm{CHCl}_{3}\right)$. The enantiomeric excess was determined by HPLC on Chiralcel AD-3 column, $254 \mathrm{~nm}, 25^{\circ} \mathrm{C}$, $n$-hexane: $i$-PrOH $=80: 20$; flow rate $1.0 \mathrm{~mL} / \mathrm{min} ; \mathrm{t}_{\mathrm{R}}($ minor $)=7.4 \mathrm{~min}, \mathrm{t}_{\mathrm{R}}($ major $)=8.5 \mathrm{~min} .{ }^{1} \mathrm{H} \mathrm{NMR}\left(400 \mathrm{MHz}, \mathrm{CDCl}_{3}\right)$ $\delta 8.82(\mathrm{~s}, 1 \mathrm{H}), 7.67(\mathrm{dd}, J=7.6,1.1 \mathrm{~Hz}, 1 \mathrm{H}), 7.43(\mathrm{td}, J=7.7,1.4 \mathrm{~Hz}, 1 \mathrm{H}), 7.34-7.21(\mathrm{~m}$, $7 \mathrm{H}), 4.89(\mathrm{dd}, J=11.3,5.6 \mathrm{~Hz}, 1 \mathrm{H}), 2.94-2.88(\mathrm{~m}, 1 \mathrm{H}), 2.82(\mathrm{ddd}, J=12.6,5.6,0.9 \mathrm{~Hz}, 1 \mathrm{H})$; ${ }^{13} \mathrm{C}$ NMR $\left(100 \mathrm{MHz}, \mathrm{CDCl}_{3}\right) \delta 172.7,143.5,141.4,135.9,130.2,128.8,127.9,126.7,126.5$, 
126.4, 123.3, 53.3, 41.6 ppm. HRMS (ESI) Calculated for $\mathrm{C}_{15} \mathrm{H}_{13} \mathrm{NNaOS}[\mathrm{M}+\mathrm{Na}]^{+}$278.0610; found 278.0613 .

(R)-5-Methyl-2-phenyl-2,3-dihydrobenzo $[b][1,4]$ thiazepin-4(5H)-one (2b)<smiles>CN1C(=O)CC(c2ccccc2)Sc2ccccc21</smiles>

White solid, flash chromatography on silica $\left(\mathrm{CH}_{2} \mathrm{Cl}_{2} / \mathrm{MeOH}, \mathrm{v} / \mathrm{v}=10 / 1\right), 24.5$ $\mathrm{mg}, 91 \%$ yield, $>99 \%$ ee. $[\alpha]_{\mathrm{D}}^{25}=-478.2\left(\mathrm{c}=0.51\right.$ in $\left.\mathrm{CHCl}_{3}\right)$. The enantiomeric excess was determined by HPLC 1290 on Chiralcel AD-3 column, $254 \mathrm{~nm}, 25^{\circ} \mathrm{C}$, $n$-hexane: $i$-PrOH $=98: 2$; flow rate $0.3 \mathrm{~mL} / \mathrm{min} ; \mathrm{t}_{\mathrm{R}}($ minor $)=20.2 \mathrm{~min}, \mathrm{t}_{\mathrm{R}}($ major $)=22.0 \mathrm{~min} .{ }^{1} \mathrm{H} \mathrm{NMR}\left(400 \mathrm{MHz}, \mathrm{CDCl}_{3}\right)$ $\delta 7.63(\mathrm{~d}, J=7.5 \mathrm{~Hz}, 1 \mathrm{H}), 7.49(\operatorname{td}, J=8.0,1.3 \mathrm{~Hz}, 1 \mathrm{H}), 7.36-7.15(\mathrm{~m}, 7 \mathrm{H}), 4.81(\mathrm{dd}, J=$ 12.5, $5.0 \mathrm{~Hz}, 2 \mathrm{H}), 3.42(\mathrm{~s}, 3 \mathrm{H}), 2.87-2.75(\mathrm{~m}, 2 \mathrm{H}) ;{ }^{13} \mathrm{C} \mathrm{NMR}\left(100 \mathrm{MHz}, \mathrm{CDCl}_{3}\right) \delta 170.7$, $147.5,143.9,136.3,130.4,128.8,127.7,127.1,126.9,126.1,124.1,53.0,42.0,36.0$ ppm. HRMS (ESI) Calculated for $\mathrm{C}_{16} \mathrm{H}_{15} \mathrm{NNaOS}[\mathrm{M}+\mathrm{Na}]^{+} 292.0767$; found 292.0764.

(R)-5-Benzyl-2-phenyl-2,3-dihydrobenzo $[b][1,4]$ thiazepin-4(5H)-one $(2 \mathrm{c})$<smiles>O=C1CC(c2ccccc2)Sc2ccccc2N1Cc1ccccc1</smiles>

White solid, flash chromatography on silica $\left(\mathrm{CH}_{2} \mathrm{Cl}_{2} / \mathrm{MeOH}, \mathrm{v} / \mathrm{v}=10 / 1\right), 33.5$ $\mathrm{mg}, 97 \%$ yield, $98 \%$ ee. $[\alpha]_{\mathrm{D}}{ }^{25}=-453.1\left(\mathrm{c}=0.55\right.$ in $\left.\mathrm{CHCl}_{3}\right)$. The enantiomeric excess was determined by HPLC 1290 on Chiralcel AD-3 column, $254 \mathrm{~nm}, 28^{\circ} \mathrm{C}, n$-hexane: $i$-PrOH $=$ 80:20; flow rate $0.3 \mathrm{~mL} / \mathrm{min} ; \mathrm{t}_{\mathrm{R}}($ minor $)=6.7 \mathrm{~min}, \mathrm{t}_{\mathrm{R}}$ (major) $=9.3 \mathrm{~min} .{ }^{1} \mathrm{H} \mathrm{NMR}(400 \mathrm{MHz}$, $\left.\mathrm{CDCl}_{3}\right) \delta 7.68-7.56(\mathrm{~m}, 1 \mathrm{H}), 7.41-7.18(\mathrm{~m}, 11 \mathrm{H}), 7.14(\mathrm{~d}, J=6.8 \mathrm{~Hz}, 2 \mathrm{H}), 5.23(\mathrm{~d}, J=15.3$ $\mathrm{Hz}, 1 \mathrm{H}), 5.01(\mathrm{~d}, J=15.2 \mathrm{~Hz}, 1 \mathrm{H}), 4.85(\mathrm{dd}, J=12.6,5.6 \mathrm{~Hz}, 1 \mathrm{H}), 2.94-2.82(\mathrm{~m}, 2 \mathrm{H}) ;{ }^{13} \mathrm{C}$ $\operatorname{NMR}\left(100 \mathrm{MHz}, \mathrm{CDCl}_{3}\right) \delta 170.7,146.2,144.0,137.1,136.6,130.2,128.8,128.4,128.0,127.7$, 127.4, 127.3, 127.2, 126.1, 124.1, 52.9, 51.8, 42.1 ppm. HRMS (ESI) Calculated for $\mathrm{C}_{22} \mathrm{H}_{19} \mathrm{NNaOS}[\mathrm{M}+\mathrm{Na}]^{+}$368.1080; found 368.1083.

(R)-7-Fluoro-2-phenyl-2,3-dihydrobenzo $[b][1,4]$ thiazepin-4(5H)-one (2d) 
<smiles>O=C1CC(c2ccccc2)Sc2ccc(F)cc2N1</smiles>

White solid, flash chromatography on silica $\left(\mathrm{CH}_{2} \mathrm{Cl}_{2} / \mathrm{MeOH}, \mathrm{v} / \mathrm{v}=10 / 1\right)$, $26.2 \mathrm{mg}, 96 \%$ yield, $>99 \%$ ee. $[\alpha]_{\mathrm{D}}^{25}=-453.2\left(\mathrm{c}=0.51 \mathrm{in} \mathrm{CHCl}_{3}\right)$. The enantiomeric excess was determined by HPLC 1290 on Chiralcel AD-3 column, $254 \mathrm{~nm}, 28^{\circ} \mathrm{C}, n$-hexane: $i$-PrOH $=$ 80:20; flow rate $0.3 \mathrm{~mL} / \mathrm{min} ; \mathrm{t}_{\mathrm{R}}($ minor $)=5.5 \mathrm{~min}, \mathrm{t}_{\mathrm{R}}$ (major) $=5.9 \mathrm{~min} .{ }^{1} \mathrm{H} \mathrm{NMR}(400 \mathrm{MHz}$, $\left.\mathrm{CDCl}_{3}\right) \delta 9.03(\mathrm{~s}, 1 \mathrm{H}), 7.40(\mathrm{dd}, J=8.1,2.8 \mathrm{~Hz}, 1 \mathrm{H}), 7.35-7.28(\mathrm{~m}, 5 \mathrm{H}), 7.20(\mathrm{dd}, J=8.7$, $5.2 \mathrm{~Hz}, 1 \mathrm{H}), 7.15-7.10(\mathrm{~m}, 1 \mathrm{H}), 4.90(\mathrm{dd}, J=11.1,5.7 \mathrm{~Hz}, 1 \mathrm{H}), 2.93-2.87(\mathrm{~m}, 1 \mathrm{H}), 2.82(\mathrm{dd}$, $J=12.6,5.3 \mathrm{~Hz}, 1 \mathrm{H}) ;{ }^{13} \mathrm{C} \mathrm{NMR}\left(100 \mathrm{MHz}, \mathrm{CDCl}_{3}\right) \delta 172.7,160.0(\mathrm{~d}, J=247.4 \mathrm{~Hz}), 143.1$, $137.6(\mathrm{~d}, J=3.0 \mathrm{~Hz}), 128.9,128.5(\mathrm{~d}, J=8.4 \mathrm{~Hz}), 128.1,126.4,124.6(\mathrm{~d}, J=8.5 \mathrm{~Hz}), 122.2$ $(\mathrm{d}, J=22.7 \mathrm{~Hz}), 117.1(\mathrm{~d}, J=22.3 \mathrm{~Hz}), 53.4,41.4 \mathrm{ppm}$. HRMS (ESI) Calculated for $\mathrm{C}_{15} \mathrm{H}_{12} \mathrm{FNNaOS}[\mathrm{M}+\mathrm{Na}]^{+}$296.0516; found 296.0515.

(R)-7-Chloro-2-phenyl-2,3-dihydrobenzo $[b][1,4]$ thiazepin-4(5H)-one (2e)<smiles>O=C1CC(c2ccccc2)Sc2ccc(Cl)cc2N1</smiles>

White solid, flash chromatography on silica $\left(\mathrm{CH}_{2} \mathrm{Cl}_{2} / \mathrm{MeOH}, \mathrm{v} / \mathrm{v}=10 / 1\right)$, $24.6 \mathrm{mg}, 85 \%$ yield, $>99 \%$ ee. $[\alpha]_{\mathrm{D}}^{25}=-431.6\left(\mathrm{c}=0.33\right.$ in $\left.\mathrm{CHCl}_{3}\right)$. The enantiomeric excess was determined by HPLC 1290 on Chiralcel AD-3 column, $254 \mathrm{~nm}, 25^{\circ} \mathrm{C}, n$-hexane: $i$-PrOH $=95: 5 ;$ flow rate $0.4 \mathrm{~mL} / \mathrm{min} ; \mathrm{t}_{\mathrm{R}}($ minor $)=11.6 \mathrm{~min}, \mathrm{t}_{\mathrm{R}}$ (major) $=13.6 \mathrm{~min} .{ }^{1} \mathrm{H} \mathrm{NMR}(400 \mathrm{MHz}$, $\left.\mathrm{CDCl}_{3}\right) \delta 9.07(\mathrm{~s}, 1 \mathrm{H}), 7.59(\mathrm{~d}, J=8.1 \mathrm{~Hz}, 1 \mathrm{H}), 7.34-7.27(\mathrm{~m}, 5 \mathrm{H}), 7.24-7.20(\mathrm{~m}, 2 \mathrm{H}), 4.88$ $(\mathrm{dd}, J=10.9,5.8 \mathrm{~Hz}, 1 \mathrm{H}), 2.91(\mathrm{dd}, J=12.5,11.0 \mathrm{~Hz}, 1 \mathrm{H}), 2.84(\mathrm{ddd}, J=12.8,5.8,0.7 \mathrm{~Hz}$, $1 \mathrm{H}) ;{ }^{13} \mathrm{C} \mathrm{NMR}\left(100 \mathrm{MHz}, \mathrm{CDCl}_{3}\right) \delta 172.7,143.1,142.6,136.7,135.9,128.9,128.0,126.8$, 126.3, 125.0, 123.4, 53.3, 41.6 ppm. HRMS (ESI) Calculated for $\mathrm{C}_{15} \mathrm{H}_{12} \mathrm{ClNNaOS}[\mathrm{M}+\mathrm{Na}]^{+}$ 312.0220; found 312.0222.

(R)-7-Methyl-2-phenyl-2,3-dihydrobenzo[b][1,4] thiazepin-4(5H)-one (2f) 
<smiles>Cc1ccc2c(c1)NC(=O)CC(c1ccccc1)S2</smiles>

White solid, flash chromatography on silica $\left(\mathrm{CH}_{2} \mathrm{Cl}_{2} / \mathrm{MeOH}, \mathrm{v} / \mathrm{v}=10 / 1\right)$,

$25.1 \mathrm{mg}, 93 \%$ yield, $99 \%$ ee. $[\alpha]_{\mathrm{D}}{ }^{25}=-388.6\left(\mathrm{c}=0.36\right.$ in $\left.\mathrm{CHCl}_{3}\right)$. The enantiomeric excess was determined by HPLC 1290 on Chiralcel AD-3 column, $254 \mathrm{~nm}, 28^{\circ} \mathrm{C}, n$-hexane: $i$-PrOH $=$ 80:20; flow rate $0.3 \mathrm{~mL} / \mathrm{min} ; \mathrm{t}_{\mathrm{R}}($ minor $)=6.0 \mathrm{~min}, \mathrm{t}_{\mathrm{R}}($ major $)=6.7 \mathrm{~min} .{ }^{1} \mathrm{H} \mathrm{NMR}(400 \mathrm{MHz}$, $\left.\mathrm{CDCl}_{3}\right) \delta 8.80(\mathrm{~s}, 1 \mathrm{H}), 7.47(\mathrm{~d}, J=1.1 \mathrm{~Hz}, 1 \mathrm{H}), 7.32-7.20(\mathrm{~m}, 5 \mathrm{H}), 7.21(\mathrm{dd}, J=8.0,1.4 \mathrm{~Hz}$, 1H), $7.09(\mathrm{~d}, J=8.0 \mathrm{~Hz}, 1 \mathrm{H}), 4.87(\mathrm{dd}, J=11.2,5.5 \mathrm{~Hz}, 1 \mathrm{H}), 2.93-2.87(\mathrm{~m}, 1 \mathrm{H}), 2.80(\mathrm{ddd}$, $J=12.5,5.5,0.8 \mathrm{~Hz}, 1 \mathrm{H}), 2.37(\mathrm{~s}, 3 \mathrm{H}) ;{ }^{13} \mathrm{C} \mathrm{NMR}\left(100 \mathrm{MHz}, \mathrm{CDCl}_{3}\right) \delta 172.8,143.6,138.7$, 136.6, 136.0, 130.8, 128.8, 127.8, 126.5, 126.2, 123.1, 53.2, 41.6, 20.8 ppm. HRMS (ESI) Calculated for $\mathrm{C}_{16} \mathrm{H}_{15} \mathrm{NNaOS}[\mathrm{M}+\mathrm{Na}]^{+} 292.0767$; found 292.0762 .

(R)-6-Methyl-2-phenyl-2,3-dihydrobenzo $[b][1,4]$ thiazepin-4(5H)-one (2g)<smiles>Cc1cccc2c1NC(=O)CC(c1ccccc1)S2</smiles>

White solid, flash chromatography on silica $\left(\mathrm{CH}_{2} \mathrm{Cl}_{2} / \mathrm{MeOH}, \mathrm{v} / \mathrm{v}=10 / 1\right), 24.2$ mg, $90 \%$ yield, $99 \%$ ee. $[\alpha]_{\mathrm{D}}^{25}=-373.8\left(\mathrm{c}=0.31\right.$ in $\left.\mathrm{CHCl}_{3}\right)$. The enantiomeric excess was determined by HPLC on Chiralcel AD-3 column, $254 \mathrm{~nm}, 25^{\circ} \mathrm{C}$, $n$-hexane: $i$-PrOH $=80: 20$; flow rate $0.5 \mathrm{~mL} / \mathrm{min} ; \mathrm{t}_{\mathrm{R}}($ minor $)=13.4 \mathrm{~min}, \mathrm{t}_{\mathrm{R}}($ major $)=14.0 \mathrm{~min} .{ }^{1} \mathrm{H} \mathrm{NMR}\left(400 \mathrm{MHz}, \mathrm{CDCl}_{3}\right)$ $\delta 8.02(\mathrm{~s}, 1 \mathrm{H}), 7.52(\mathrm{~d}, J=6.9 \mathrm{~Hz}, 1 \mathrm{H}), 7.32-7.27126(\mathrm{~m}, 6 \mathrm{H}), 7.15(\mathrm{t}, J=7.6 \mathrm{~Hz}, 1 \mathrm{H}), 4.85$ $(\mathrm{dd}, J=11.0,6.0 \mathrm{~Hz}, 1 \mathrm{H}), 2.90-2.77(\mathrm{~m}, 2 \mathrm{H}), 2.38(\mathrm{~s}, 3 \mathrm{H}) ;{ }^{13} \mathrm{C} \mathrm{NMR}\left(150 \mathrm{MHz}, \mathrm{CDCl}_{3}\right) \delta$ $172.3,143.5,140.0,133.9,131.9,131.9,128.8,127.7,126.7,126.5,126.4,53.1,41.4,18.6$ ppm. HRMS (ESI) Calculated for $\mathrm{C}_{16} \mathrm{H}_{15} \mathrm{NNaOS}[\mathrm{M}+\mathrm{Na}]^{+}$292.0767; found 292.0762.

(R)-8-Methoxy-2-phenyl-2,3-dihydrobenzo[b][1,4]thiazepin-4(5H)-one (2h)<smiles>COc1ccc2c(c1)SC(c1ccccc1)CC(=O)N2</smiles>

White solid, flash chromatography on silica $\left(\mathrm{CH}_{2} \mathrm{Cl}_{2} / \mathrm{MeOH}, \mathrm{v} / \mathrm{v}=10 / 1\right)$,

$23.1 \mathrm{mg}, 81 \%$ yield, $98 \%$ ee. $[\alpha]_{\mathrm{D}}^{25}=-438.1\left(\mathrm{c}=0.56\right.$ in $\left.\mathrm{CHCl}_{3}\right)$. The enantiomeric excess was 
determined by HPLC 1290 on Chiralcel AD-3 column, $254 \mathrm{~nm}, 28^{\circ} \mathrm{C}, n$-hexane: $i$-PrOH $=$ 80:20; flow rate $0.3 \mathrm{~mL} / \mathrm{min} ; \mathrm{t}_{\mathrm{R}}($ minor $)=7.3 \mathrm{~min}, \mathrm{t}_{\mathrm{R}}$ (major) $=9.0 \mathrm{~min} .{ }^{1} \mathrm{H} \mathrm{NMR}(400 \mathrm{MHz}$, $\left.\mathrm{CDCl}_{3}\right) \delta 8.31(\mathrm{~s}, 1 \mathrm{H}), 7.32-7.28(\mathrm{~m}, 5 \mathrm{H}), 7.19(\mathrm{~d}, J=2.9 \mathrm{~Hz}, 1 \mathrm{H}), 7.12(\mathrm{~d}, J=8.7 \mathrm{~Hz}, 1 \mathrm{H})$, $6.95(\mathrm{dd}, J=8.7,2.9 \mathrm{~Hz}, 1 \mathrm{H}), 4.87$ (dd, $J=11.2,5.6 \mathrm{~Hz}, 1 \mathrm{H}), 3.83$ (s, 3H), 2.90 (dd, $J=12.3$, $11.4 \mathrm{~Hz}, 1 \mathrm{H}), 2.79(\mathrm{ddd}, J=12.5,5.6,1.0 \mathrm{~Hz}, 1 \mathrm{H}) ;{ }^{13} \mathrm{C} \mathrm{NMR}\left(150 \mathrm{MHz}, \mathrm{CDCl}_{3}\right) \delta 172.5,157.7$, $143.5,134.1,128.8,127.9,127.9,126.4,124.4,120.2,115.9,55.8,53.1,41.3$ ppm. HRMS (ESI) Calculated for $\mathrm{C}_{16} \mathrm{H}_{15} \mathrm{NNaO}_{2} \mathrm{~S}[\mathrm{M}+\mathrm{Na}]^{+}$308.0716; found 308.0711.

\section{(R)-2-(4-Fluorophenyl)-2,3-dihydrobenzo[b][1,4]thiazepin-4(5H)-one (2i)}<smiles>O=C1CC(c2ccc(F)cc2)Sc2ccccc2N1</smiles>

White solid, flash chromatography on silica $\left(\mathrm{CH}_{2} \mathrm{Cl}_{2} / \mathrm{MeOH}, \mathrm{v} / \mathrm{v}=10 / 1\right)$, $22.4 \mathrm{mg}, 82 \%$ yield, $98 \%$ ee. $[\alpha]_{\mathrm{D}}^{25}=-227.6\left(\mathrm{c}=0.52\right.$ in $\left.\mathrm{CHCl}_{3}\right)$. The enantiomeric excess was determined by HPLC 1290 on Chiralcel AD-3 column, $254 \mathrm{~nm}, 28^{\circ} \mathrm{C}, n$-hexane: $i$-PrOH $=$ 80:20; flow rate $0.3 \mathrm{~mL} / \mathrm{min} ; \mathrm{t}_{\mathrm{R}}($ minor $)=6.4 \mathrm{~min}, \mathrm{t}_{\mathrm{R}}$ (major) $=7.2 \mathrm{~min} .{ }^{1} \mathrm{H} \mathrm{NMR}(400 \mathrm{MHz}$, $\left.\mathrm{CDCl}_{3}\right) \delta 8.45(\mathrm{~s}, 1 \mathrm{H}), 7.66(\mathrm{dd}, J=7.7,1.1 \mathrm{~Hz}, 1 \mathrm{H}), 7.44(\mathrm{td}, J=7.7,1.4 \mathrm{~Hz}, 1 \mathrm{H}), 7.32-7.24$ (m, 2H), $7.25-7.19(\mathrm{~m}, 2 \mathrm{H}), 7.03-6.97(\mathrm{~m}, 2 \mathrm{H}), 4.88(\mathrm{dd}, J=10.1,6.3 \mathrm{~Hz}, 1 \mathrm{H}), 2.88-2.78$ $(\mathrm{m}, 2 \mathrm{H}) ;{ }^{13} \mathrm{C}$ NMR $\left(100 \mathrm{MHz}, \mathrm{CDCl}_{3}\right) \delta 172.2,162.2(\mathrm{~d}, J=246.0 \mathrm{~Hz}), 141.3,139.1(\mathrm{~d}, J=$ $4.0 \mathrm{~Hz}), 135.8,130.3,128.2$ (d, $J=8.0 \mathrm{~Hz}), 126.8,126.4,123.3,115.7$ (d, $J=22.0 \mathrm{~Hz}), 52.5$, 41.6 ppm. HRMS (ESI) Calculated for $\mathrm{C}_{15} \mathrm{H}_{12} \mathrm{FNNaOS}[\mathrm{M}+\mathrm{Na}]^{+}$296.0516; found 296.0511.

\section{(R)-2-(4-Chlorophenyl)-2,3-dihydrobenzo[b][1,4]thiazepin-4(5H)-one (2j)}<smiles>O=C1CC(c2ccc(Cl)cc2)Sc2ccccc2N1</smiles>

White solid, flash chromatography on silica $\left(\mathrm{CH}_{2} \mathrm{Cl}_{2} / \mathrm{MeOH}, \mathrm{v} / \mathrm{v}=10 / 1\right)$, $25.8 \mathrm{mg}, 89 \%$ yield, $>99 \%$ ee. $[\alpha]_{\mathrm{D}}{ }^{25}=-372.1\left(\mathrm{c}=0.33\right.$ in $\left.\mathrm{CHCl}_{3}\right)$. The enantiomeric excess 
was determined by HPLC on Chiralcel AD-3 column, $254 \mathrm{~nm}, 25^{\circ} \mathrm{C}, n$-hexane: $i$-PrOH $=80: 20$; flow rate $0.5 \mathrm{~mL} / \mathrm{min} ; \mathrm{t}_{\mathrm{R}}($ minor $)=16.8 \mathrm{~min}, \mathrm{t}_{\mathrm{R}}($ major $)=18.0 \mathrm{~min} .{ }^{1} \mathrm{H} \mathrm{NMR}\left(400 \mathrm{MHz}, \mathrm{CDCl}_{3}\right)$ $\delta 8.84(\mathrm{~s}, 1 \mathrm{H}), 7.65-7.63(\mathrm{~m}, 1 \mathrm{H}), 7.44(\mathrm{td}, J=7.7,1.3 \mathrm{~Hz}, 1 \mathrm{H}), 7.27-7.20(\mathrm{~m}, 6 \mathrm{H}), 4.86$ $(\mathrm{dd}, J=10.4,6.1 \mathrm{~Hz}, 1 \mathrm{H}), 2.89-2.78(\mathrm{~m}, 2 \mathrm{H}) ;{ }^{13} \mathrm{C} \mathrm{NMR}\left(150 \mathrm{MHz}, \mathrm{CDCl}_{3}\right) \delta 172.4,141.8$, 141.4, 135.8, 133.6, 130.4, 129.0, 127.9, 126.8, 126.2, 123.4, 52.6, 41.4 ppm. HRMS (ESI) Calculated for $\mathrm{C}_{15} \mathrm{H}_{12} \mathrm{ClNNaOS}[\mathrm{M}+\mathrm{Na}]^{+}$312.0220; found 312.0225 .

(R)-2-(4-(Trifluoromethyl)phenyl)-2,3-dihydrobenzo[b][1,4]thiazepin-4(5H)-one (2k)<smiles>O=C1CC(c2ccc(C(F)(F)F)cc2)Sc2ccccc2N1</smiles>

White solid, flash chromatography on silica $\left(\mathrm{CH}_{2} \mathrm{Cl}_{2} / \mathrm{MeOH}, \mathrm{v} / \mathrm{v}=\right.$ 10/1), $26.2 \mathrm{mg}, 81 \%$ yield, $95 \%$ ee. $[\alpha]_{\mathrm{D}}^{25}=-434.2\left(\mathrm{c}=0.32\right.$ in $\left.\mathrm{CHCl}_{3}\right)$. The enantiomeric excess was determined by HPLC on Chiralcel AD-3 column, $254 \mathrm{~nm}, 25^{\circ} \mathrm{C}, n$-hexane: $i$-PrOH $=80: 20$; flow rate $0.5 \mathrm{~mL} / \mathrm{min} ; \mathrm{t}_{\mathrm{R}}($ minor $)=13.7 \mathrm{~min}, \mathrm{t}_{\mathrm{R}}$ (major $)=14.3 \mathrm{~min} .{ }^{1} \mathrm{H} \mathrm{NMR}(400$ $\left.\mathrm{MHz}, \mathrm{CDCl}_{3}\right) \delta 8.74(\mathrm{~s}, 1 \mathrm{H}), 7.66(\mathrm{dd}, J=7.7,1.3 \mathrm{~Hz}, 1 \mathrm{H}), 7.58(\mathrm{~d}, J=8.2 \mathrm{~Hz}, 2 \mathrm{H}), 7.48-$ $7.43(\mathrm{~m}, 3 \mathrm{H}), 7.28-7.21(\mathrm{~m}, 2 \mathrm{H}), 4.93(\mathrm{dd}, J=10.7,5.9 \mathrm{~Hz}, 1 \mathrm{H}), 2.92-2.81(\mathrm{~m}, 2 \mathrm{H}) ;{ }^{13} \mathrm{C}$ NMR $\left(150 \mathrm{MHz}, \mathrm{CDCl}_{3}\right) \delta 172.1,147.1,141.4,135.9,130.6,130.1(\mathrm{q}, J=32.1 \mathrm{~Hz}), 127.9$, 126.9, 126.0, 125.9 (q, $J=3.5 \mathrm{~Hz}), 125.8$ (q, $J=270.2 \mathrm{~Hz}), 123.1,52.6,41.2 \mathrm{ppm}$. HRMS (ESI) Calculated for $\mathrm{C}_{16} \mathrm{H}_{12} \mathrm{~F}_{3} \mathrm{NNaOS}[\mathrm{M}+\mathrm{Na}]^{+}$346.0484; found 346.0481.

\section{Methyl (R)-4-(4-oxo-2,3,4,5-tetrahydrobenzo[b][1,4]thiazepin-2-yl)benzoate (2I)}<smiles>CC(=O)c1ccc(C2CC(=O)Nc3ccccc3S2)cc1</smiles>

White solid, flash chromatography on silica $\left(\mathrm{CH}_{2} \mathrm{Cl}_{2} / \mathrm{MeOH}\right.$, v/v $=$ 10/1), $28.8 \mathrm{mg}, 92 \%$ yield, $>99 \%$ ee. $[\alpha]_{\mathrm{D}}^{25}=-392.3\left(\mathrm{c}=0.62\right.$ in $\left.\mathrm{CHCl}_{3}\right)$. The enantiomeric excess was determined by HPLC 1290 on Chiralcel AD-3 column, $254 \mathrm{~nm}, 28^{\circ} \mathrm{C}, n$-hexane: $i$ - 
$\mathrm{PrOH}=80: 20$; flow rate $0.4 \mathrm{~mL} / \mathrm{min} ; \mathrm{t}_{\mathrm{R}}$ (major) $=7.9 \mathrm{~min}, \mathrm{t}_{\mathrm{R}}$ (minor) $=10.7 \mathrm{~min} .{ }^{1} \mathrm{H} \mathrm{NMR}$ $\left(400 \mathrm{MHz}, \mathrm{CDCl}_{3}\right) \delta 9.07(\mathrm{~s}, 1 \mathrm{H}), 7.99(\mathrm{~d}, J=8.4 \mathrm{~Hz}, 2 \mathrm{H}), 7.65-7.64(\mathrm{~m}, 1 \mathrm{H}), 7.44(\mathrm{td}, J=$ 7.7, $1.5 \mathrm{~Hz}, 1 \mathrm{H}), 7.38$ (d, $J=8.3 \mathrm{~Hz}, 2 \mathrm{H}), 7.25$ (dd, $J=12.2,4.5 \mathrm{~Hz}, 2 \mathrm{H}), 4.92$ (dd, $J=11.1$, $5.6 \mathrm{~Hz}, 1 \mathrm{H}), 3.91(\mathrm{~s}, 3 \mathrm{H}), 2.94-2.88(\mathrm{~m}, 1 \mathrm{H}), 2.82(\mathrm{dd}, J=12.5,5.7 \mathrm{~Hz}, 1 \mathrm{H}) ;{ }^{13} \mathrm{C} \mathrm{NMR}(150$ $\left.\mathrm{MHz}, \mathrm{CDCl}_{3}\right) \delta 172.4,166.6,148.2,141.5,135.9,130.5,130.2,129.6,126.8,126.5,126.0$, 123.4, 52.9, 52.2, 41.2 ppm. HRMS (ESI) Calculated for $\mathrm{C}_{17} \mathrm{H}_{15} \mathrm{NNaO}_{3} \mathrm{~S}[\mathrm{M}+\mathrm{Na}]^{+}$336.0665; found 336.0662 .

(R)-2-(4-Methoxyphenyl)-2,3-dihydrobenzo $[b][1,4]$ thiazepin-4(5H)-one (2m)<smiles>COc1ccc([C@@H]2CC(=O)Nc3ccccc3S2)cc1</smiles>

White solid, flash chromatography on silica $\left(\mathrm{CH}_{2} \mathrm{Cl}_{2} / \mathrm{MeOH}, \mathrm{v} / \mathrm{v}=\right.$ 10/1), $25.7 \mathrm{mg}, 90 \%$ yield, $98 \%$ ee. $[\alpha]_{\mathrm{D}}^{25}=-396.4\left(\mathrm{c}=0.33\right.$ in $\left.\mathrm{CHCl}_{3}\right)$. The enantiomeric excess was determined by HPLC 1290 on Chiralcel AD-3 column, $254 \mathrm{~nm}, 28^{\circ} \mathrm{C}, n$-hexane: $i$ $\operatorname{PrOH}=80: 20$; flow rate $0.3 \mathrm{~mL} / \mathrm{min} ; \mathrm{t}_{\mathrm{R}}($ minor $)=9.2 \mathrm{~min}, \mathrm{t}_{\mathrm{R}}$ (major) $=10.4 \mathrm{~min} .{ }^{1} \mathrm{H}$ NMR $\left(400 \mathrm{MHz}, \mathrm{CDCl}_{3}\right) \delta 8.54(\mathrm{~s}, 1 \mathrm{H}), 7.66-7.64(\mathrm{~m}, 1 \mathrm{H}), 7.42(\mathrm{td}, J=7.7,1.2 \mathrm{~Hz}, 1 \mathrm{H}), 7.26-$ $7.18(\mathrm{~m}, 4 \mathrm{H}), 6.84(\mathrm{~d}, J=8.7 \mathrm{~Hz}, 2 \mathrm{H}), 4.87$ (dd, $J=10.6,5.8 \mathrm{~Hz}, 1 \mathrm{H}), 3.98-3.74(\mathrm{~m}, 3 \mathrm{H})$, $2.90-2.78(\mathrm{~m}, 2 \mathrm{H}) ;{ }^{13} \mathrm{C}$ NMR $\left(101 \mathrm{MHz}, \mathrm{CDCl}_{3}\right) \delta 172.0,159.2,141.2,135.9,135.5,130.1$, 127.6, 126.9, 126.8, 123.2, 114.1, 55.3, 52.7, 41.6 ppm. HRMS (ESI) Calculated for $\mathrm{C}_{16} \mathrm{H}_{15} \mathrm{NNaO}_{2} \mathrm{~S}[\mathrm{M}+\mathrm{Na}]^{+}$308.0716; found 308.0711.

(R)-7-Chloro-2-(4-methoxyphenyl)-2,3-dihydrobenzo[b][1,4] thiazepin-4(5H)-one (2n)<smiles>COc1ccc([C@@H]2CC(=O)Nc3cc(Cl)ccc3S2)cc1</smiles>

White solid, flash chromatography on silica $\left(\mathrm{CH}_{2} \mathrm{Cl}_{2} / \mathrm{MeOH}, \mathrm{v} / \mathrm{v}\right.$ $=10 / 1), 29.4 \mathrm{mg}, 92 \%$ yield, $76 \%$ ee. $[\alpha]_{\mathrm{D}}{ }^{25}=-226.6\left(\mathrm{c}=0.35\right.$ in $\left.\mathrm{CHCl}_{3}\right)$. The enantiomeric 
excess was determined by HPLC on Chiralcel AD-3 column, $254 \mathrm{~nm}, 25^{\circ} \mathrm{C}, n$-hexane: $i$-PrOH $=80: 20$; flow rate $0.5 \mathrm{~mL} / \mathrm{min} ; \mathrm{t}_{\mathrm{R}}$ (minor) $=17.8 \mathrm{~min}, \mathrm{t}_{\mathrm{R}}$ (major) $=18.5 \mathrm{~min} .{ }^{1} \mathrm{H} \mathrm{NMR}(400$ $\left.\mathrm{MHz}, \mathrm{CDCl}_{3}\right) \delta 9.78(\mathrm{~s}, 1 \mathrm{H}), 7.22(\mathrm{~d}, J=8.3 \mathrm{~Hz}, 1 \mathrm{H}), 7.10(\mathrm{~d}, J=8.6 \mathrm{~Hz}, 2 \mathrm{H}), 7.00(\mathrm{dd}, J=$ 8.3, 2.1 Hz, 1H), $6.92(\mathrm{t}, J=2.1 \mathrm{~Hz}, 1 \mathrm{H}), 6.85-6.81(\mathrm{~m}, 2 \mathrm{H}), 3.77(\mathrm{~s}, 3 \mathrm{H}), 3.61$ (dd, $J=9.5$, $5.6 \mathrm{~Hz}, 1 \mathrm{H}), 3.21(\mathrm{dd}, J=14.1,5.6 \mathrm{~Hz}, 1 \mathrm{H}), 2.78(\mathrm{dd}, J=14.2,9.5 \mathrm{~Hz}, 1 \mathrm{H}) ;{ }^{13} \mathrm{C}$ NMR $(150$ $\left.\mathrm{MHz}, \mathrm{CDCl}_{3}\right) \delta 168.2,158.7,137.0,132.7,130.4,129.2,128.7,124.1,117.1,117.0,113.9,55.2$, 44.4, 34.9 ppm. HRMS (ESI) Calculated for $\mathrm{C}_{16} \mathrm{H}_{14} \mathrm{ClNNaO}_{2} \mathrm{~S}[\mathrm{M}+\mathrm{Na}]^{+}$342.0326; found 342.0321.

(S)-2-Methyl-2,3-dihydrobenzo[b][1,4]thiazepin-4(5H)-one (2o)<smiles>CC1CC(=O)Nc2ccccc2S1</smiles>

White solid, flash chromatography on silica $\left(\mathrm{CH}_{2} \mathrm{Cl}_{2} / \mathrm{MeOH}, \mathrm{v} / \mathrm{v}=10 / 1\right), 18.4$ $\mathrm{mg}, 95 \%$ yield, $95 \%$ ee. $[\alpha]_{\mathrm{D}}{ }^{25}=-218.6\left(\mathrm{c}=0.45\right.$ in $\left.\mathrm{CHCl}_{3}\right)$. The enantiomeric excess was determined by HPLC 1290 on Chiralcel AD-3 column, $254 \mathrm{~nm}, 28^{\circ} \mathrm{C}, n$-hexane: $i$-PrOH $=$ 80:20; flow rate $0.3 \mathrm{~mL} / \mathrm{min} ; \mathrm{t}_{\mathrm{R}}$ (major) $=5.0 \mathrm{~min}, \mathrm{t}_{\mathrm{R}}$ (minor) $=7.0 \mathrm{~min} .{ }^{1} \mathrm{H} \mathrm{NMR}(400 \mathrm{MHz}$, $\left.\mathrm{CDCl}_{3}\right) \delta 8.03(\mathrm{~s}, 1 \mathrm{H}), 7.61(\mathrm{dd}, J=7.7,1.4 \mathrm{~Hz}, 1 \mathrm{H}), 7.37$ (td, $\left.J=7.7,1.5 \mathrm{~Hz}, 1 \mathrm{H}\right), 7.17(\mathrm{td}, J$ $=7.6,1.3 \mathrm{~Hz}, 1 \mathrm{H}), 7.11(\mathrm{dd}, J=7.8,1.0 \mathrm{~Hz}, 1 \mathrm{H}), 3.90(\mathrm{dp}, J=8.5,6.6 \mathrm{~Hz}, 1 \mathrm{H}), 2.66(\mathrm{ddd}, J=$ 12.5, 5.8, $0.7 \mathrm{~Hz}, 1 \mathrm{H}), 2.35(\mathrm{dd}, J=12.5,8.7 \mathrm{~Hz}, 1 \mathrm{H}), 1.43(\mathrm{~d}, J=6.7 \mathrm{~Hz}, 3 \mathrm{H}) ;{ }^{13} \mathrm{C}$ NMR $(150$ $\left.\mathrm{MHz}, \mathrm{CDCl}_{3}\right) \delta 172.6,141.4,135.9,129.9,126.8,126.3,123.1,44.8,41.3,23.6$ ppm. HRMS (ESI) Calculated for $\mathrm{C}_{10} \mathrm{H}_{11} \mathrm{NNaOS}[\mathrm{M}+\mathrm{Na}]^{+}$216.0454; found 216.0450.

Gram-scale asymmetric hydrogenation of 2-phenylbenzo $[b][1,4]$ thiazepin-4(5H)-one (1a).
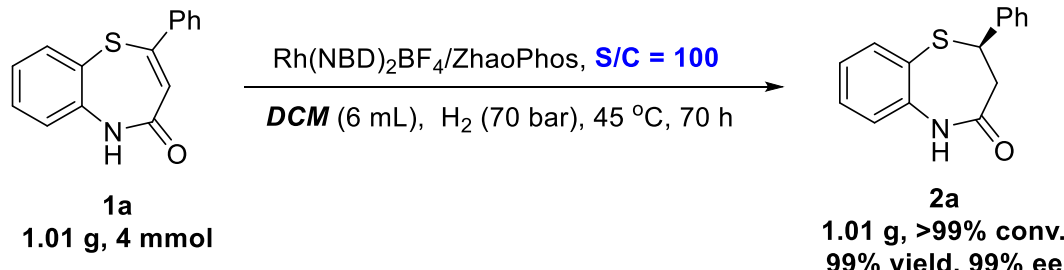

To a $4.0 \mathrm{~mL}$ vial was added the catalyst precursor $\mathrm{Rh}(\mathrm{NBD}){ }_{2} \mathrm{BF}_{4}(15.0 \mathrm{mg}, 0.04 \mathrm{mmol})$, ZhaoPhos (38.2 mg, $0.044 \mathrm{mmol})$ and anhydrous DCM (3.0 mL) in the argon-filled glovebox. 
The mixture was stirred for $0.5 \mathrm{~h}$ at $25{ }^{\circ} \mathrm{C}$. Then $4 \mathrm{mmol}$ of substrate $1 \mathrm{a}(1.01 \mathrm{~g})$ were added into a $10 \mathrm{~mL}$ hydrogenation vessel. $3 \mathrm{~mL}$ anhydrous DCM was added and a solution of $\mathrm{Rh}(\mathrm{NBD})_{2} \mathrm{BF}_{4} /$ ZhaoPhos in anhydrous DCM $(3.0 \mathrm{~mL})$ was added via an injection port. Then the vessel was placed in an autoclave, closed and moved it out from glovebox. The autoclave quickly purged with hydrogen gas for three times, then pressurized to 70 bar $\mathrm{H}_{2}$. The reaction solution was stirred at $45{ }^{\circ} \mathrm{C}$ (the autoclave was placed in an oil bath) for $70 \mathrm{~h}$, then released pressure carefully. The solution of reaction mixture was purified by a flash chromatography on silica with DCM and the solvent was removed under reduced pressure to afford the $(R)-2$ phenyl-2,3-dihydrobenzo[b][1,4]thiazepin-4(5H)-one $\mathbf{2 a} 1.01 \mathrm{~g}$ as a white solid, $>99 \%$ conversion, $99 \%$ yield, $99 \%$ ee.

\section{Synthesis of thiazesim ${ }^{8}$ :}
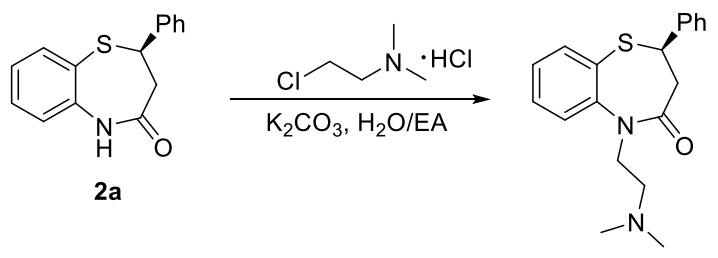

$85 \%$ yield, $>99 \%$ ee

To a solution of $(R)$-2-phenyl-2,3-dihydrobenzo[b][1,4]thiazepin-4(5H)-one $\mathbf{2 a}$ (26 mg, 0.1 mmol, 99\% ee) in EtOAc (1.0 mL) was added 2-dimethylaminoethylchloride hydrochloride (29 $\mathrm{mg}, 0.2 \mathrm{mmol})$, followed by $\mathrm{K}_{2} \mathrm{CO}_{3}(55 \mathrm{mg}, 0.4 \mathrm{mmol})$ and $\mathrm{H}_{2} \mathrm{O}(10 \mu \mathrm{L})$. After the mixture was stirred for $12 \mathrm{~h}$ under reflux in an oil bath, it was cooled to ambient temperature. The organic layers were washed with $\mathrm{H}_{2} \mathrm{O}(5 \mathrm{~mL} \times 2)$ and brine. The combined organic layers were dried over $\mathrm{Na}_{2} \mathrm{SO}_{4}$, and concentrated in vacuo. Purification by flash chromatography onsilica using $\mathrm{CH}_{2} \mathrm{Cl}_{2} / \mathrm{MeOH}(\mathrm{v} / \mathrm{v}=10 / 1)$ as an eluent gave (R)-5-(2-(dimethylamino)ethyl)-2-phenyl-2,3dihydrobenzo $[b][1,4]$ thiazepin-4(5H)-one 3 (thiazesim, $27.8 \mathrm{mg}, 85 \%$ yield, $>99 \%$ ee). colorless oil, $[\alpha]_{\mathrm{D}}{ }^{25}=-443.2\left(\mathrm{c}=1.00\right.$ in $\left.\mathrm{CHCl}_{3}\right)$. The enantiomeric excess was determined by HPLC on Chiralcel OD-H column, $220 \mathrm{~nm}, 25^{\circ} \mathrm{C}, n$-hexane: $i$-PrOH $=95: 5$; flow $1.0 \mathrm{~mL} / \mathrm{min}$; $\mathrm{t}_{\mathrm{R}}($ minor $)=12.5 \mathrm{~min}, \mathrm{t}_{\mathrm{R}}$ (major) $=15.7 \mathrm{~min} .{ }^{1} \mathrm{H} \mathrm{NMR}\left(400 \mathrm{MHz}, \mathrm{CDCl}_{3}\right) \delta 7.63-7.61(\mathrm{~m}$, 1H), $7.52-7.45(\mathrm{~m}, 2 \mathrm{H}), 7.30-7.20(\mathrm{~m}, 4 \mathrm{H}), 7.14-7.12(\mathrm{~m}, 2 \mathrm{H}), 4.79(\mathrm{dd}, J=12.5,5.6 \mathrm{~Hz}$, $1 \mathrm{H}), 4.30(\mathrm{ddd}, J=13.4,9.7,5.9 \mathrm{~Hz}, 1 \mathrm{H}), 3.70-3.63(\mathrm{~m}, 1 \mathrm{H}), 2.83-2.65(\mathrm{~m}, 3 \mathrm{H}), 2.41-$ $2.34(\mathrm{~m}, 1 \mathrm{H}), 2.24(\mathrm{~s}, 6 \mathrm{H}) .{ }^{13} \mathrm{C} \mathrm{NMR}\left(100 \mathrm{MHz}, \mathrm{CDCl}_{3}\right) \delta 170.3,146.3,143.8,136.3,130.4$, 
128.6, 127.6, 127.5, 127.1, 126.0, 124.6, 56.3, 52.7, 47.4, 45.6, $41.8 \mathrm{ppm}$. The absolute configuration of products 3 were determined by comparison of analytical data with the literature..$^{1 b, 5,9}$ 


\section{NMR Spectra}

2-phenylbenzo[ $b][1,4]$ thiazepin-4(5H)-one 1a
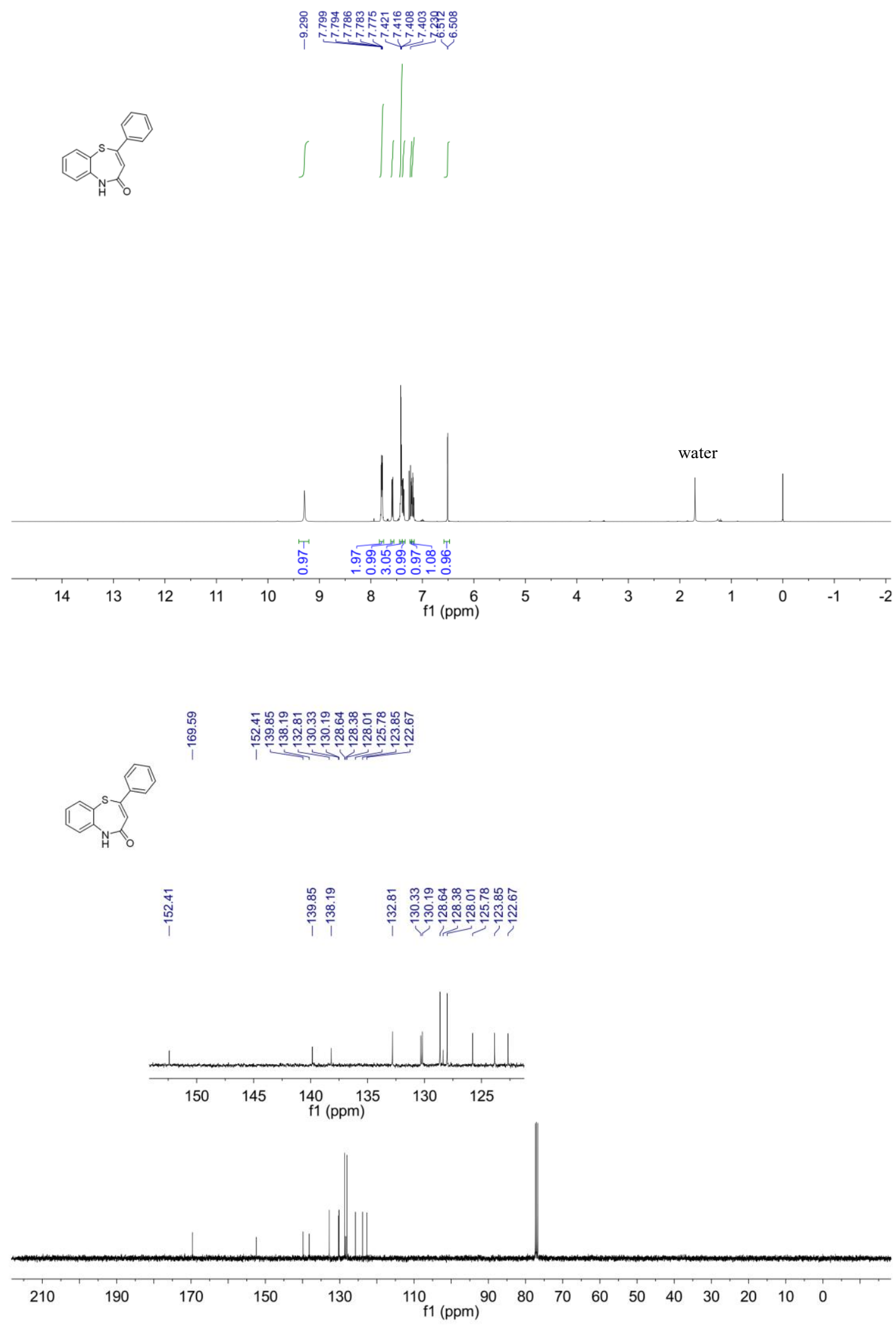
5-methyl-2-phenylbenzo[b][1,4]thiazepin-4(5H)-one 1b

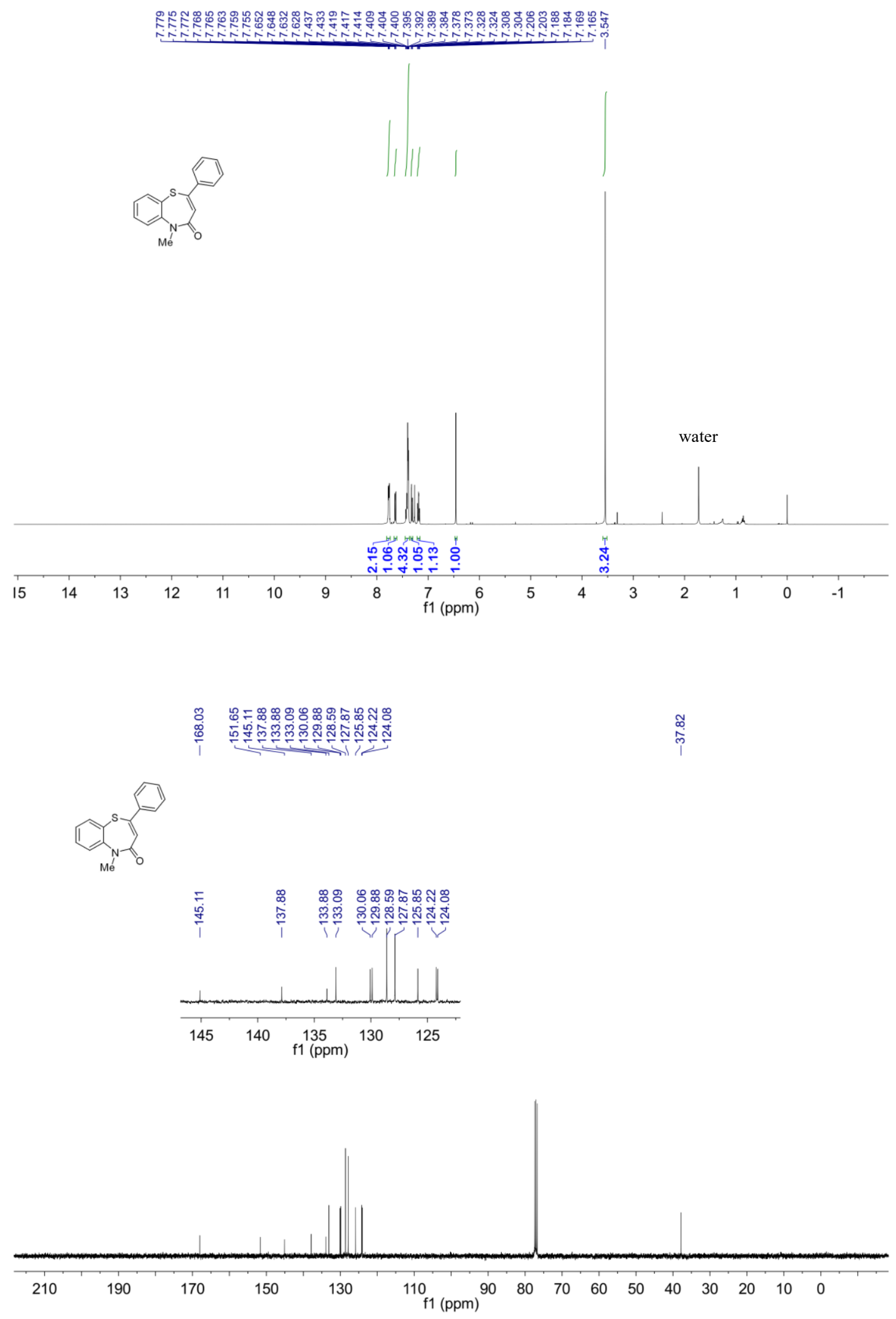


5-benzyl-2-phenylbenzo[b][1,4]thiazepin-4(5H)-one 1c
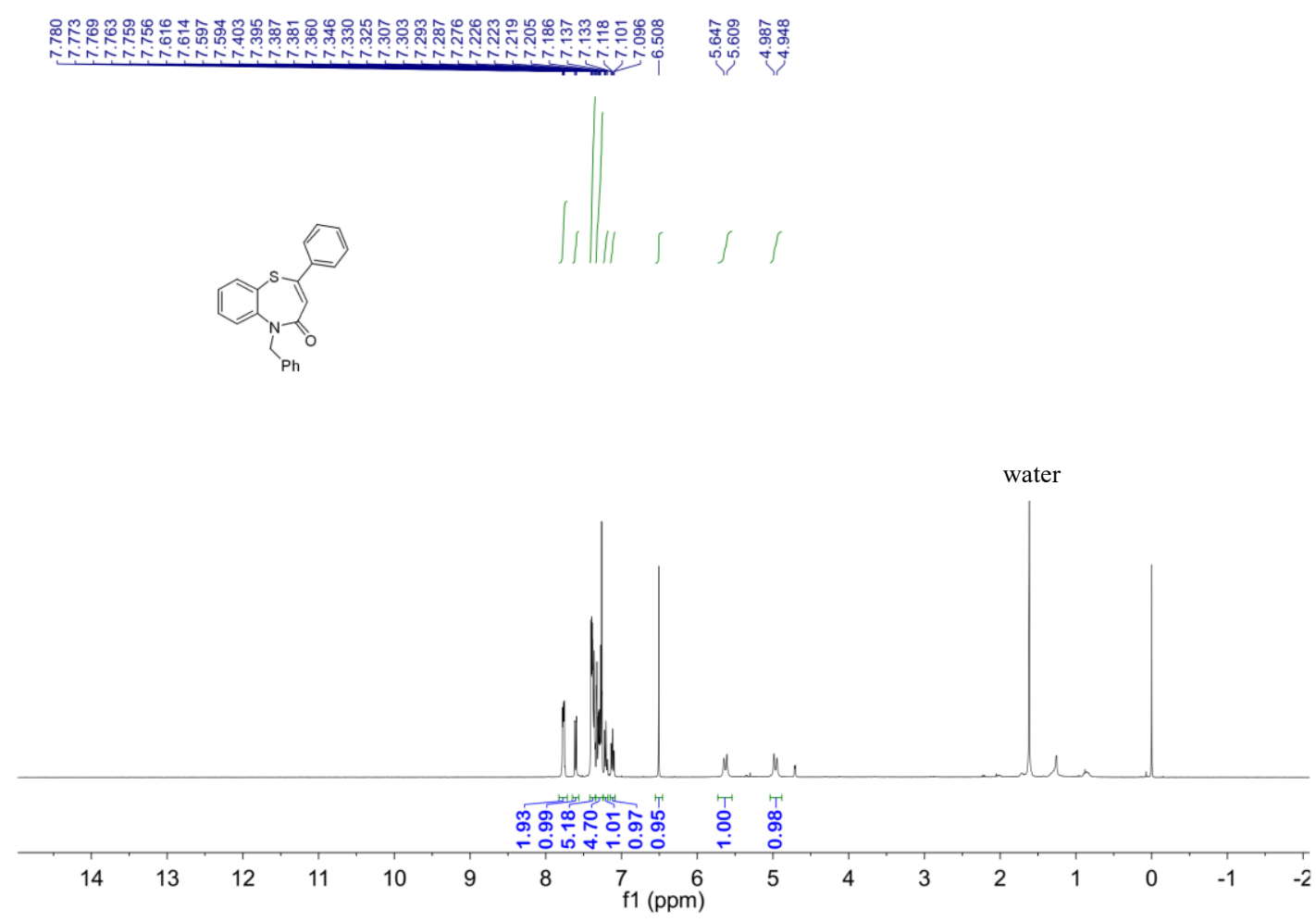

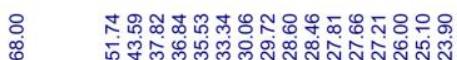

ธับํํำ

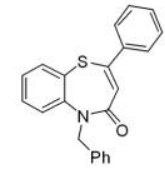

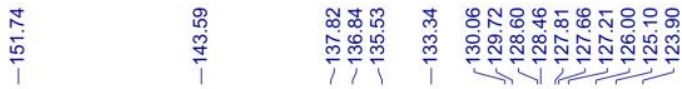
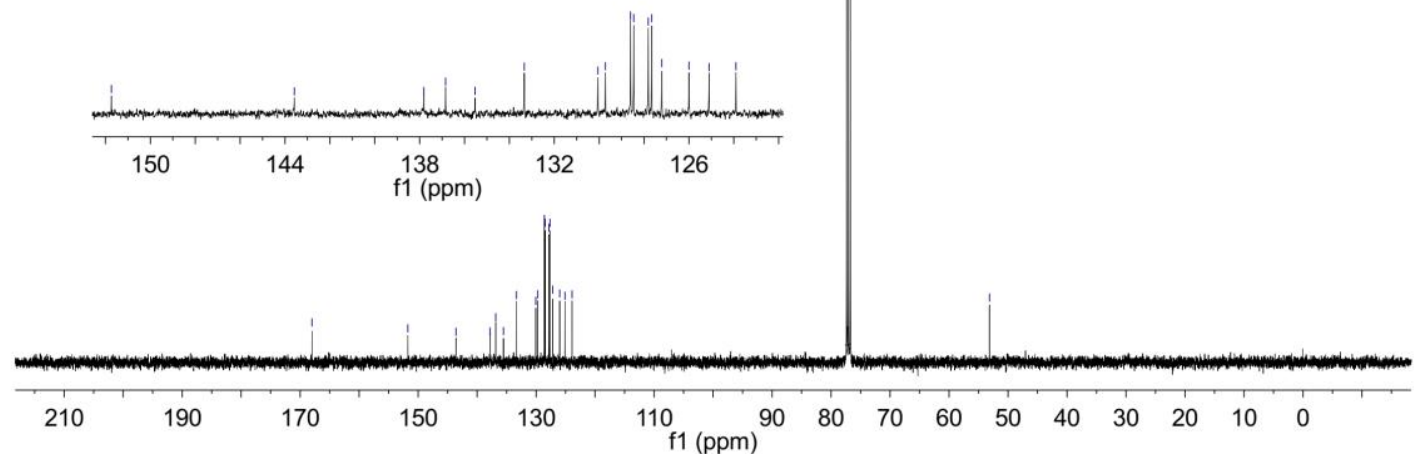
7-fluoro-2-phenylbenzo[b][1,4]thiazepin-4(5H)-one 1d
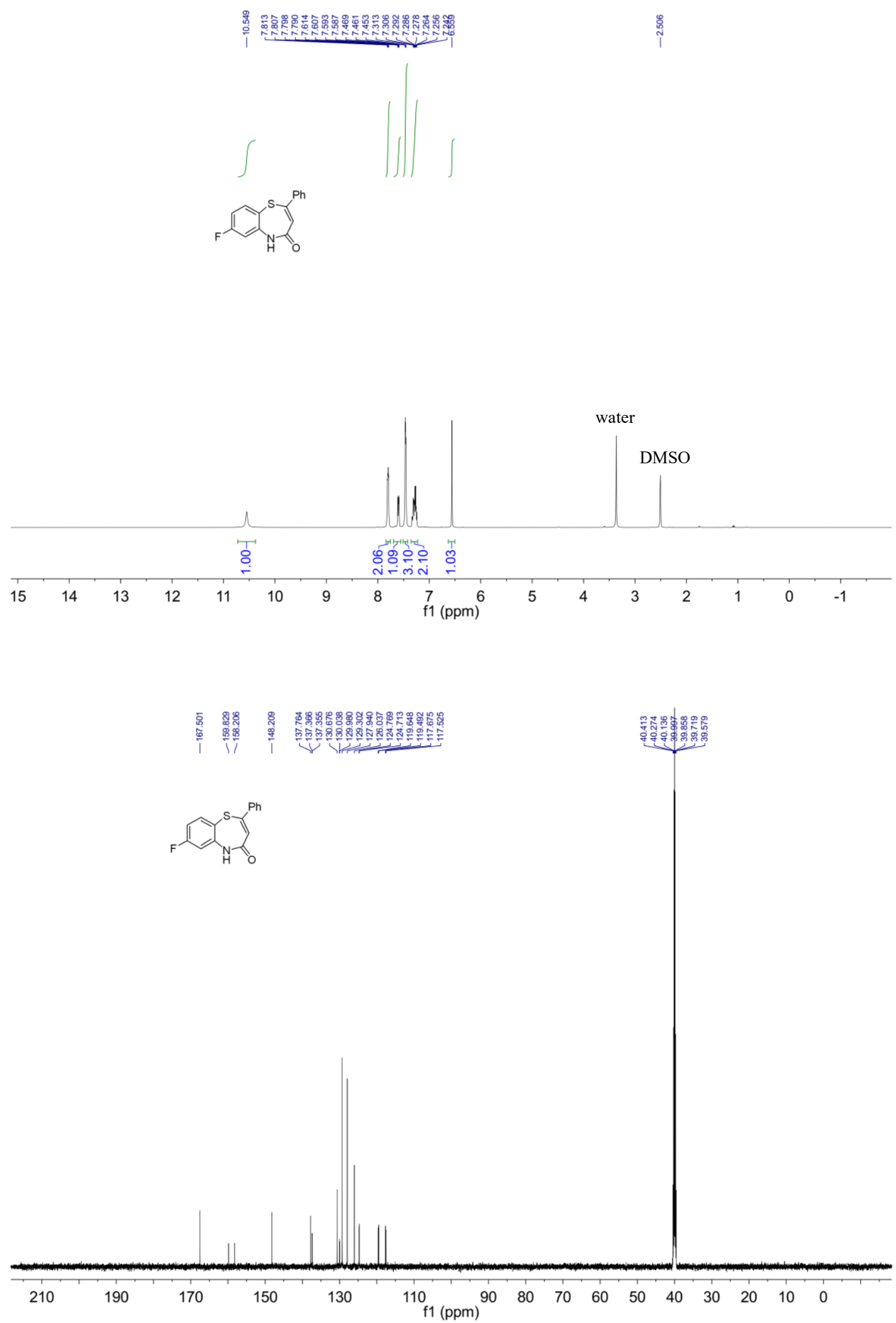
7-chloro-2-phenylbenzo[b][1,4]thiazepin-4(5H)-one 1e
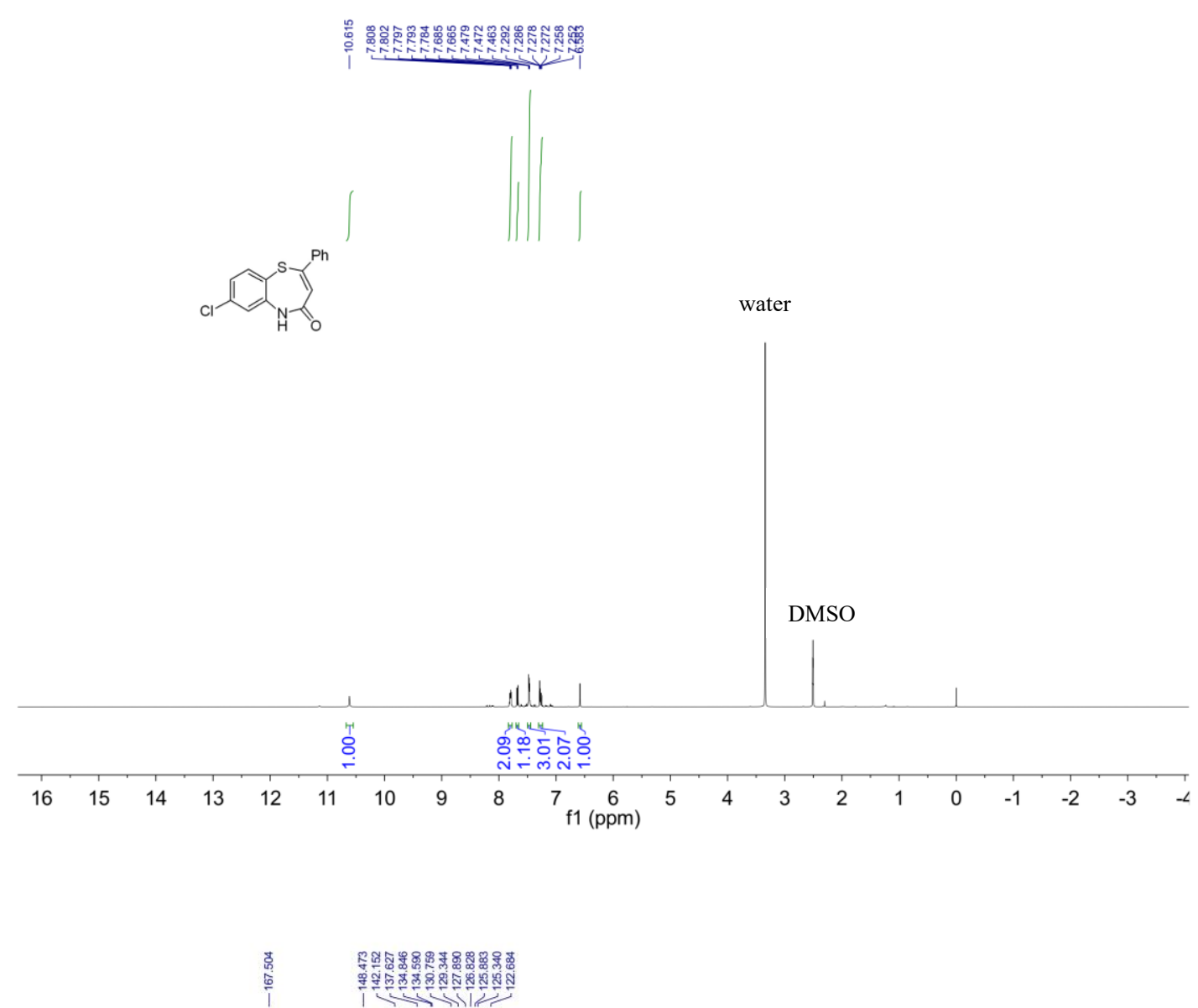

$\overbrace{\mathrm{H}}^{\mathrm{S}}$

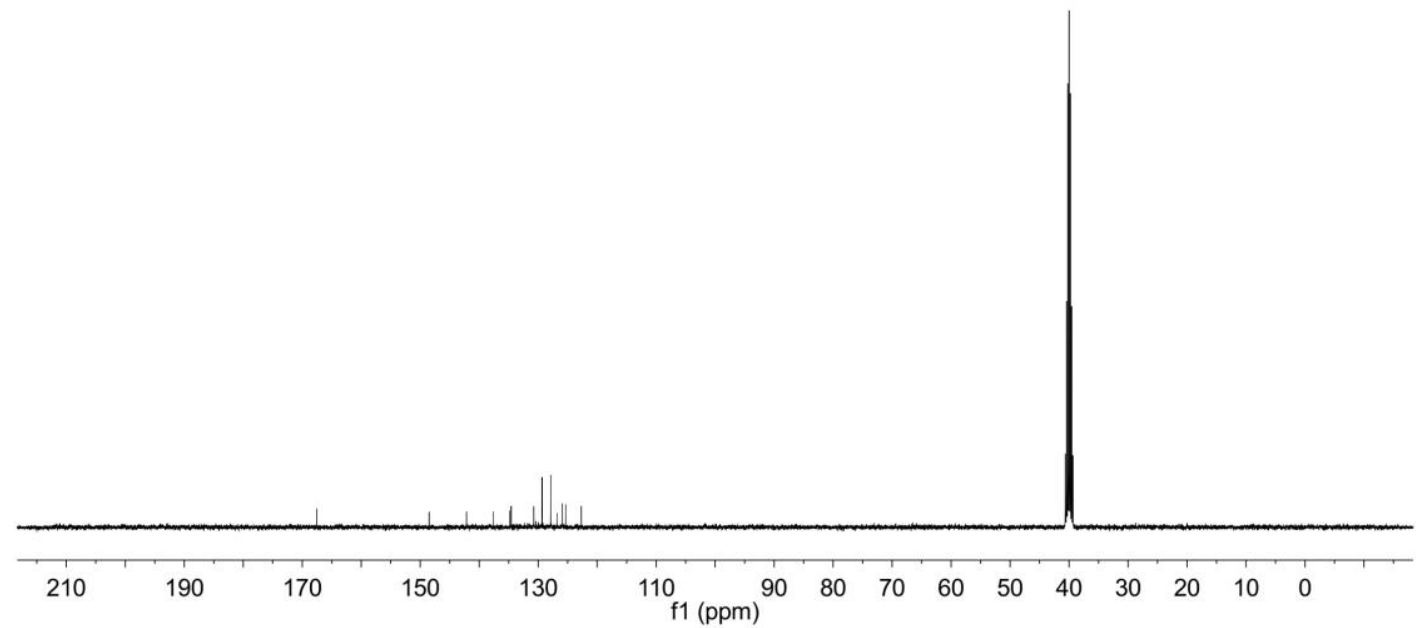


7-methyl-2-phenylbenzo[ $b][1,4]$ thiazepin-4(5H)-one 1f
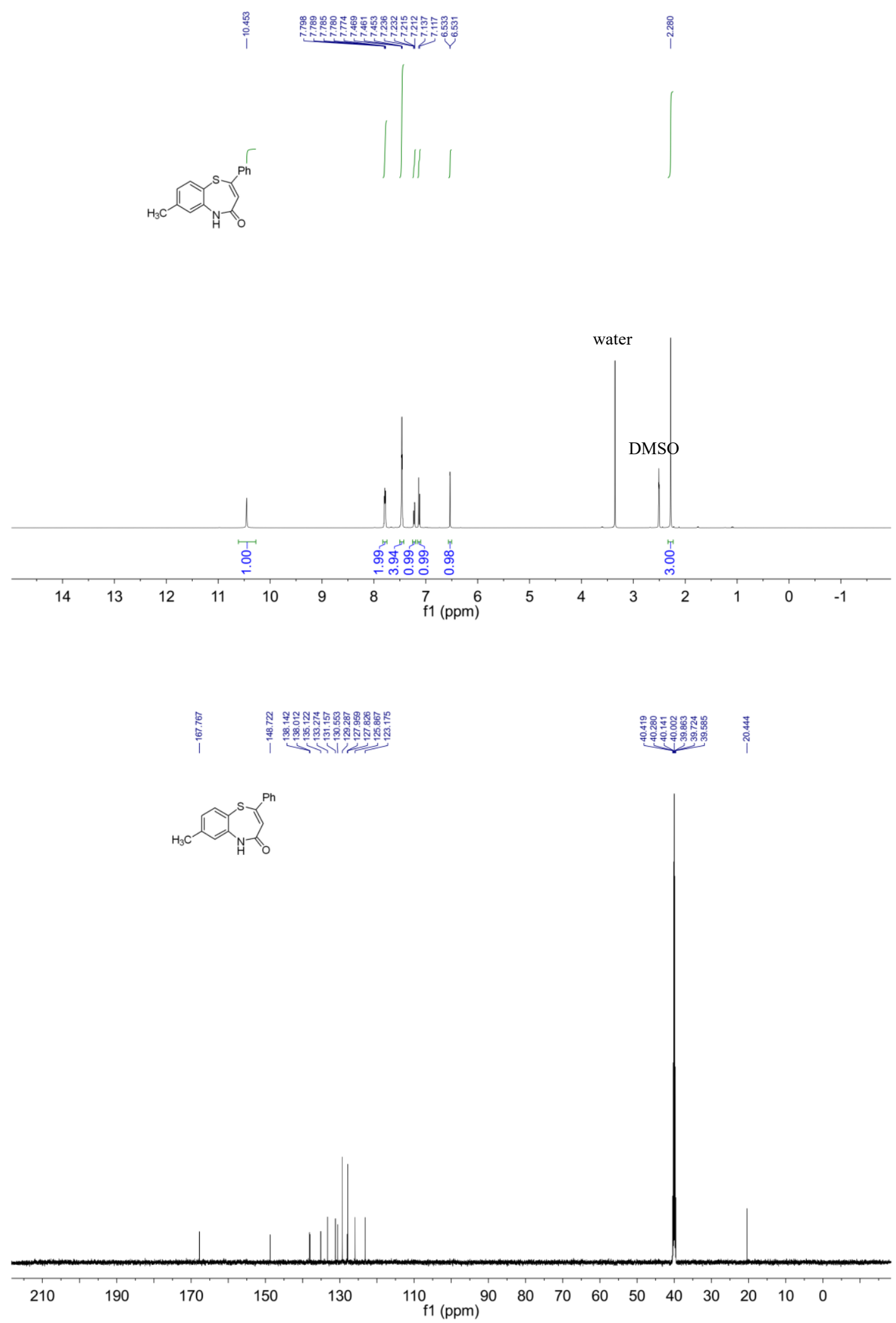
6-methyl-2-phenylbenzo[b][1,4]thiazepin-4(5H)-one $\mathbf{1 g}$
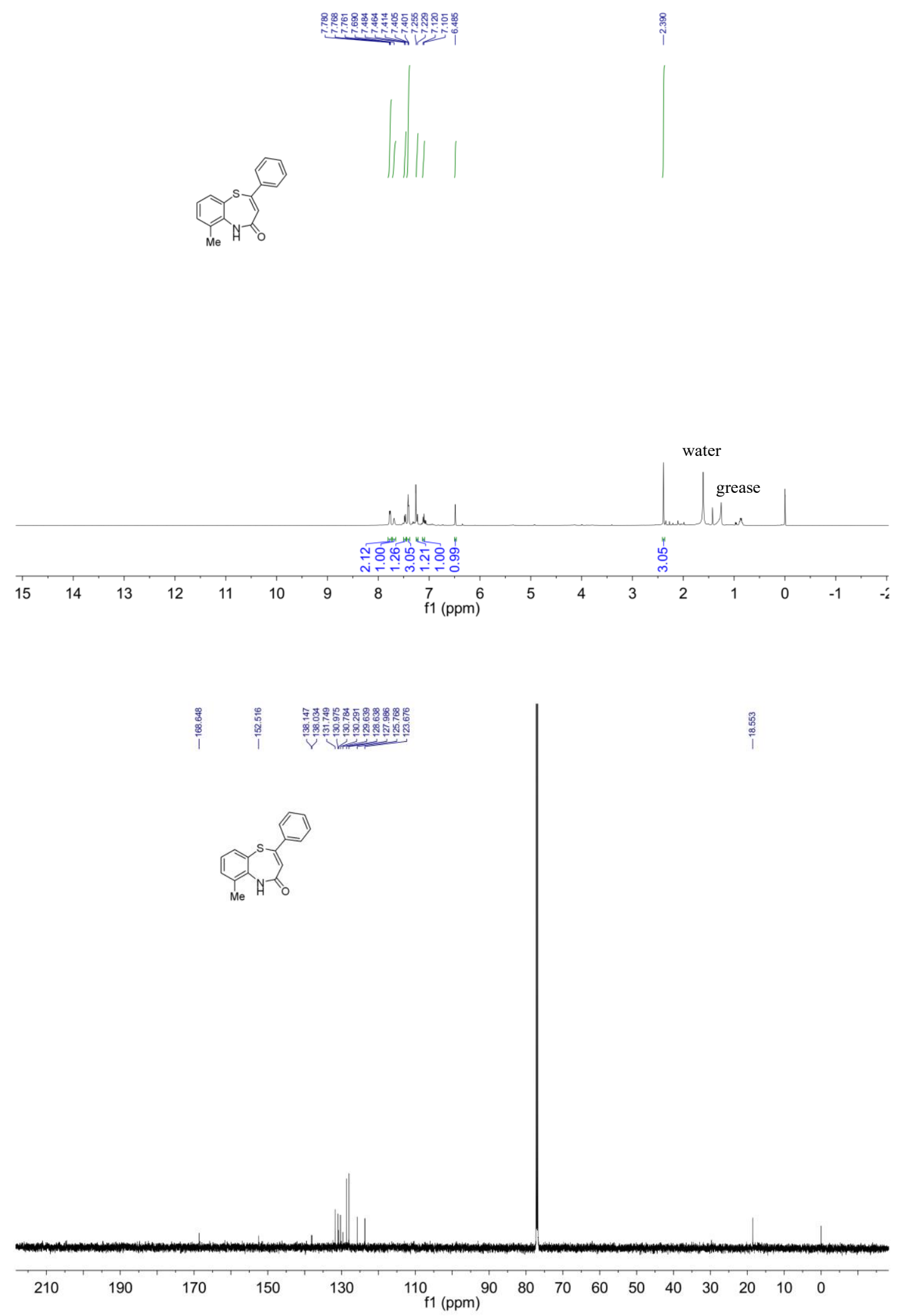
8-methoxy-2-phenylbenzo[b][1,4]thiazepin-4(5H)-one $\mathbf{1 h}$
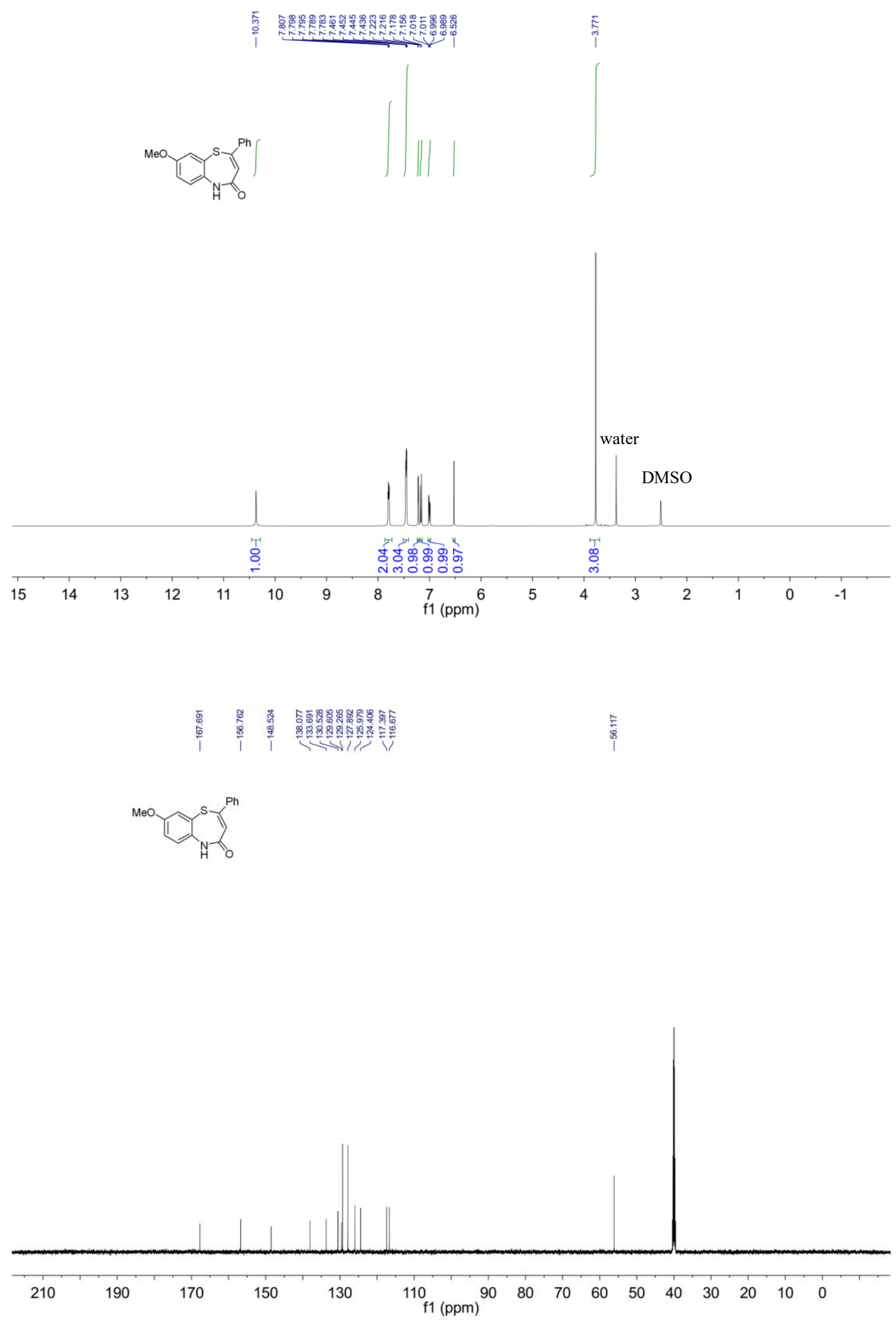
2-(4-fluorophenyl)benzo[b][1,4]thiazepin-4(5H)-one 1i
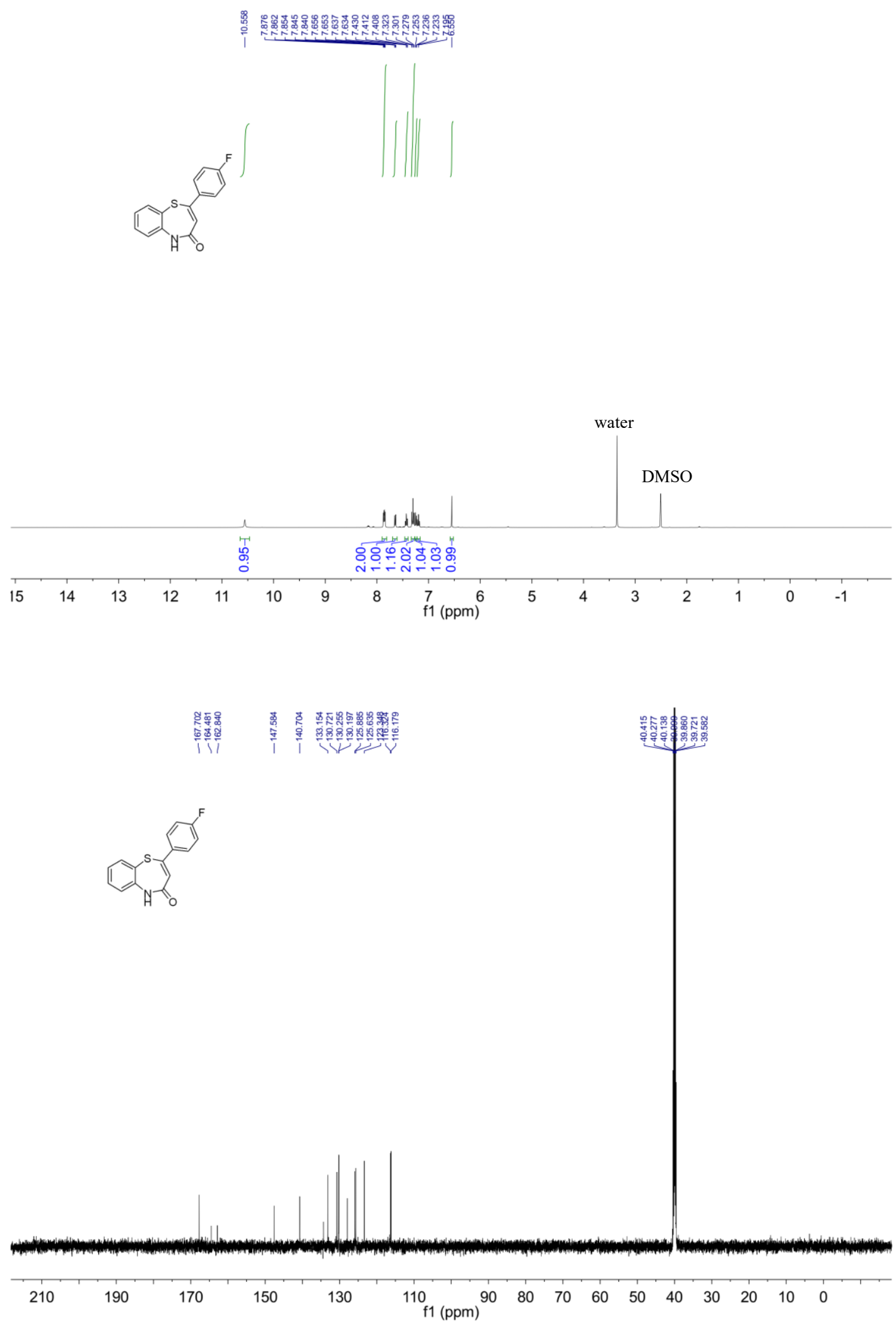
2-(4-chlorophenyl)benzo[b][1,4]thiazepin-4(5H)-one $\mathbf{1 j}$
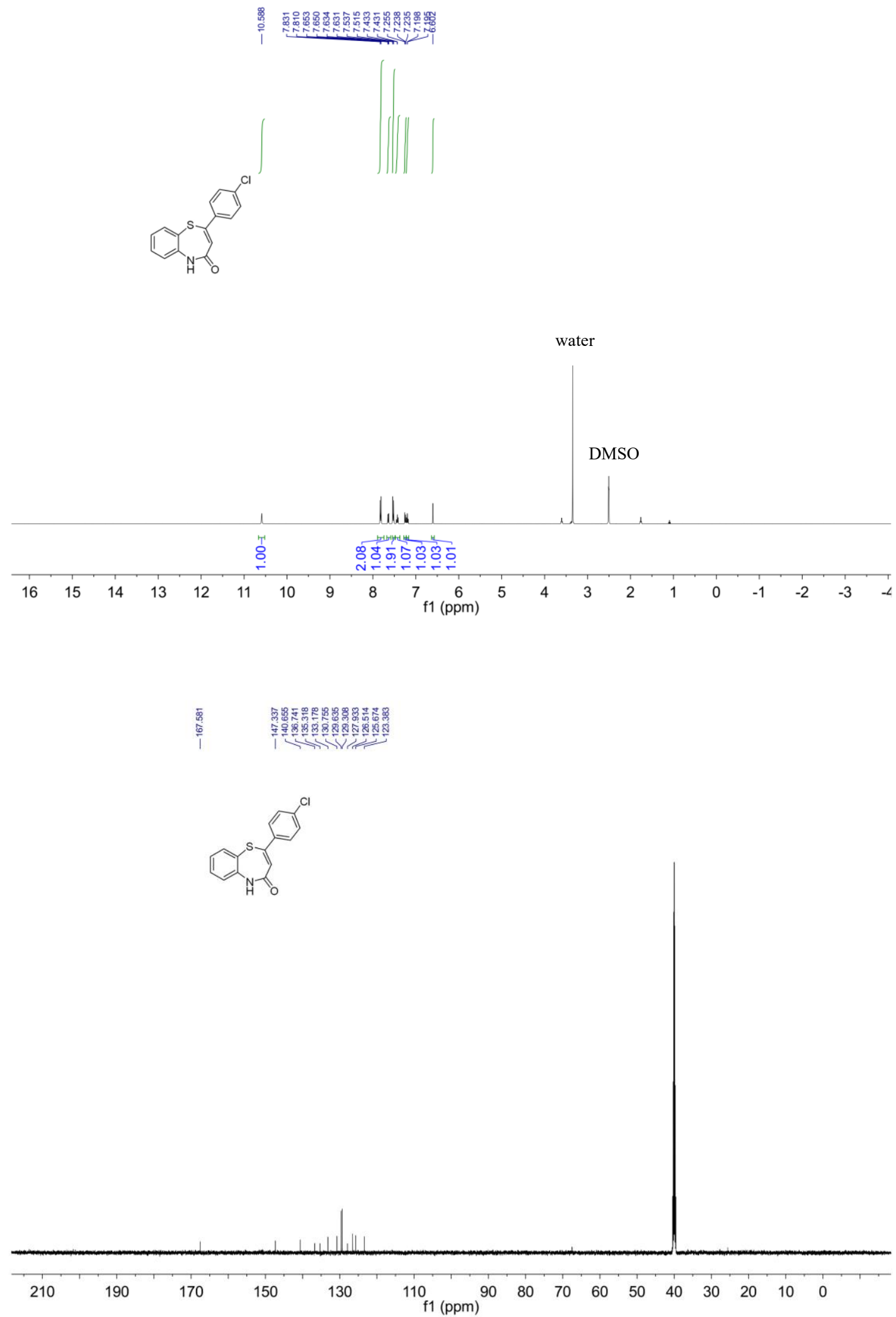
2-(4-(trifluoromethyl)phenyl)benzo[b][1,4]thiazepin-4(5H)-one $\mathbf{1 k}$

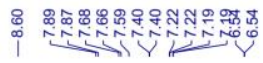
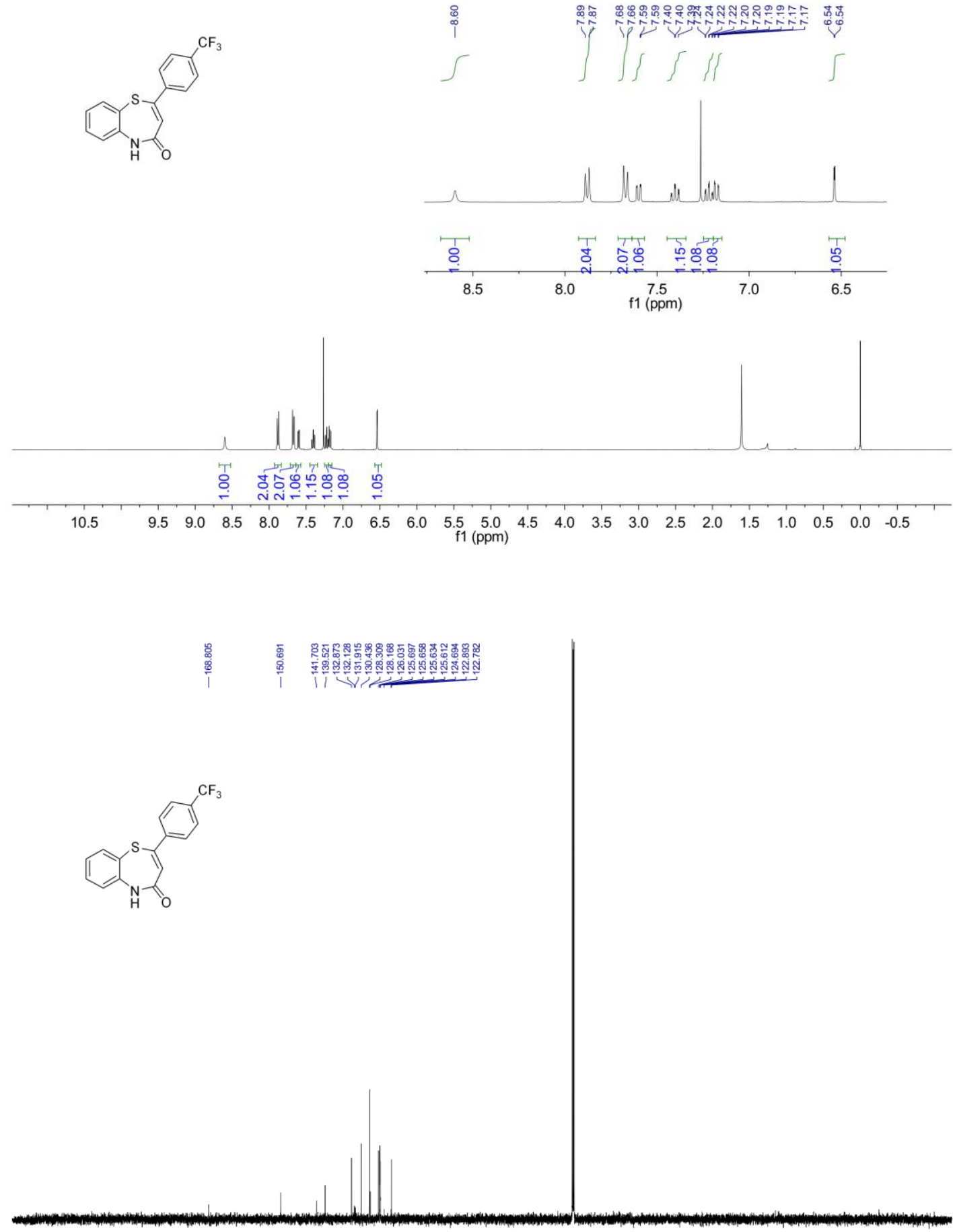

f1 $(\mathrm{ppm})$

210

190

170

150

130

110 f1 (ppm) 90

$\begin{array}{lllllllll}80 & 70 & 60 & 50 & 40 & 30 & 20 & 10 & 0\end{array}$ 
methyl 4-(4-oxo-4,5-dihydrobenzo[b][1,4]thiazepin-2-yl)benzoate $\mathbf{1 l}$
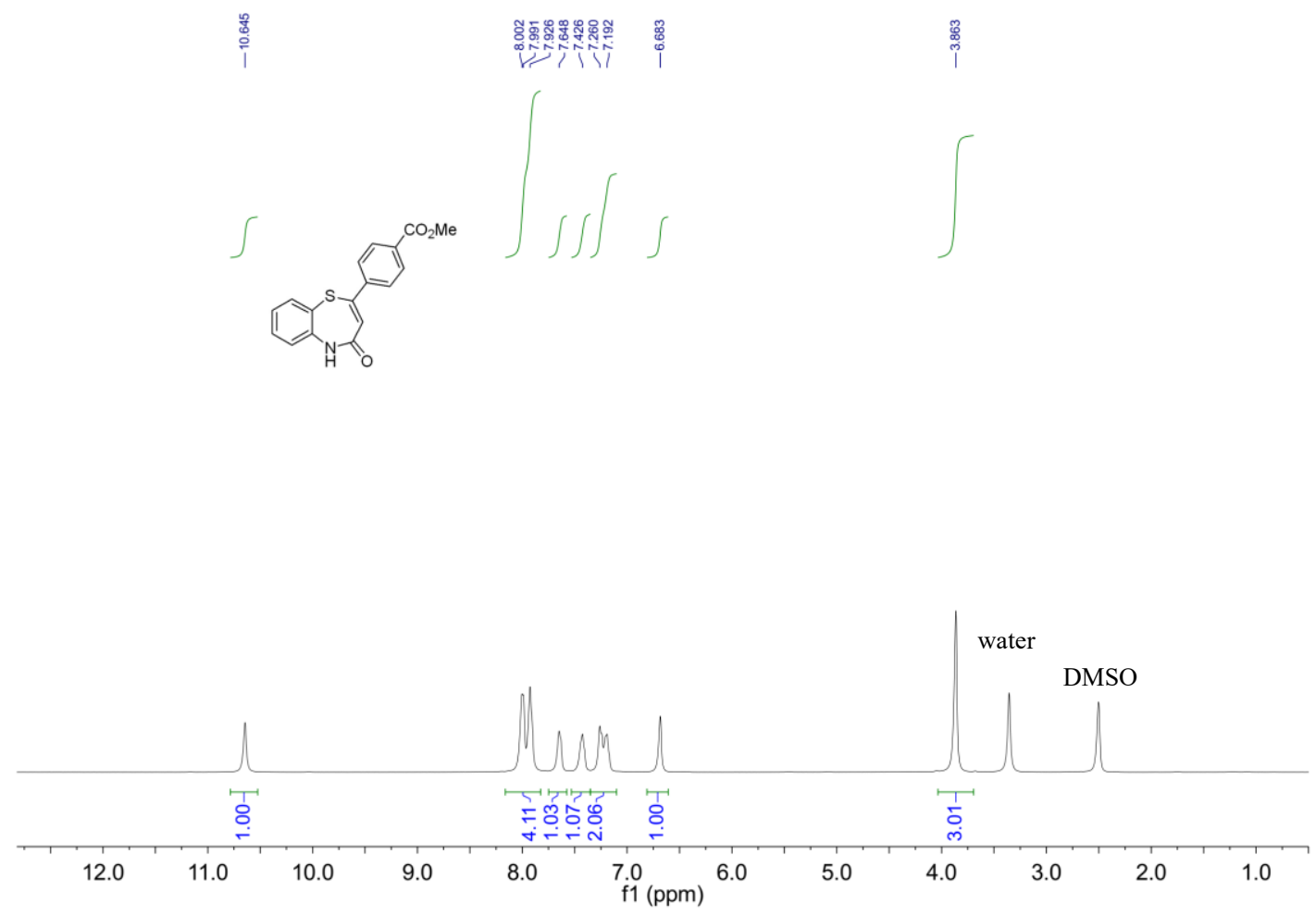

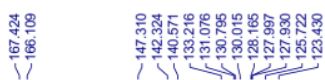
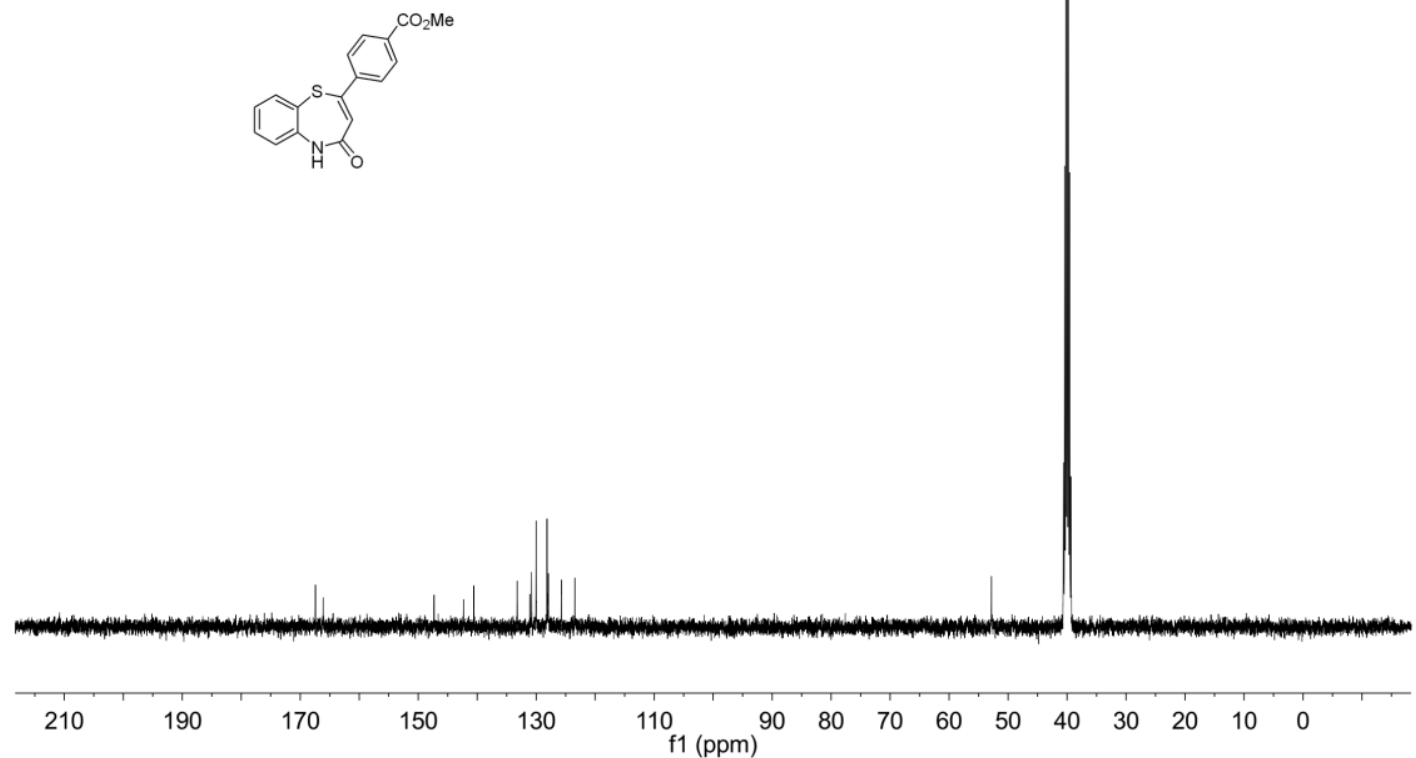
2-(4-methoxyphenyl)benzo[b][1,4]thiazepin-4(5H)-one 1m

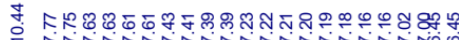

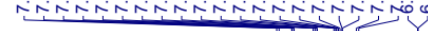<smiles>O=C1C(=C2Nc3ccccc3S2)C=CC1O</smiles>
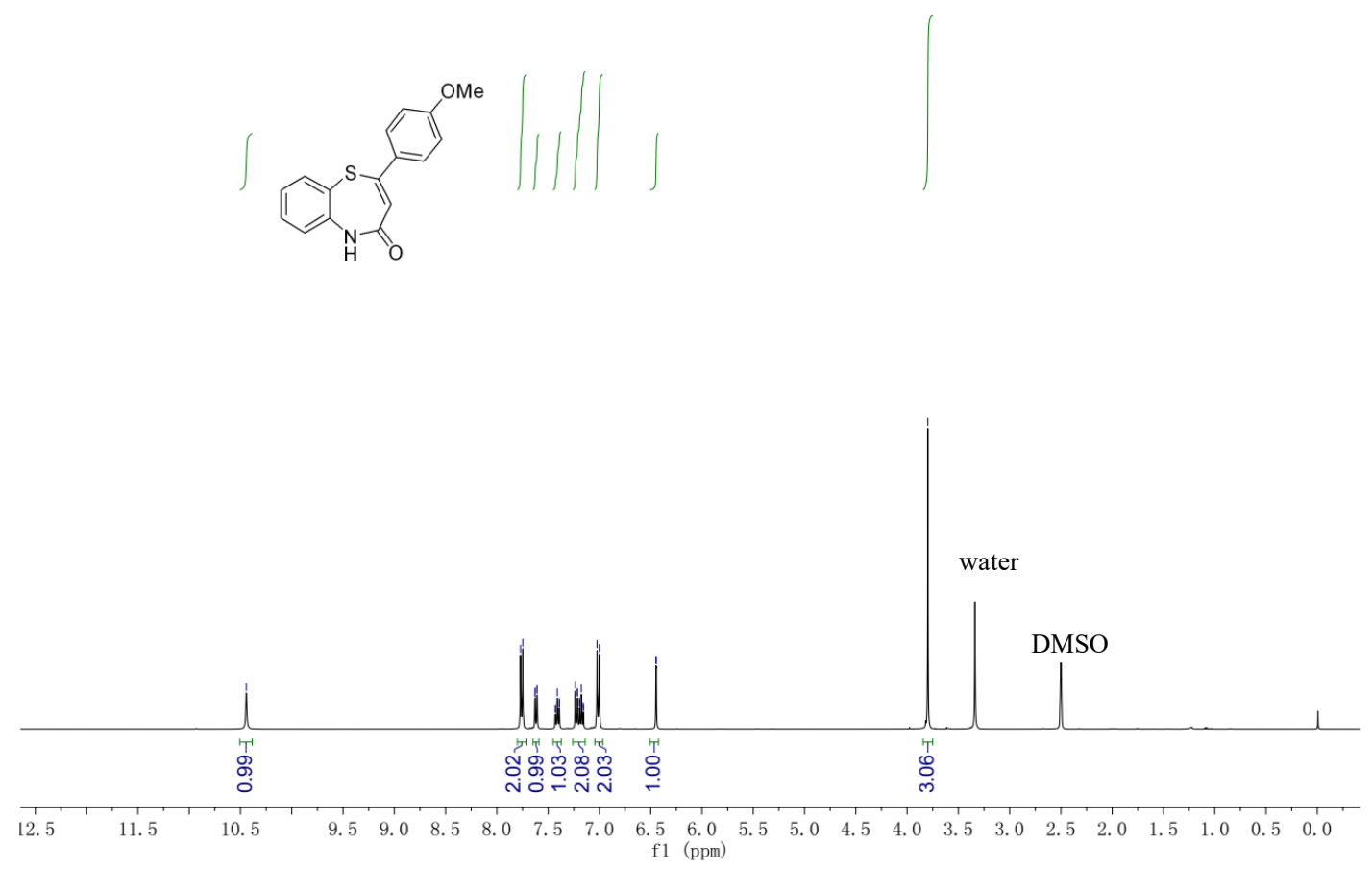

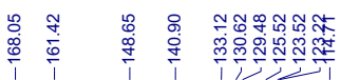

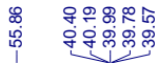<smiles>COc1ccc(-c2cc(=O)[nH]c3ccccc23)cc1</smiles>

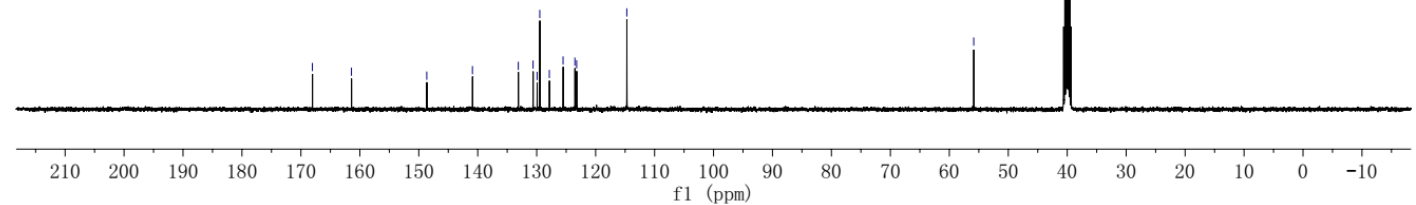


7-chloro-2-(4-methoxyphenyl)benzo[b][1,4]thiazepin-4(5H)-one 1n
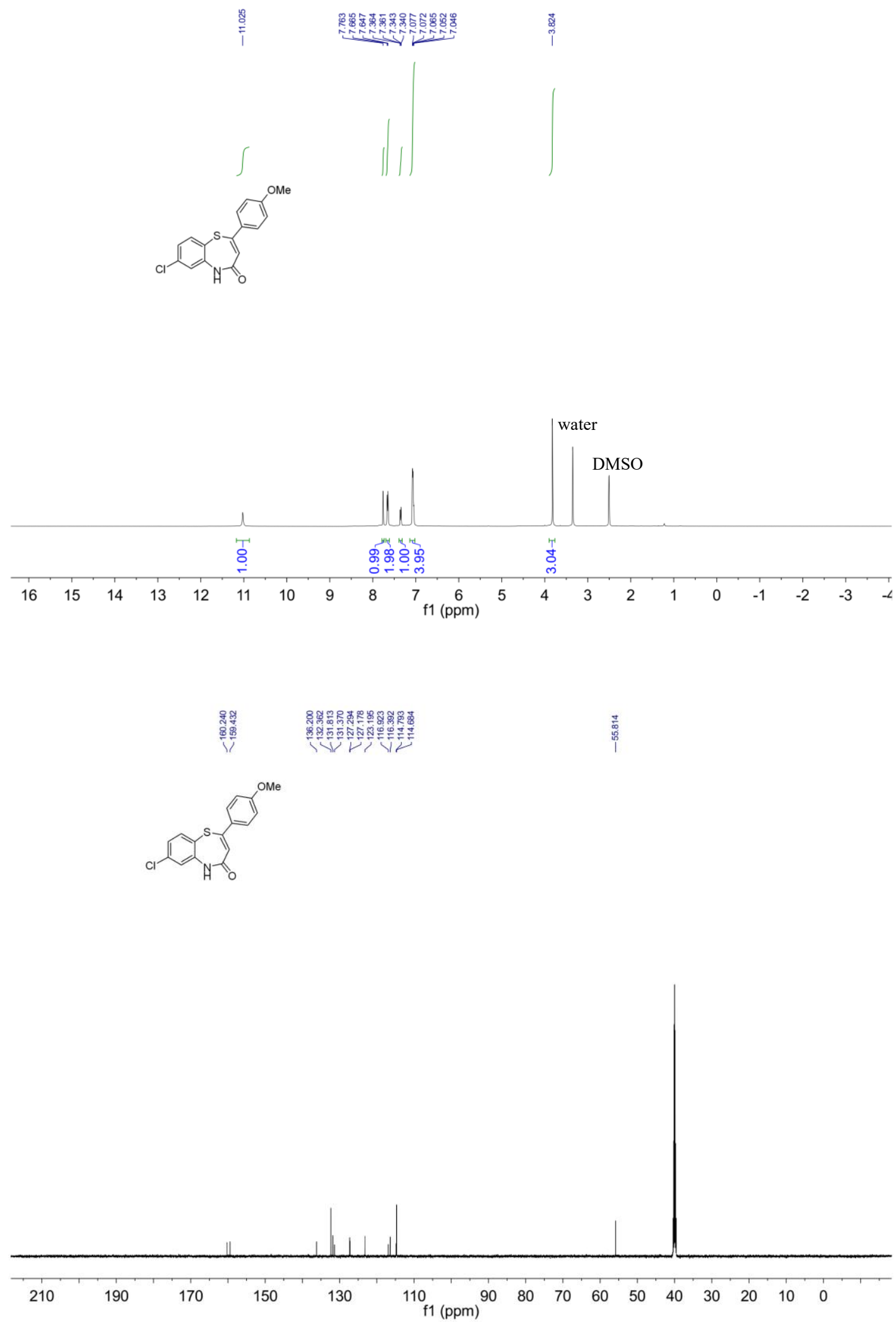
2-methylbenzo $[b][1,4]$ thiazepin-4(5H)-one 1o
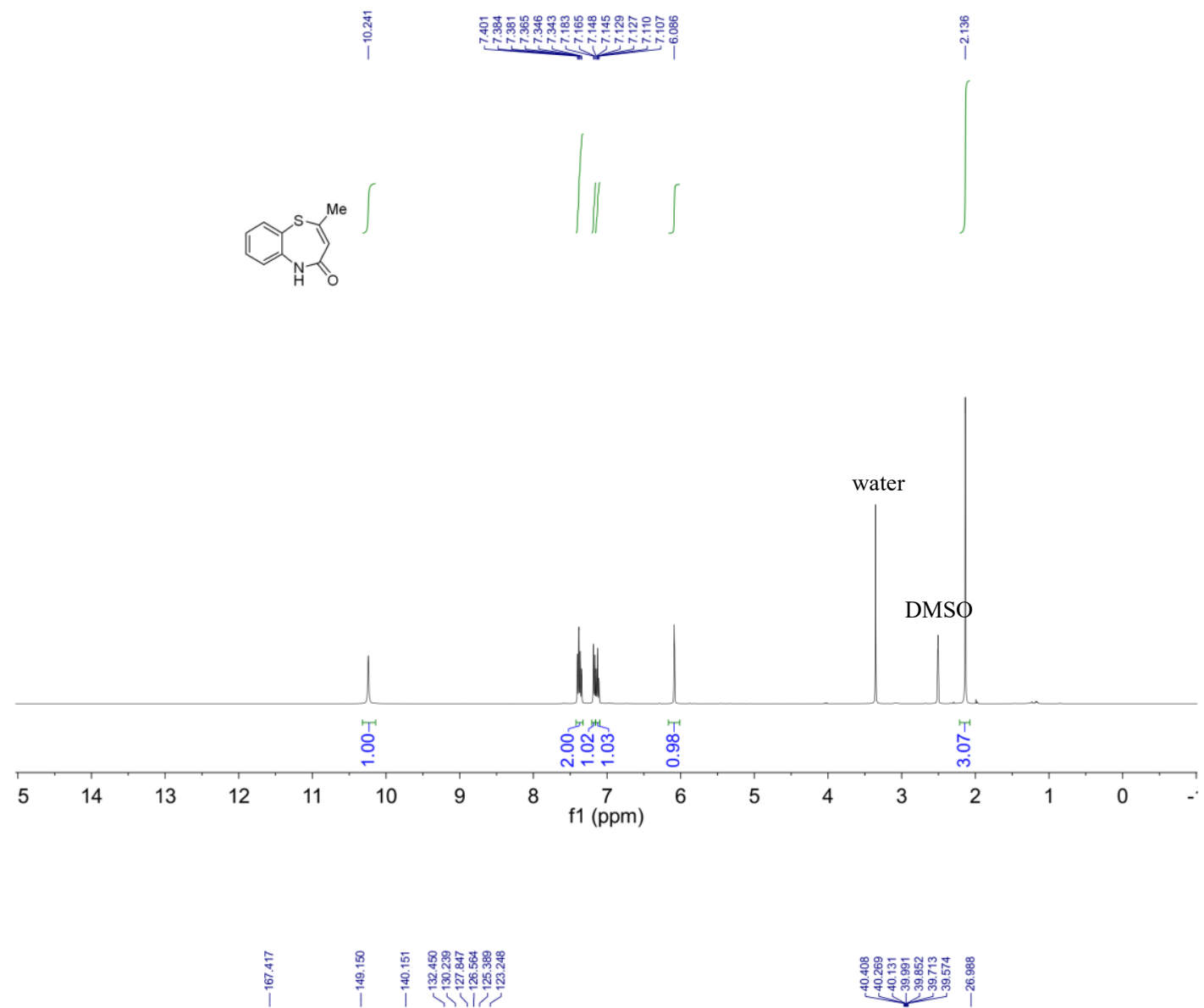

$\overbrace{H}^{S}$

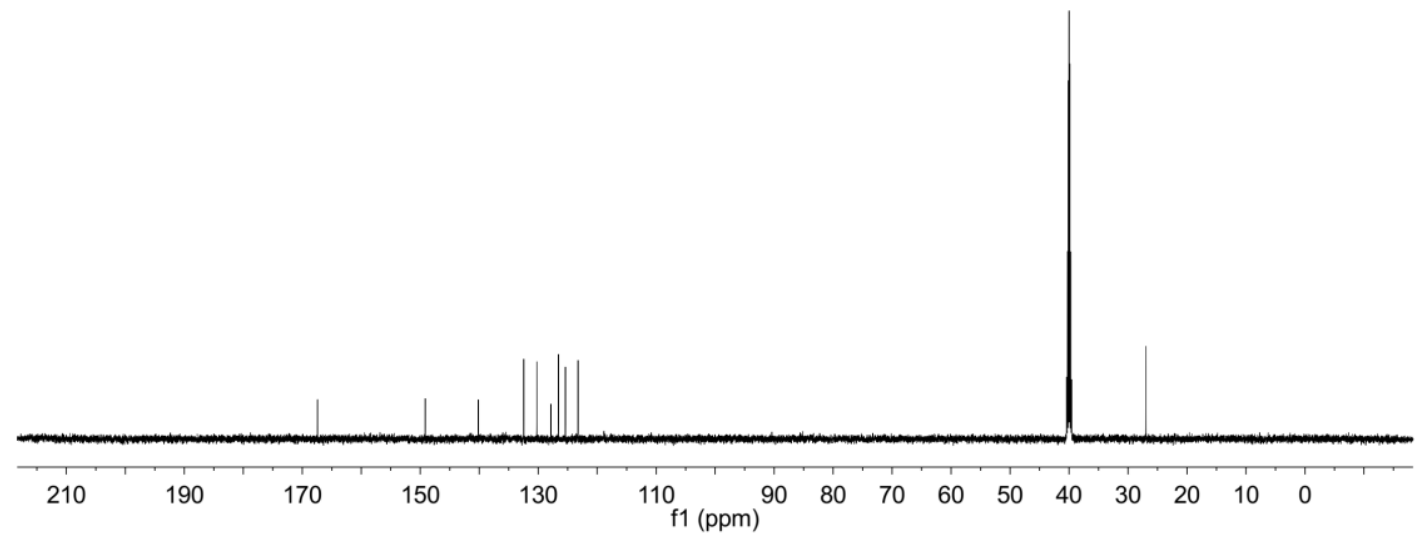


(R)-2-phenyl-2,3-dihydrobenzo[b][1,4]thiazepin-4(5H)-one 2a
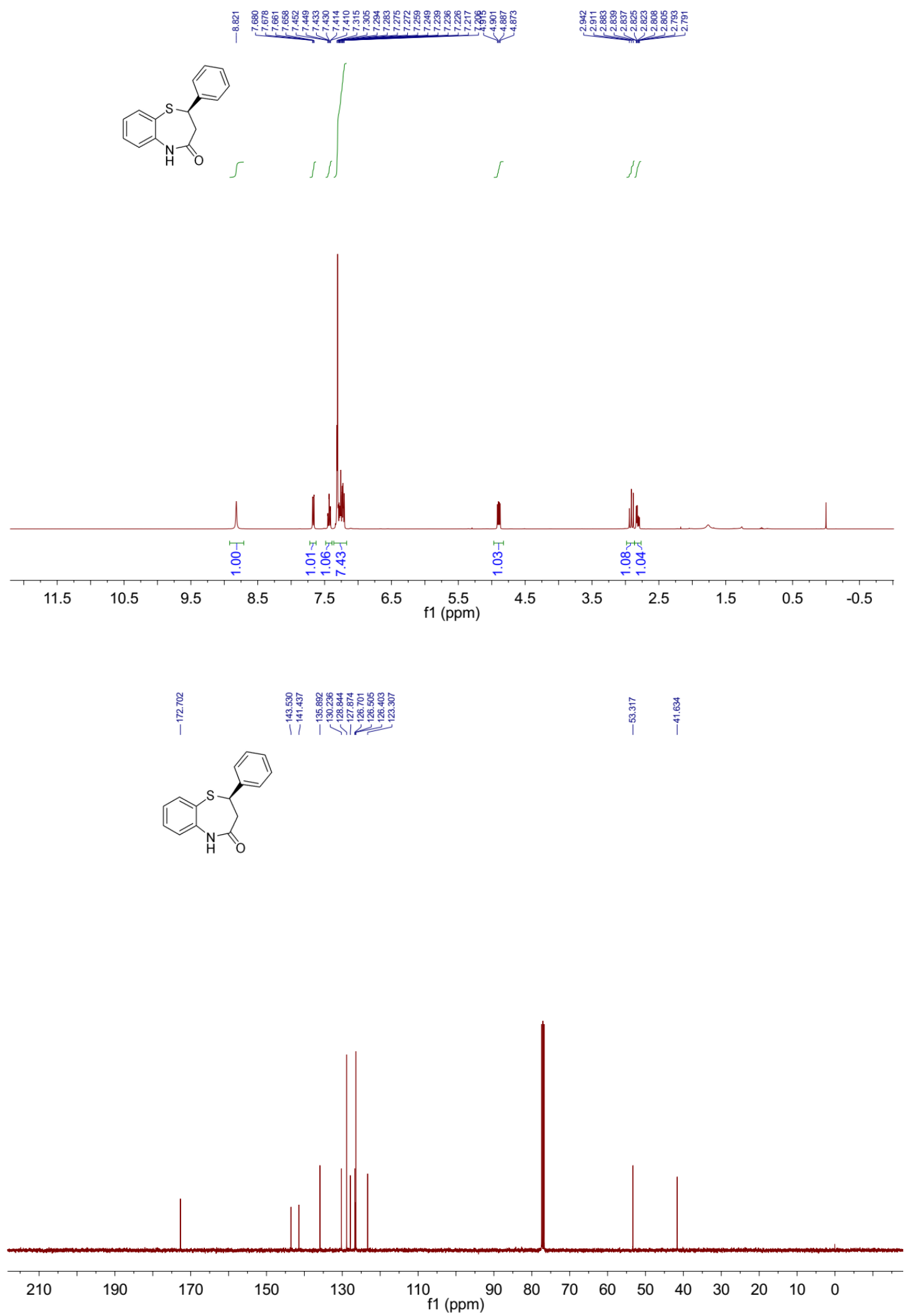
(R)-5-methyl-2-phenyl-2,3-dihydrobenzo[b][1,4]thiazepin-4(5H)-one $\mathbf{2 b}$

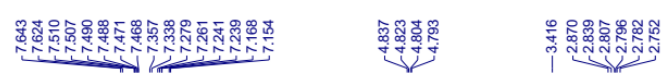<smiles>CN1C(=O)CC(c2ccccc2)Sc2ccccc21</smiles>
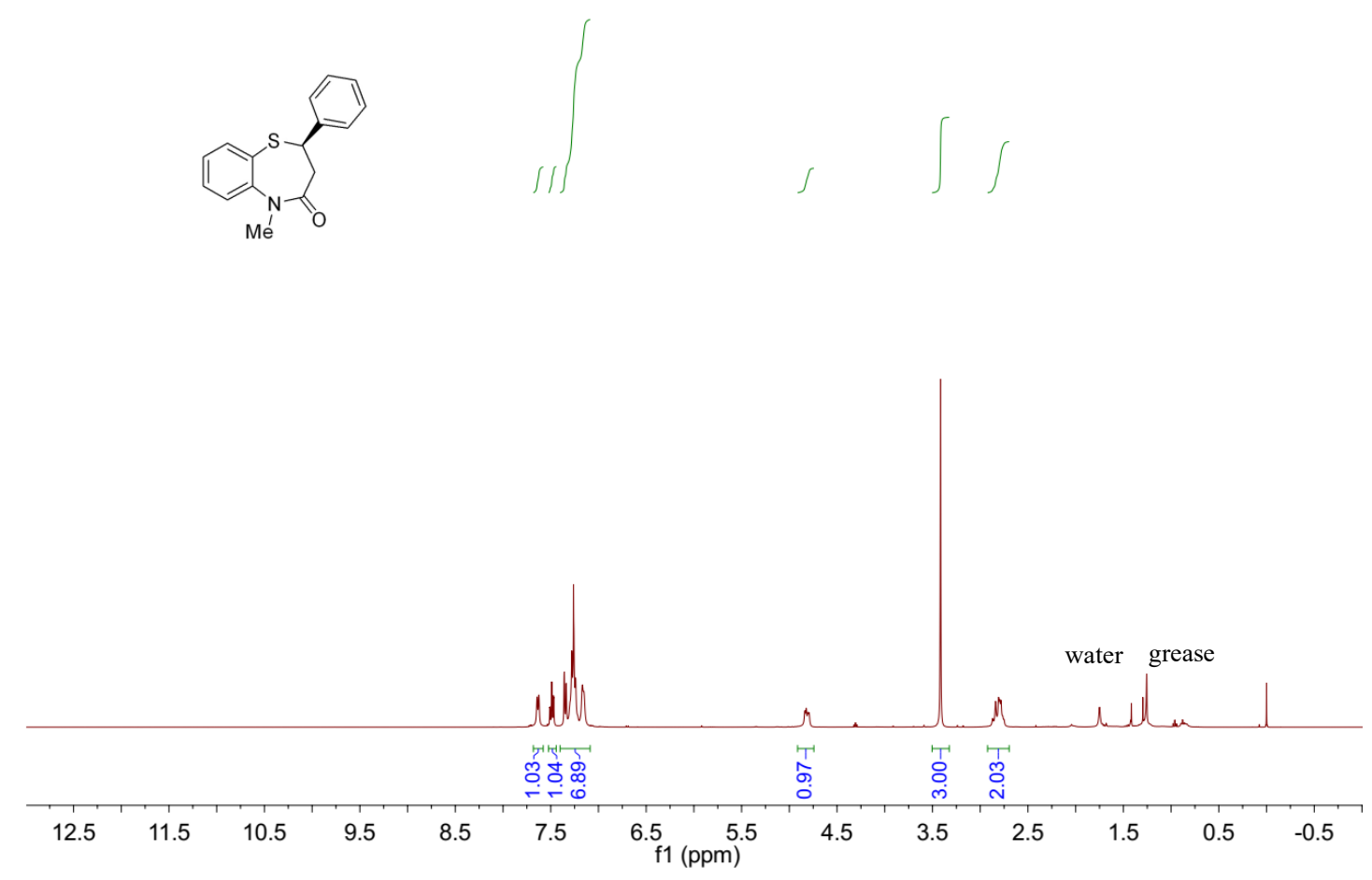

$\begin{array}{lll}1 & 1 \\ 0 & 1\end{array}$

11<smiles>CN1C(=O)CC(c2ccccc2)Sc2ccccc21</smiles>

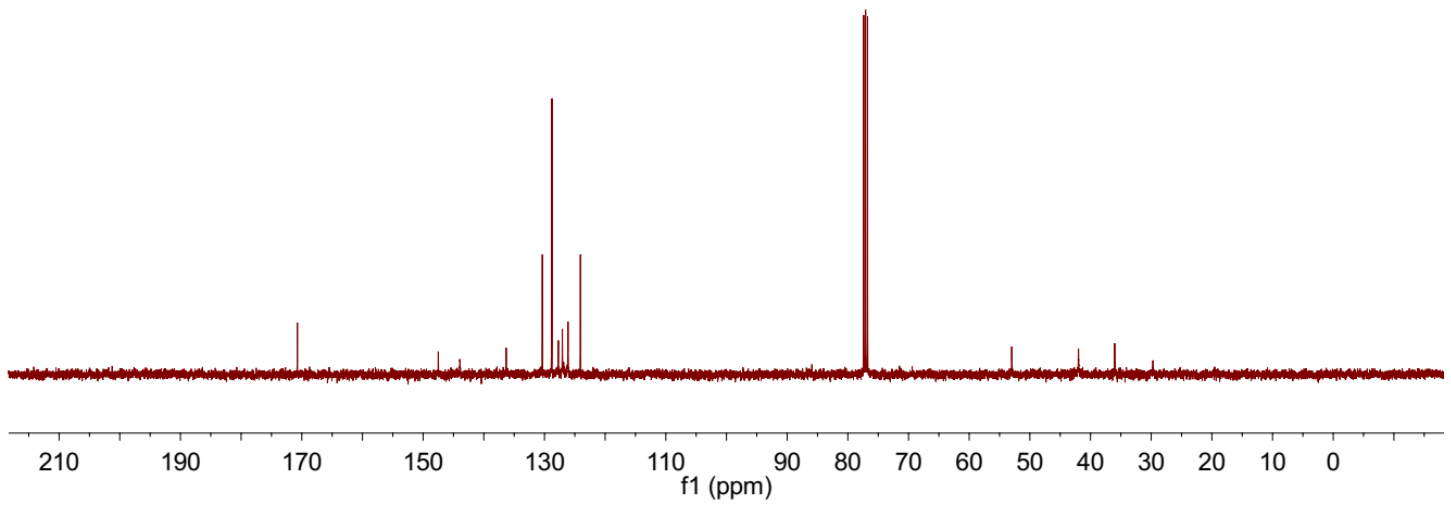


(R)-5-benzyl-2-phenyl-2,3-dihydrobenzo[b][1,4]thiazepin-4(5H)-one 2c

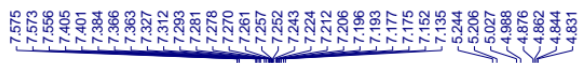

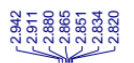<smiles>O=C1CC(c2ccccc2)Sc2ccccc21</smiles>
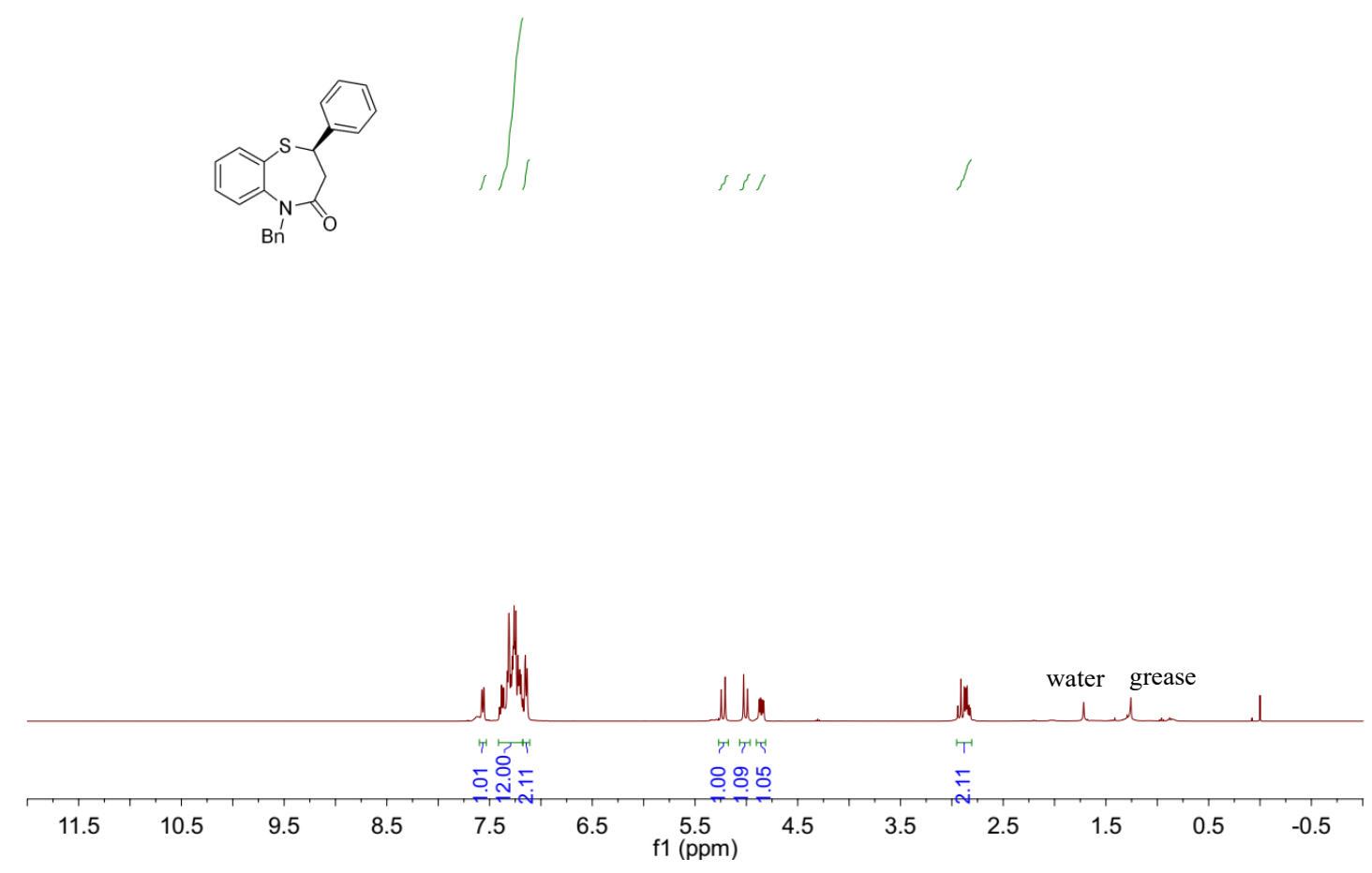

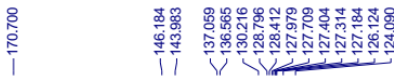

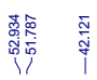<smiles>O=C1CC(c2ccccc2)Sc2ccccc21</smiles>

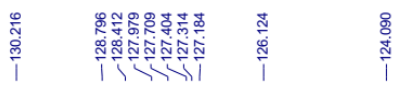
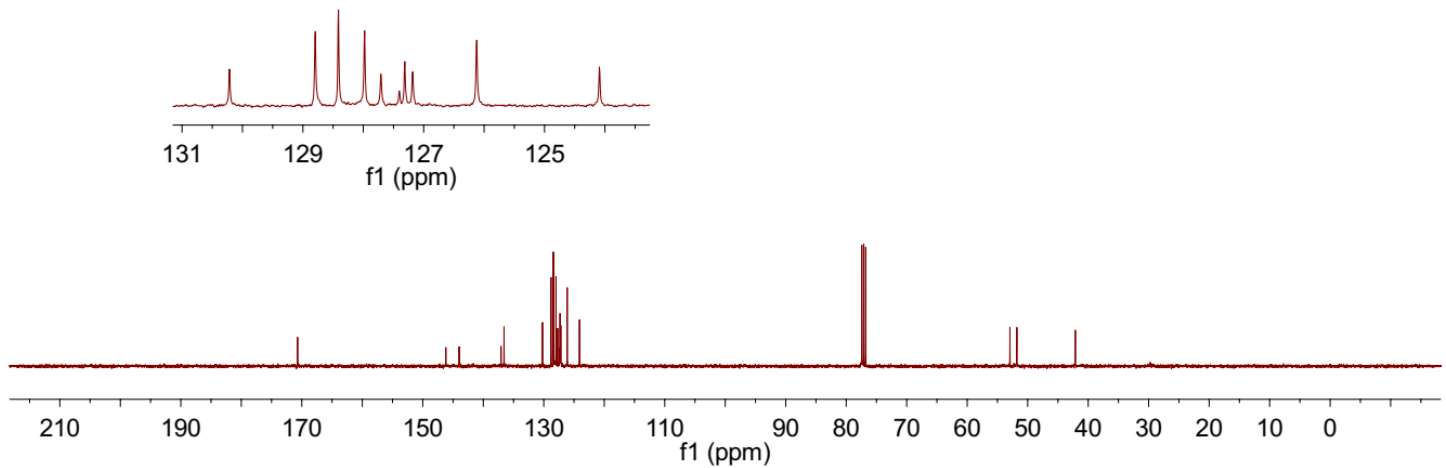
(R)-7-fluoro-2-phenyl-2,3-dihydrobenzo[b][1,4]thiazepin-4(5H)-one 2d

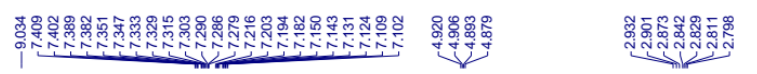

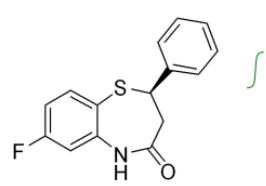

|| ||
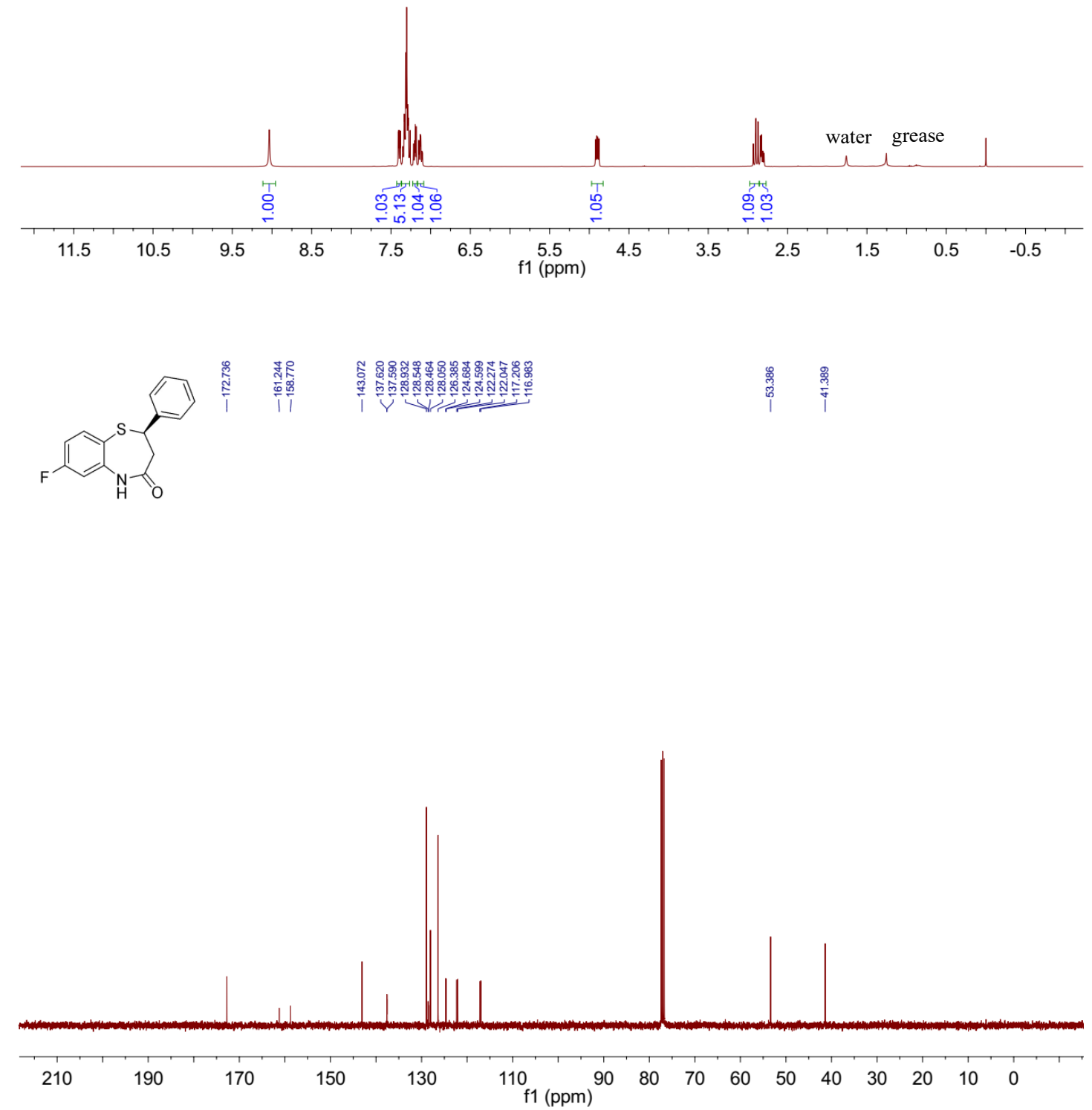
(R)-7-chloro-2-phenyl-2,3-dihydrobenzo[b][1,4]thiazepin-4(5H)-one $2 \mathbf{e}$

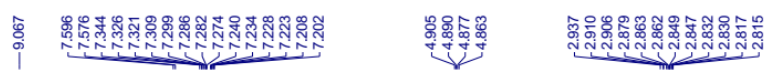<smiles>O=C1CC(c2ccccc2)Sc2ccc(Cl)cc2N1</smiles><smiles>c1ccccc#1</smiles>
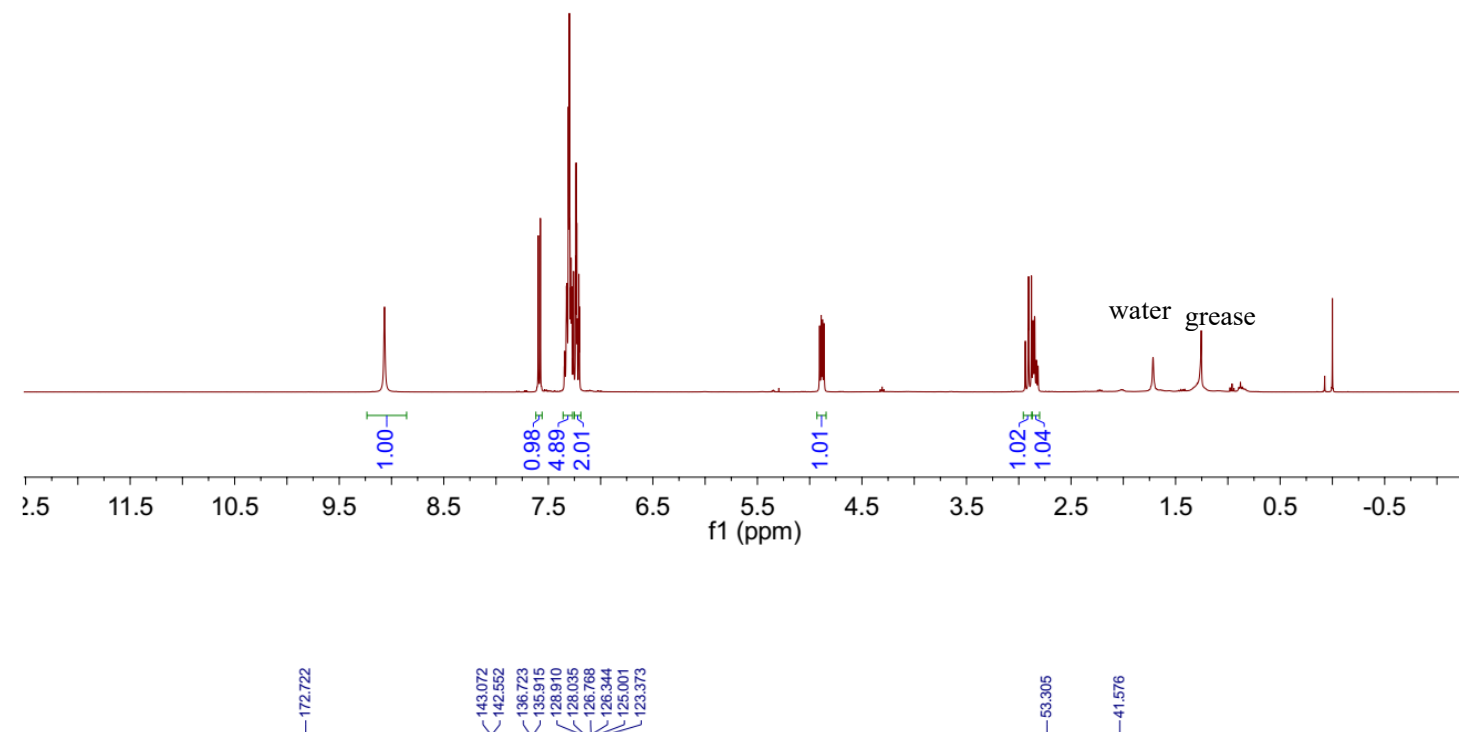<smiles>O=C1Nc2cc(Cl)ccc2SC1c1ccccc1</smiles>

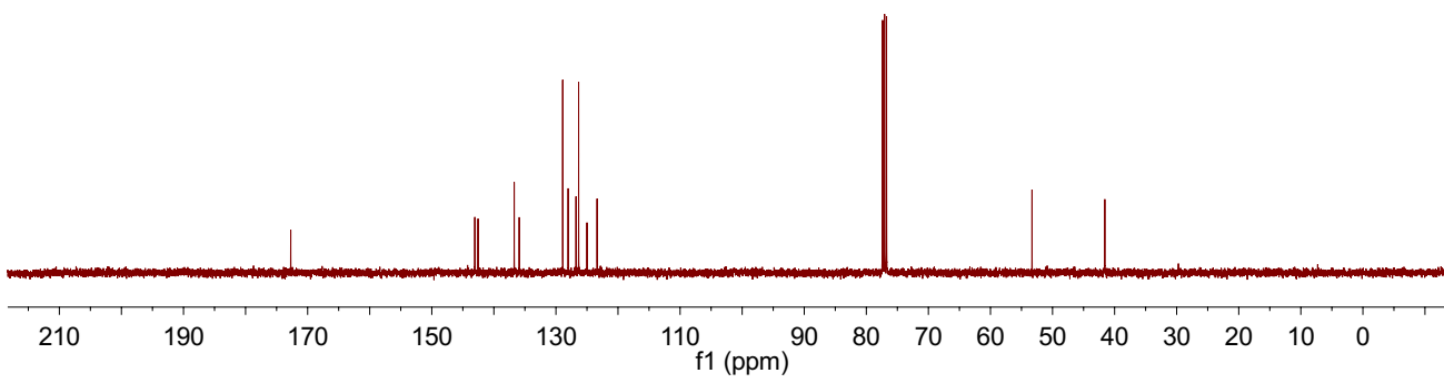


(R)-7-methyl-2-phenyl-2,3-dihydrobenzo[ $b][1,4]$ thiazepin-4(5H)-one $\mathbf{2 f}$

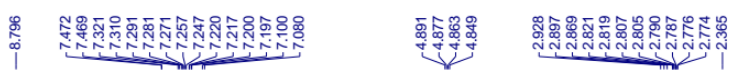
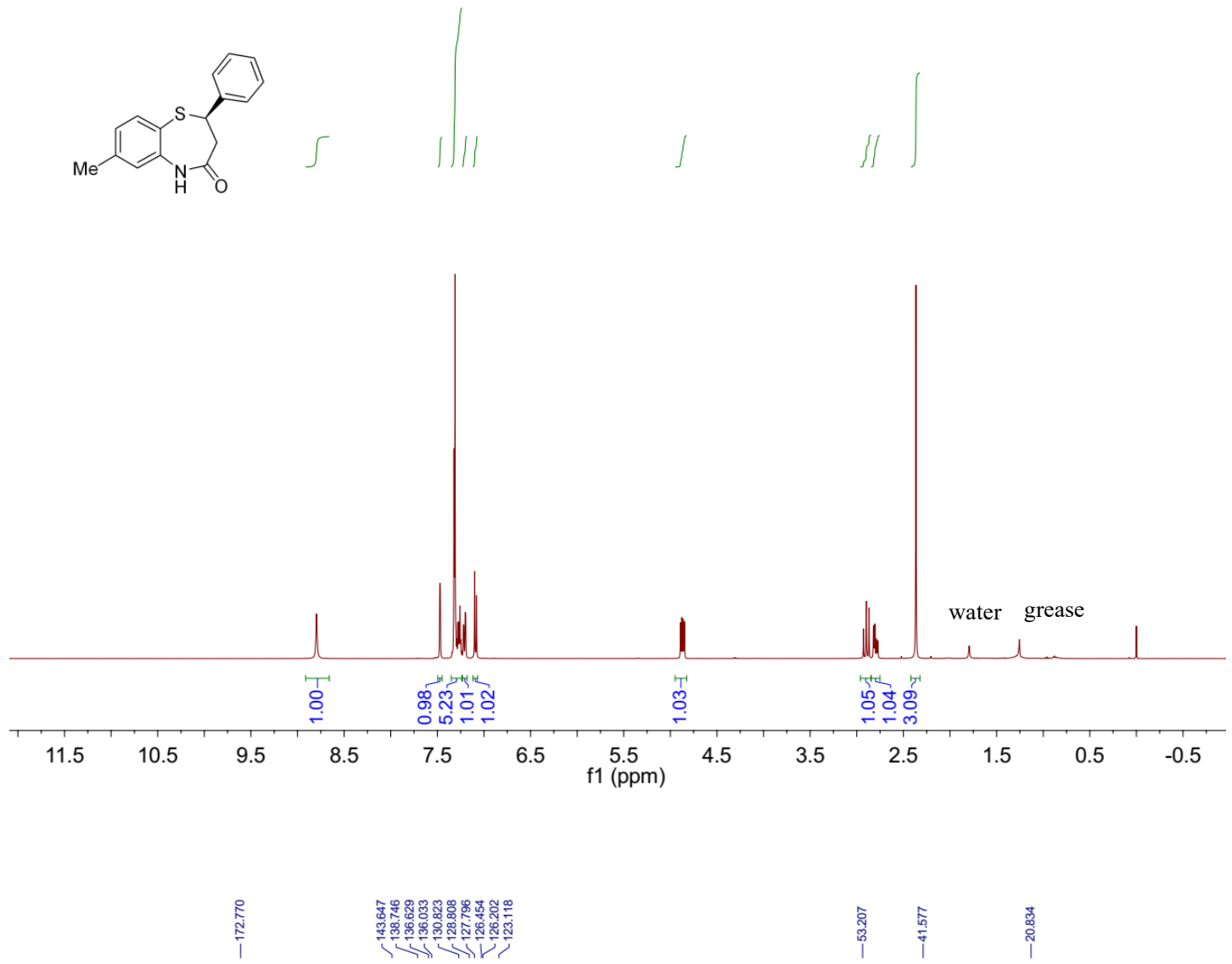

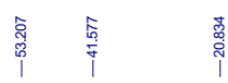
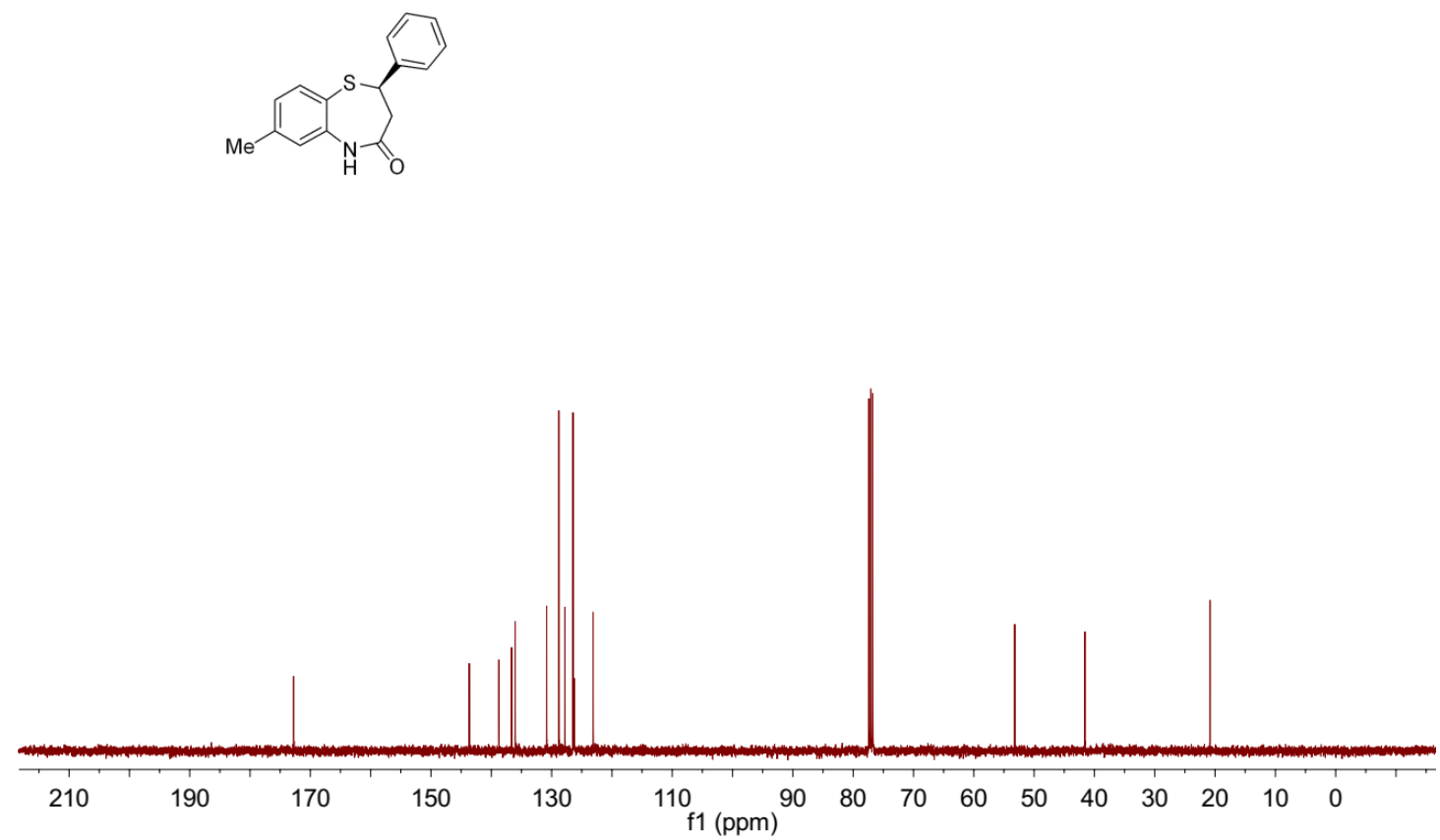
(R)-6-methyl-2-phenyl-2,3-dihydrobenzo[ $b][1,4]$ thiazepin-4(5H)-one $\mathbf{2 g}$

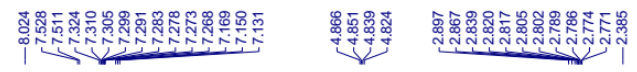<smiles>Nc1ccc2c(c1)NC(=O)CC2c1ccccc1</smiles>
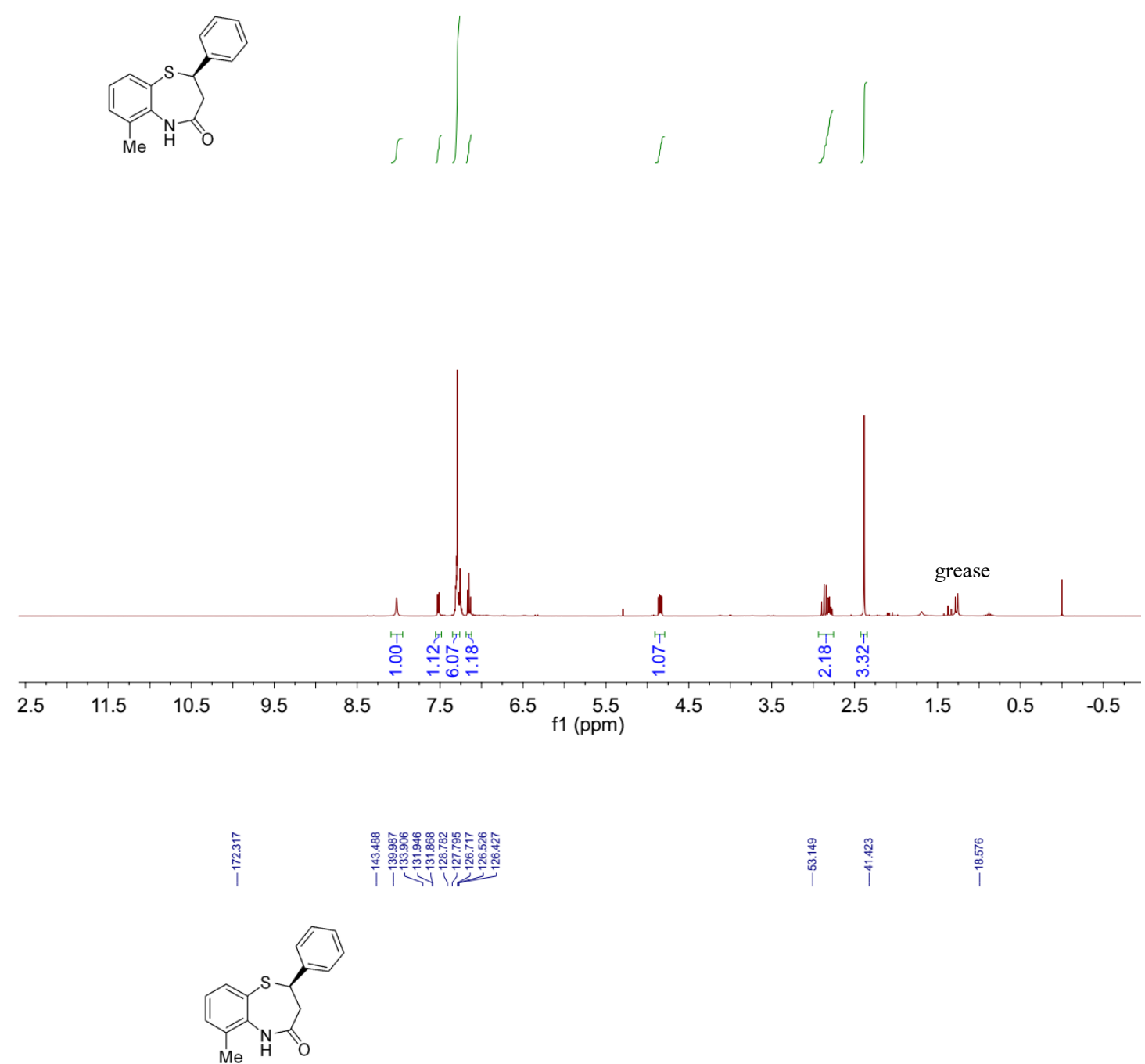

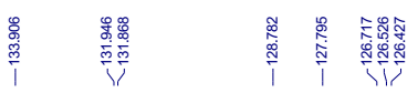
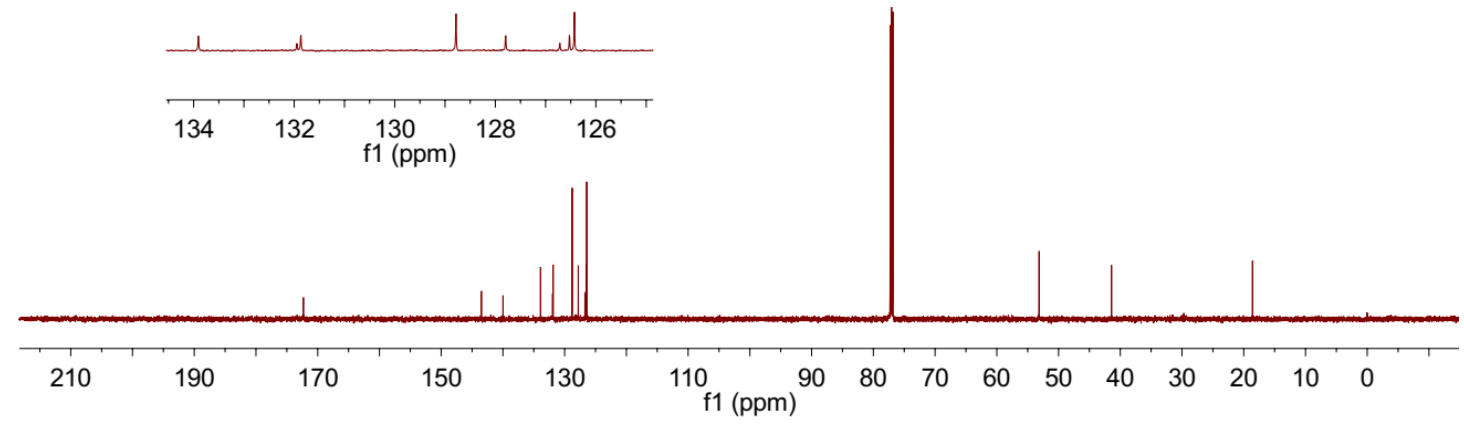
(R)-8-methoxy-2-phenyl-2,3-dihydrobenzo[b][1,4]thiazepin-4(5H)-one $\mathbf{2 h}$

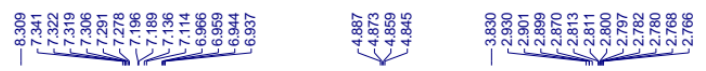<smiles>COc1ccc2c(c1)SC(c1ccccc1)CC(=O)N2</smiles>
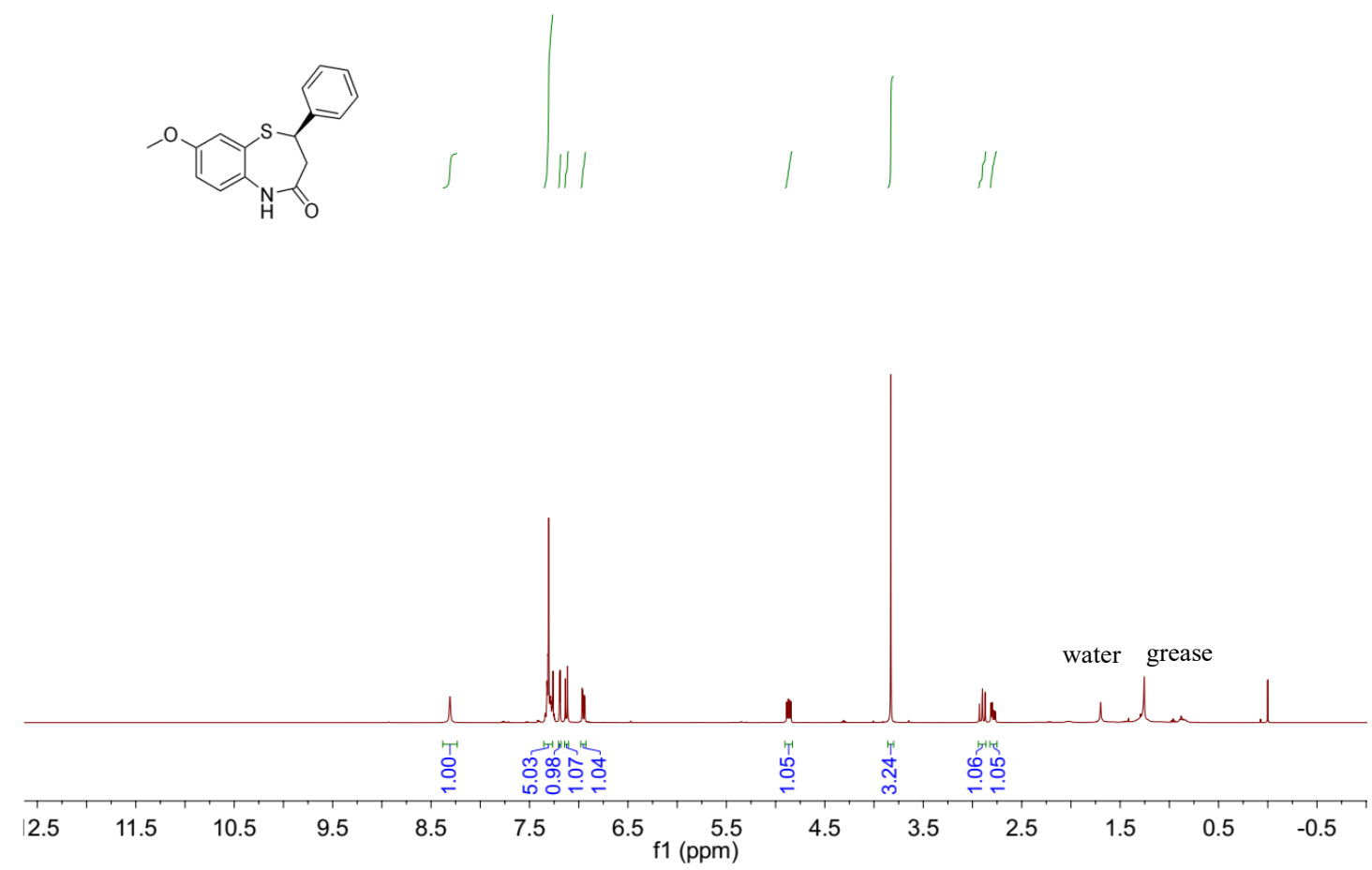

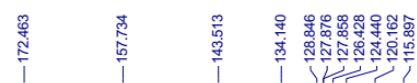
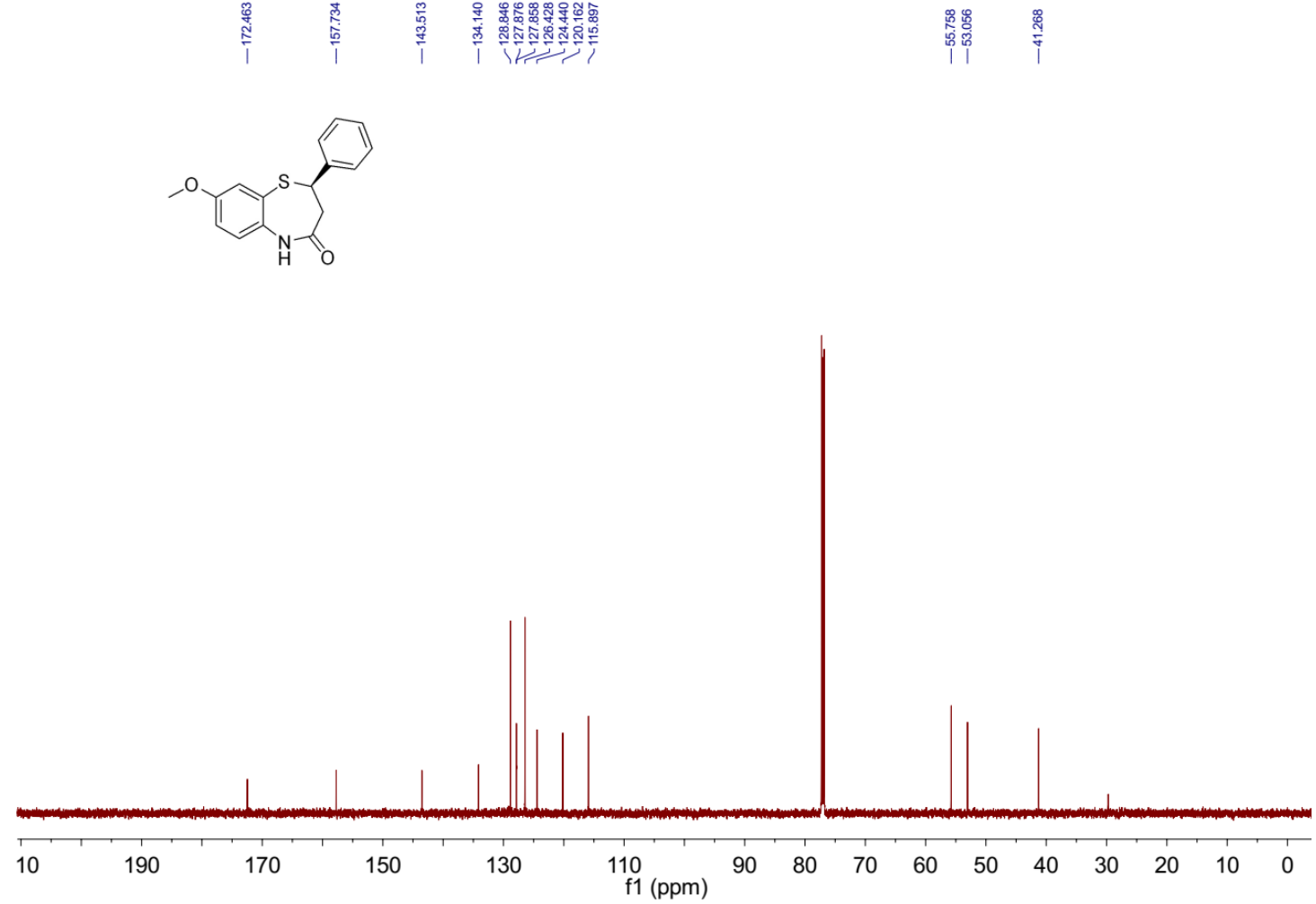
(R)-2-(4-fluorophenyl)-2,3-dihydrobenzo[b][1,4]thiazepin-4(5H)-one $\mathbf{2 i}$
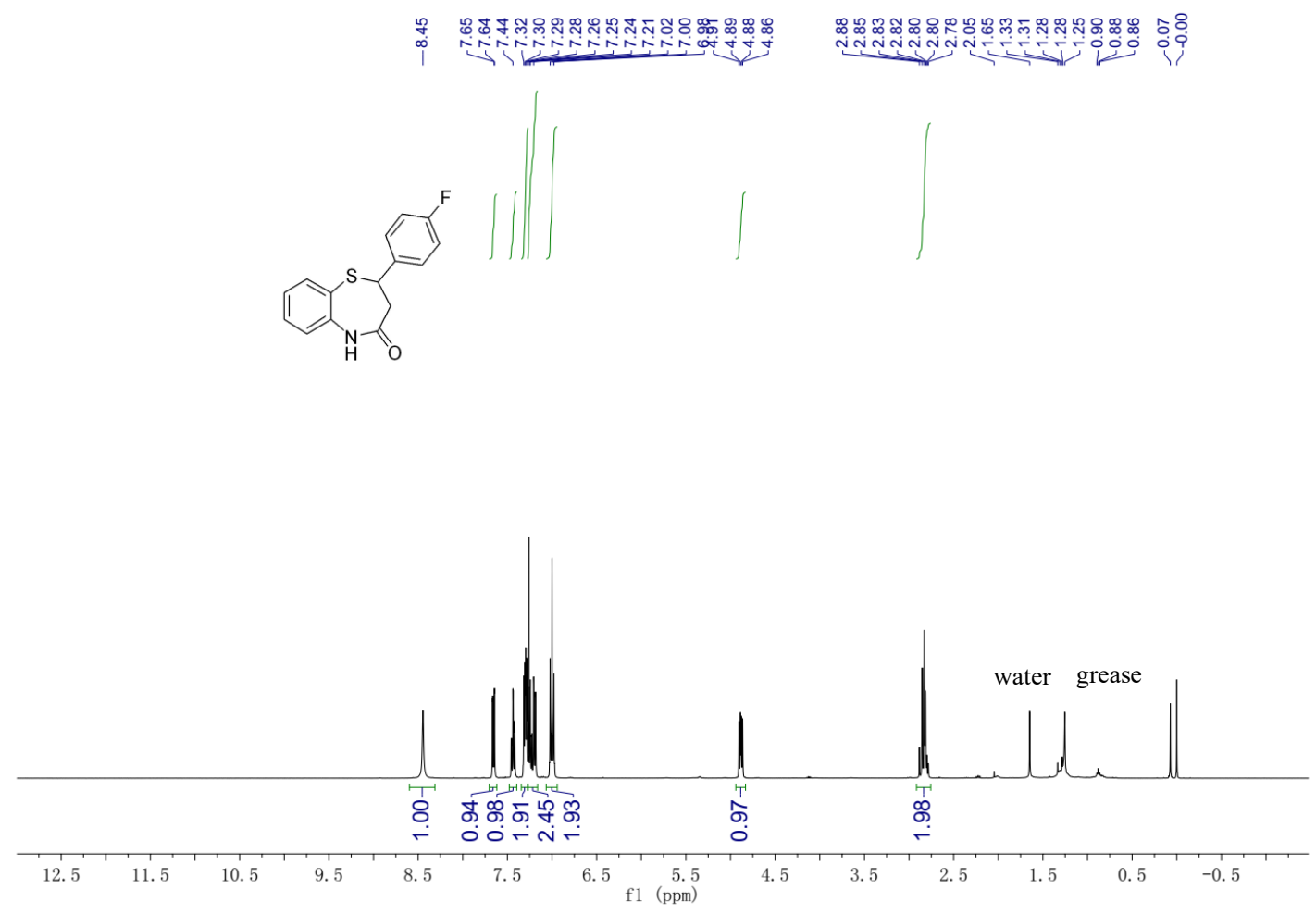

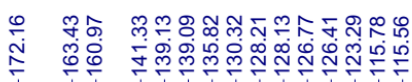

$\underset{\text { Îj }}{\text { i }}$
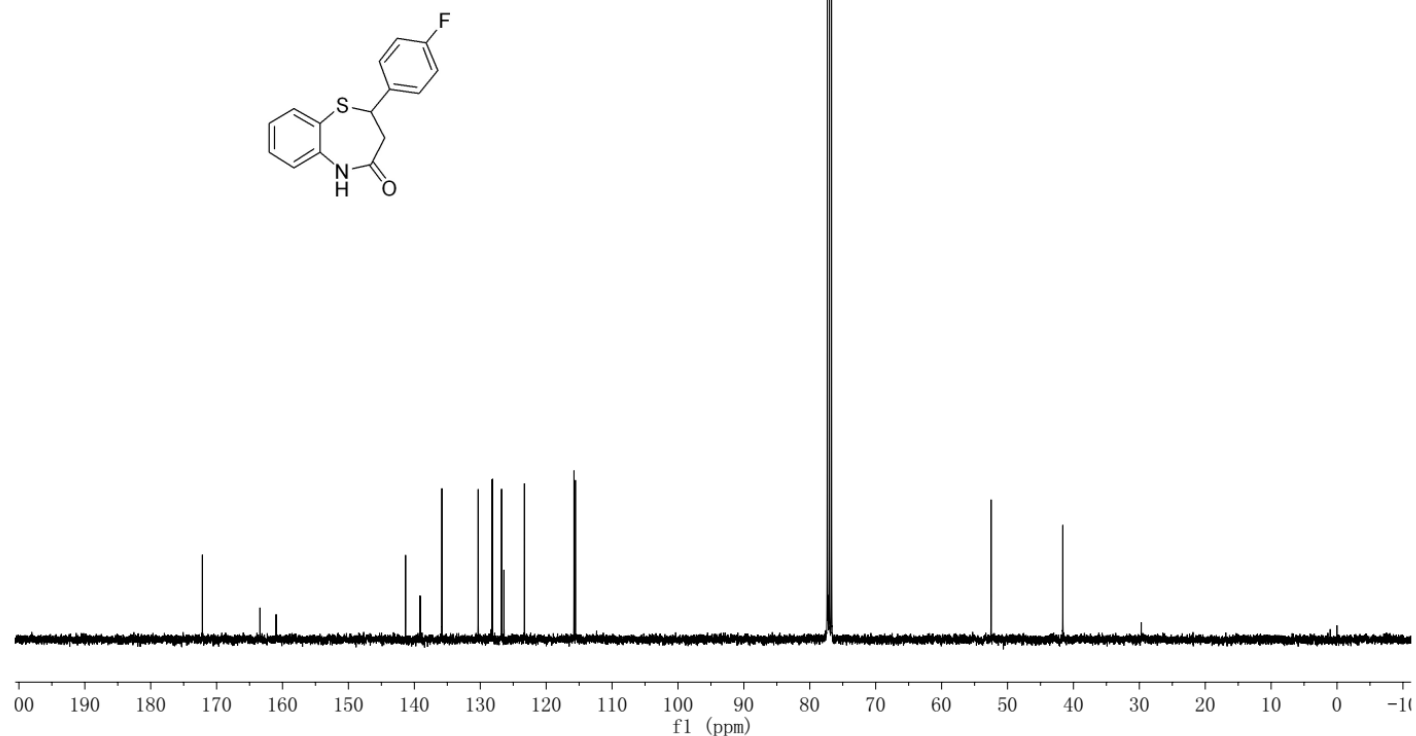
(R)-2-(4-chlorophenyl)-2,3-dihydrobenzo[b][1,4]thiazepin-4(5H)-one $\mathbf{2 j}$
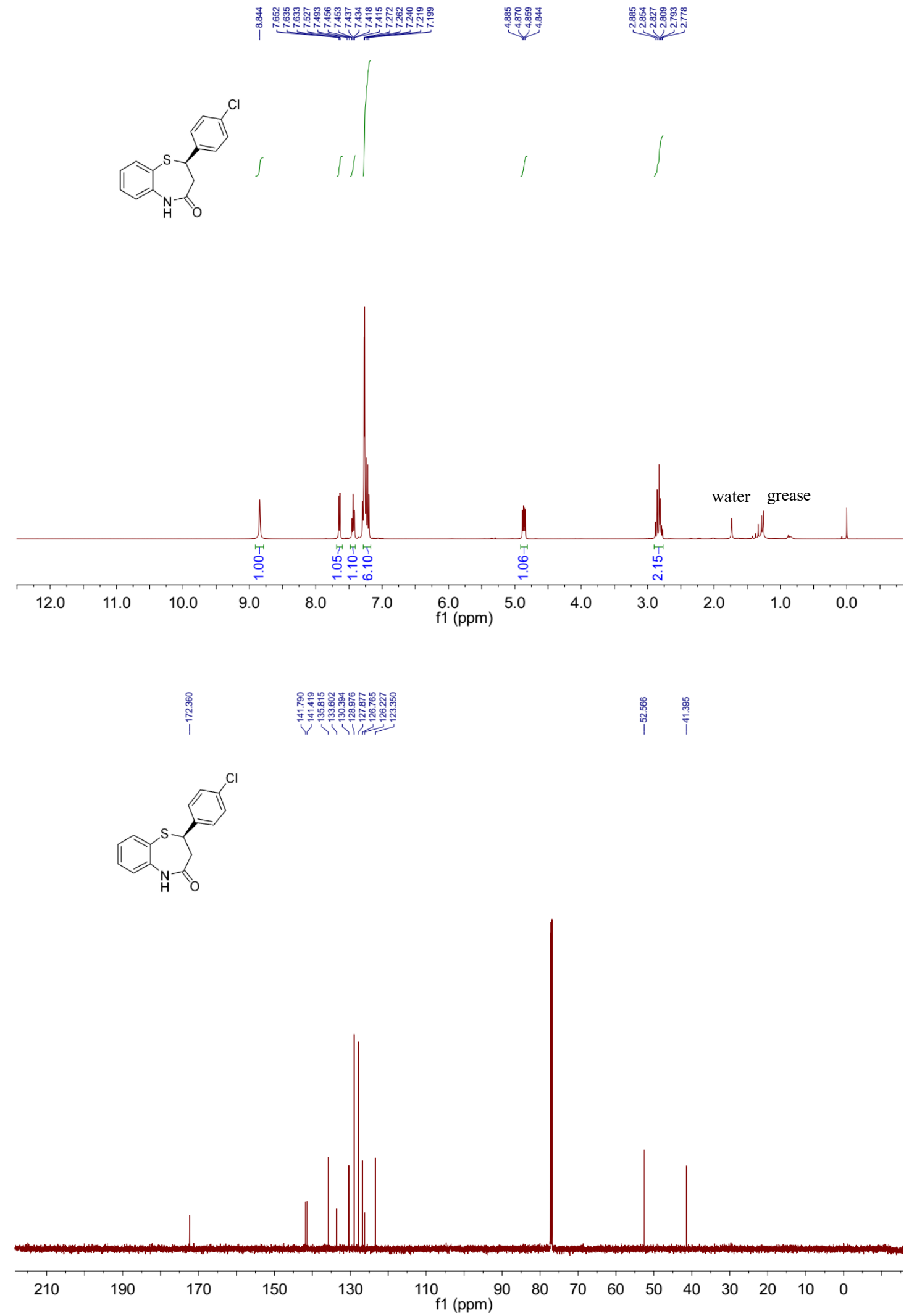
(R)-2-(4-(trifluoromethyl)phenyl)-2,3-dihydrobenzo[b][1,4]thiazepin-4(5H)-one $\mathbf{2 k}$

\section{总}
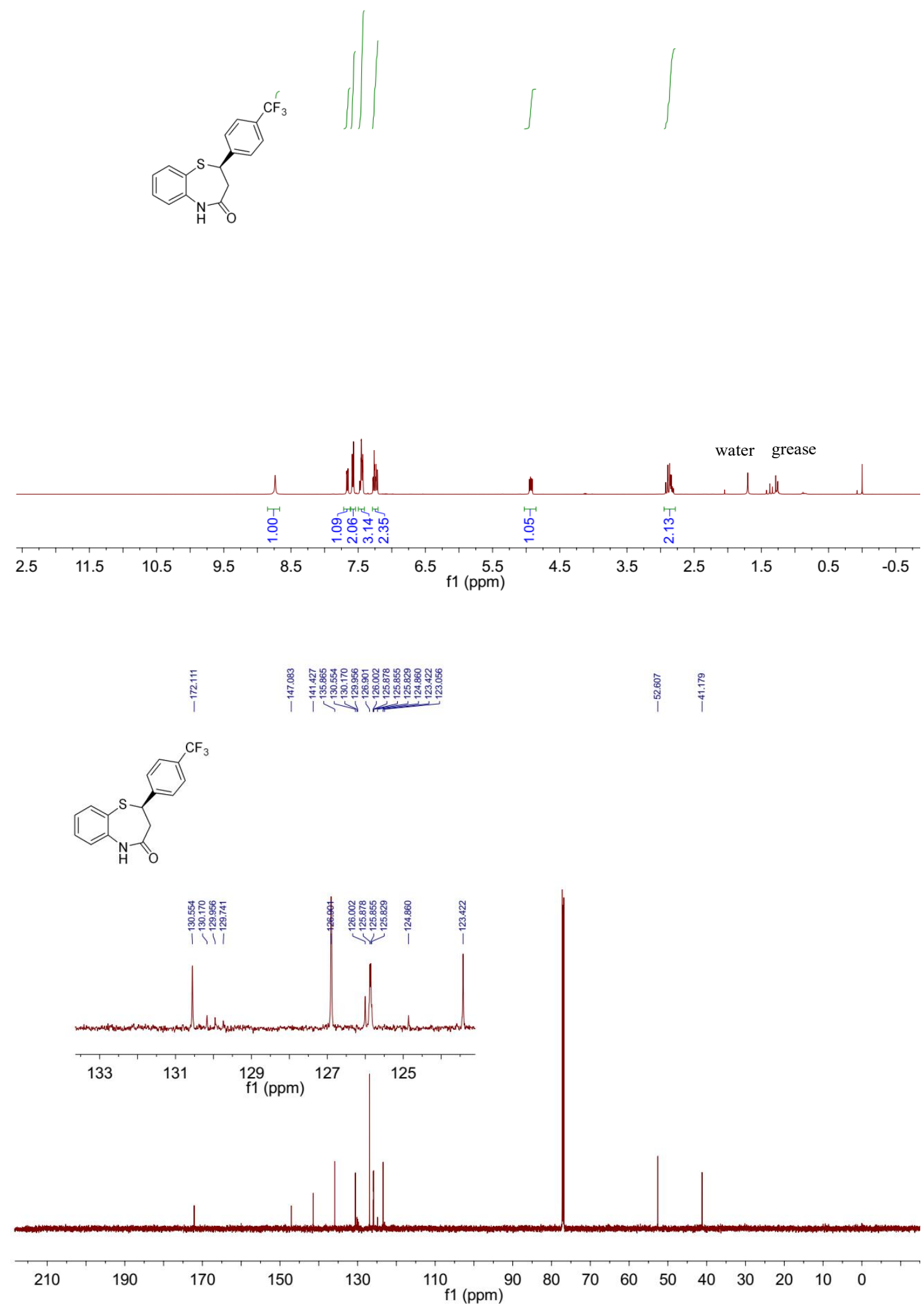
methyl $(R)$-4-(4-oxo-2,3,4,5-tetrahydrobenzo[b][1,4]thiazepin-2-yl)benzoate $2 \mathbf{l}$

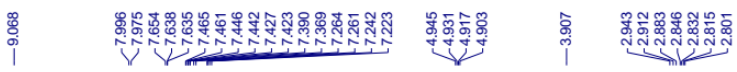<smiles>CC(=O)c1ccc(C2CC(=O)Nc3ccccc3S2)cc1</smiles>
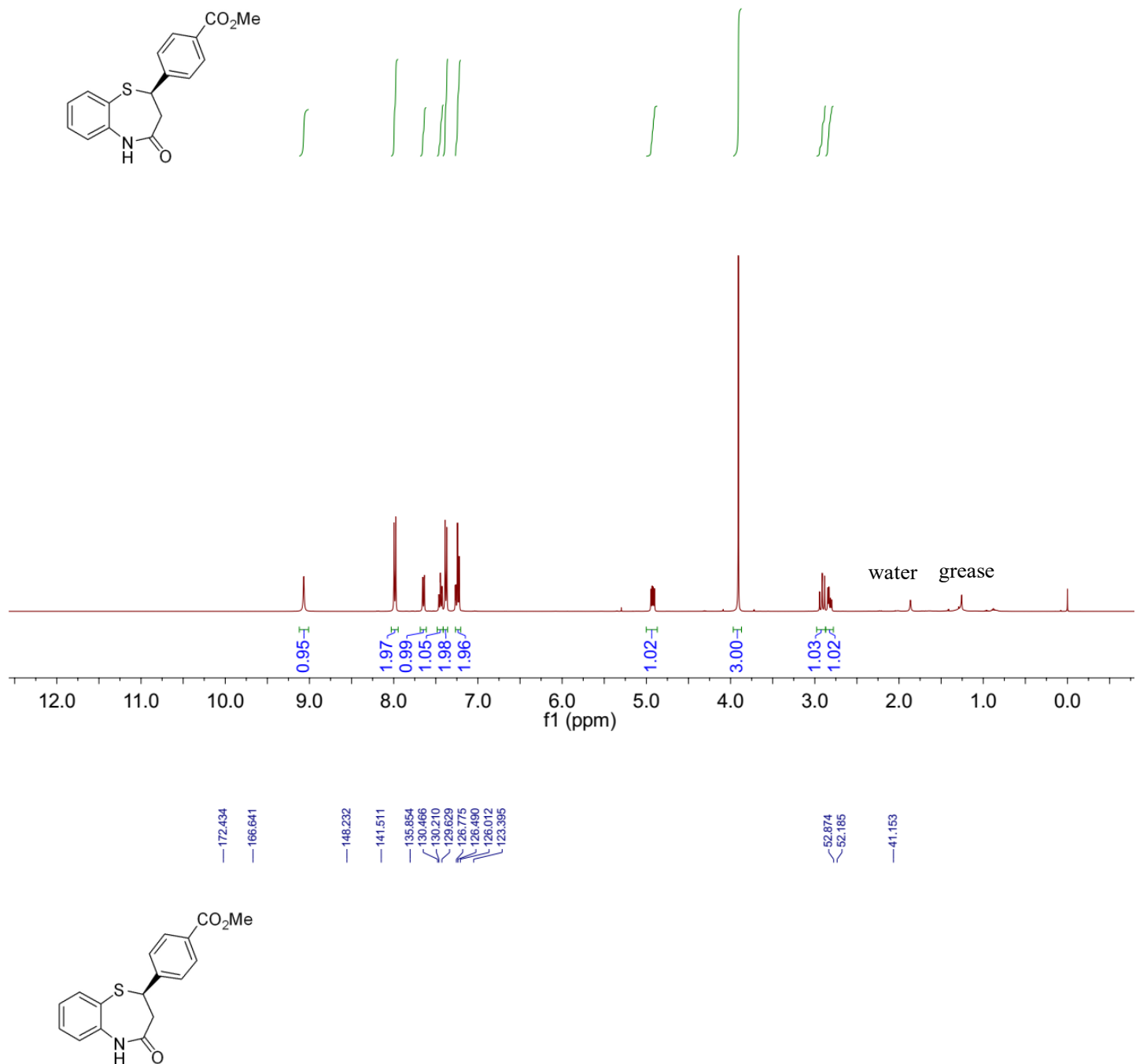

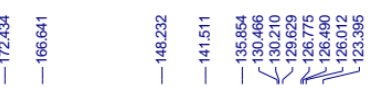

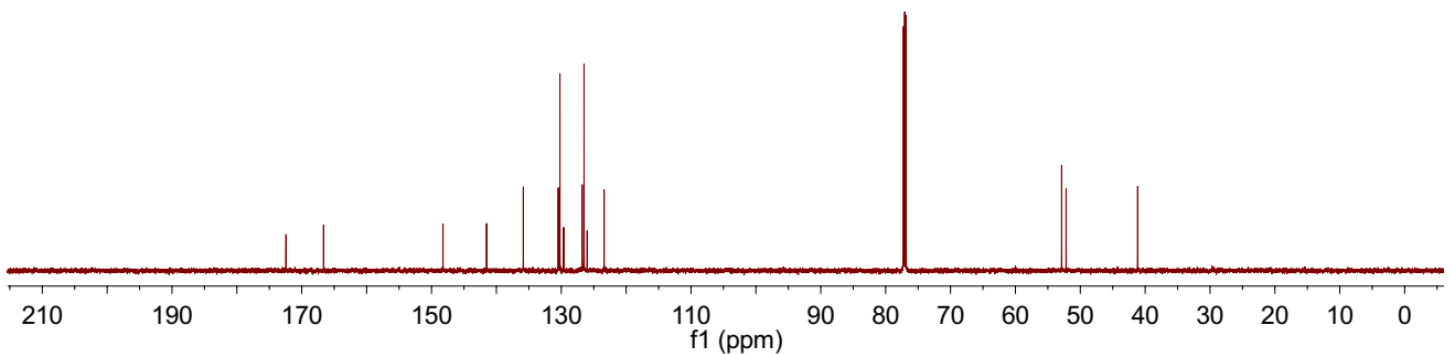


(R)-2-(4-methoxyphenyl)-2,3-dihydrobenzo[b][1,4]thiazepin-4(5H)-one $\mathbf{2 m}$
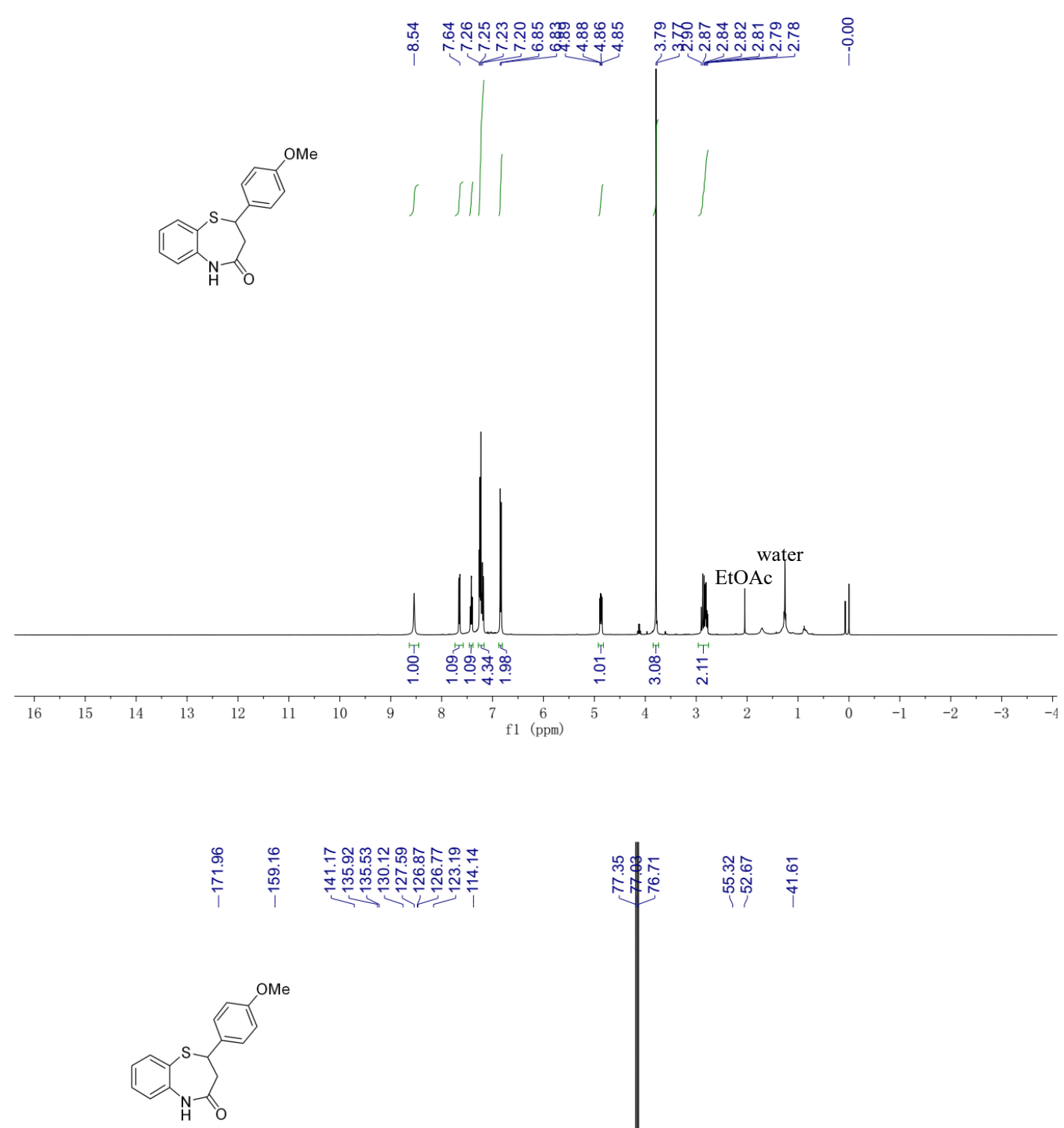

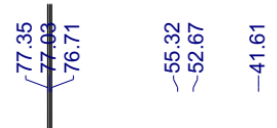

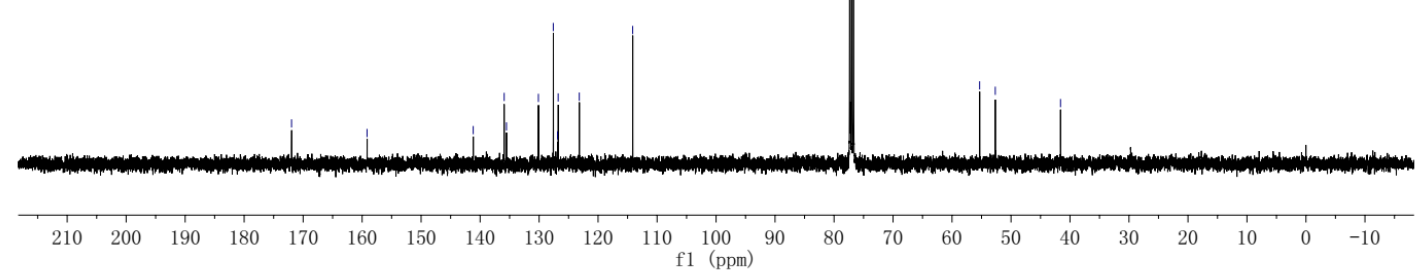


(R)-7-chloro-2-(4-methoxyphenyl)-2,3-dihydrobenzo[b][1,4]thiazepin-4(5H)-one 2n
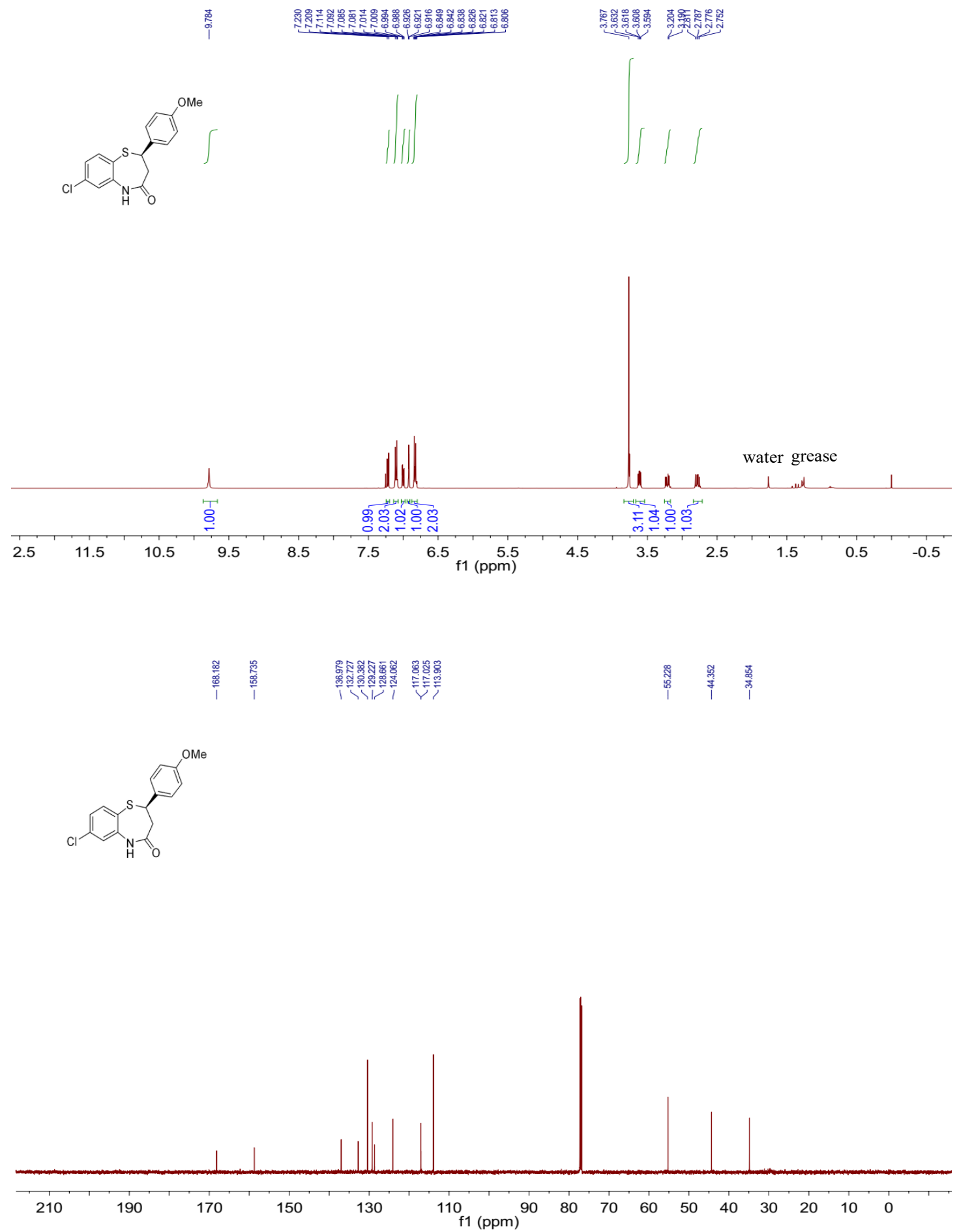
(S)-2-methyl-2,3-dihydrobenzo[b][1,4]thiazepin-4(5H)-one 20
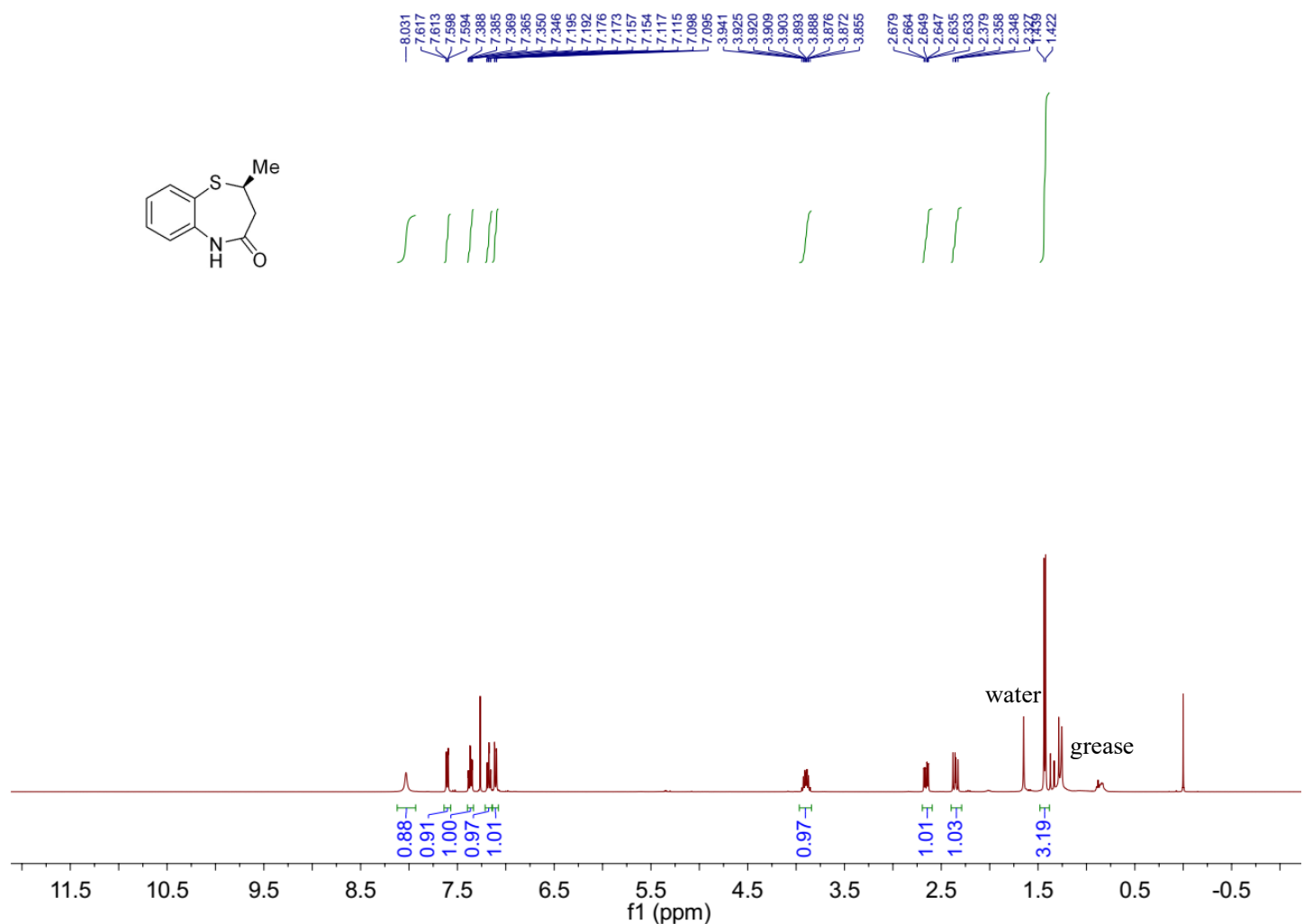<smiles>CC1CC(=O)Nc2ccccc21</smiles>

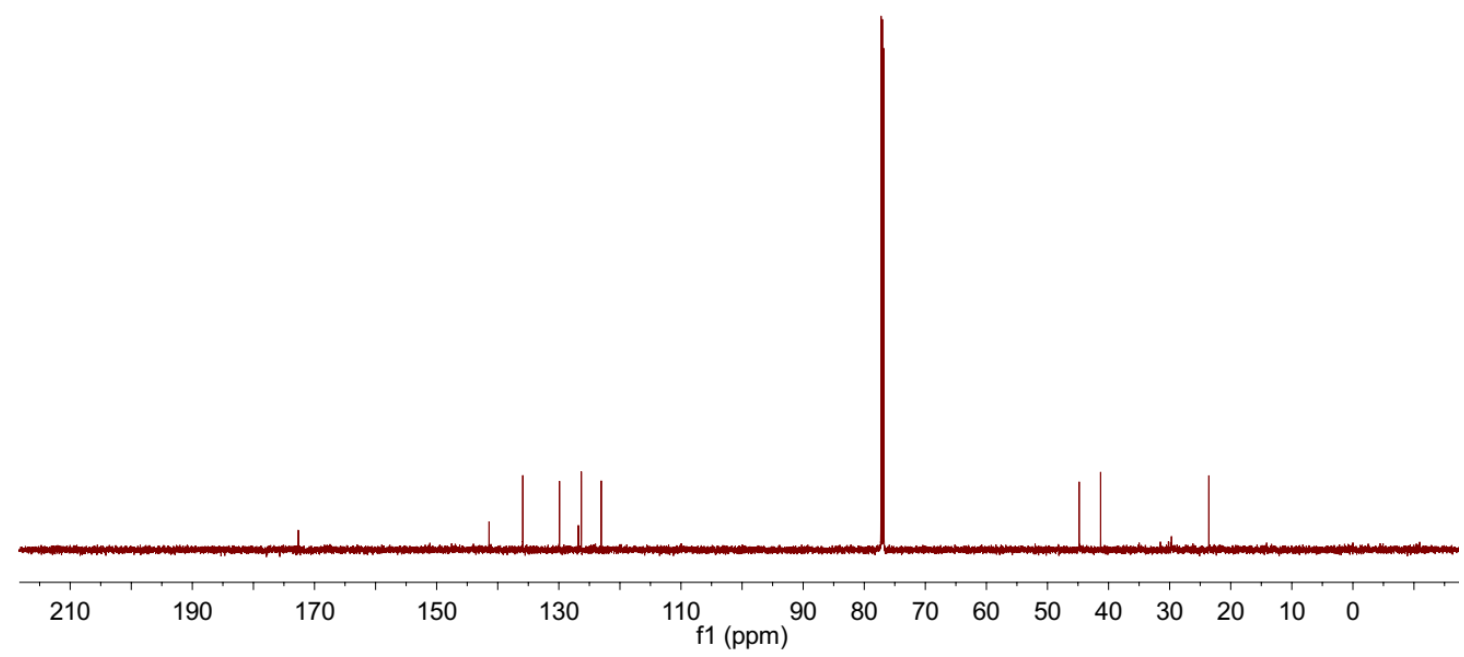


(R)-5-(2-(dimethylamino)ethyl)-2-phenyl-2,3-dihydrobenzo[b][1,4]thiazepin-4(5H)-one 3
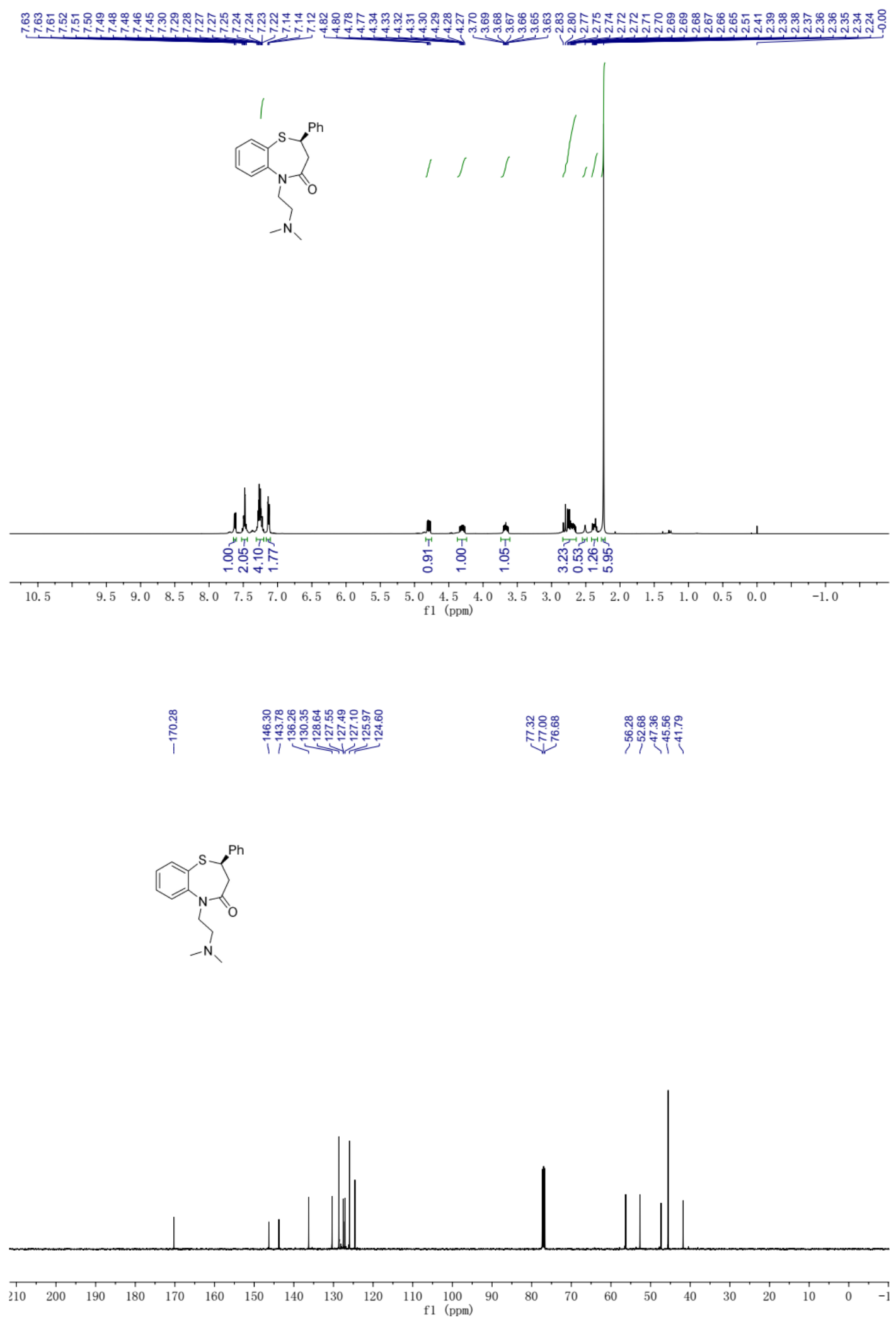


\section{HPLC spectra}

(R)-2-phenyl-2,3-dihydrobenzo[b][1,4]thiazepin-4(5H)-one 2a<smiles>O=C1CC(c2ccccc2)Sc2ccccc2N1</smiles>

Data File d: IChen321.... YANGTAOYYT-4-15CHIRAL $2018-06-02$ 16-20-581001-P2-A1-YT-4-15-RACE.D
Sample Name: YT-4-15-RACE

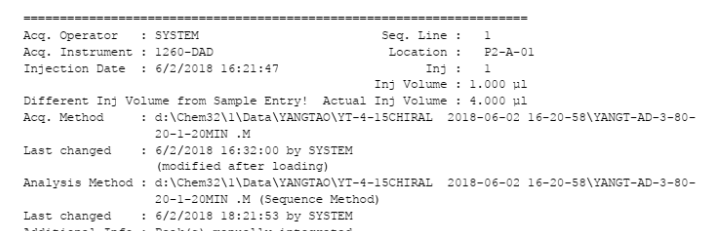

Last changed : $6 / 2 / 2018$ 18:21:53 by SYSTEM

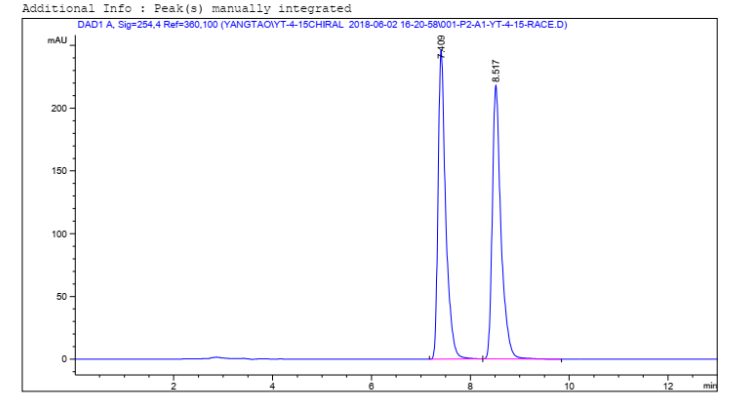

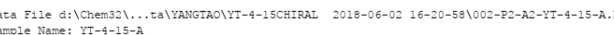

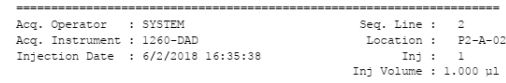

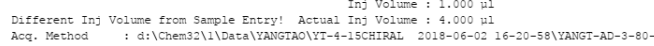

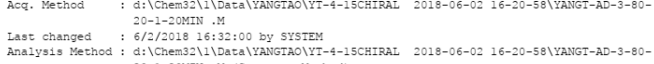

Last changed : 6/2/2018 18:21:53 by SYSTEM

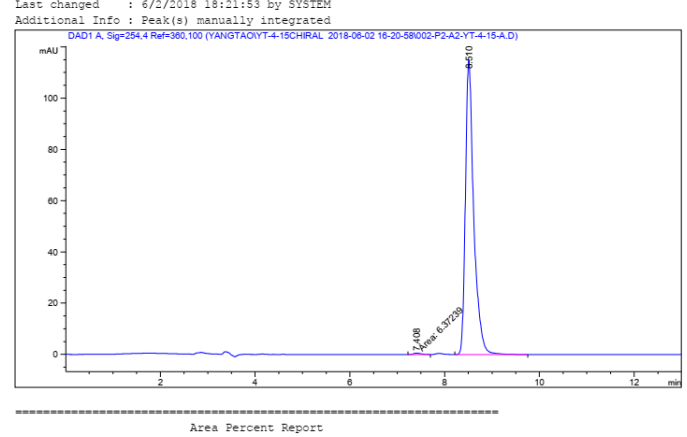

Area Percent Repor

Area Percent Repor
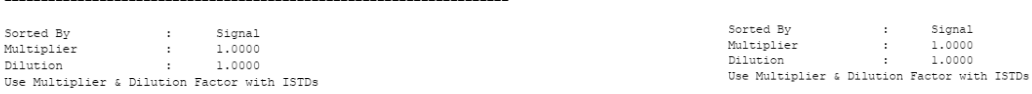

Signai 1: DADI A, Sig $=254,4$ Re $f=360,100$

Signal 1: DAD A, Sig=254, 4 ReE $=360,100$

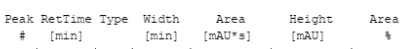

$\begin{array}{lllll}1 & 7.409 \text { BB } & 0.1597 & 2652.30542 & 246.20695 \\ 2 & 0.517 & 49.8946\end{array}$

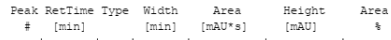

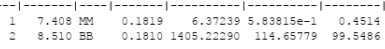

Totals : $\quad 1411.59529 \quad 115.24162$

1260-DAD 11/14/2018 16:18:59 SYSTEM

Page 1 of 2 1260-DAD 11/14/2018 16:19:57 SYSTEM 
(R)-5-methyl-2-phenyl-2,3-dihydrobenzo[ $b][1,4]$ thiazepin-4(5H)-one $\mathbf{2 b}$<smiles>CN1C(=O)CC(c2ccccc2)Sc2ccccc21</smiles>

Data File D: IChem321.... IYYCCIYCC-20191227-3 2019-12-27 20-37-89|ø19-P1-E2-YT-4-95-1-RAC1.D

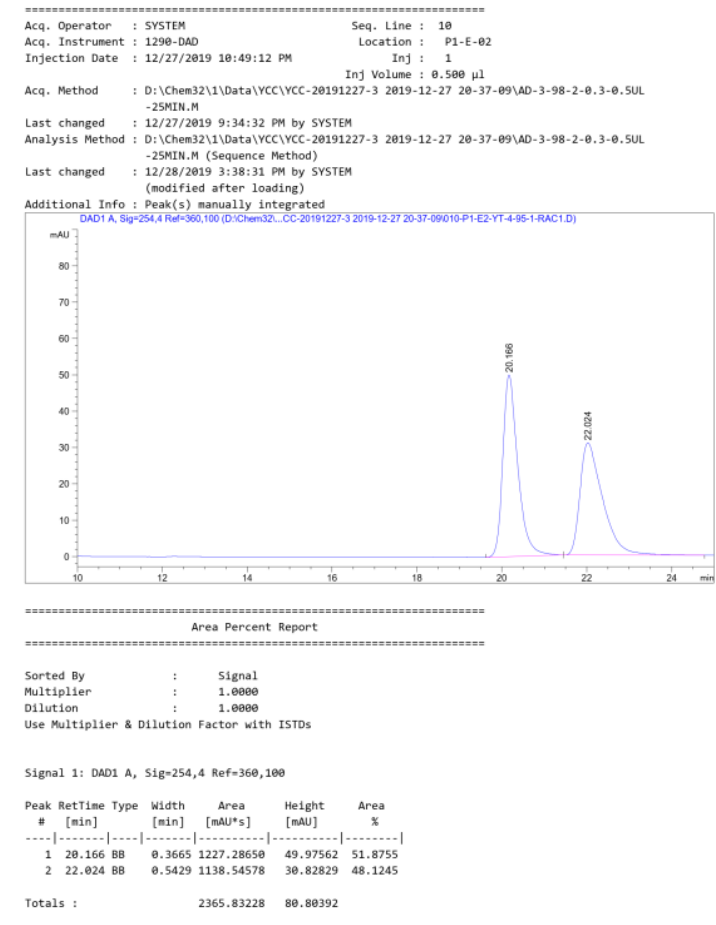

Data File D:IChen3211 1 Data IYCCIYCC-20191227-3 2019-12-27 20-37-99|611-P1-E1-YT-4-95-1.D

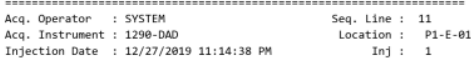

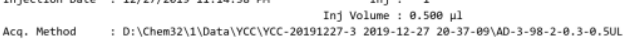

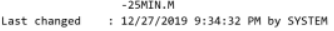

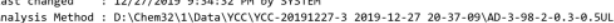

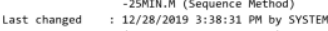
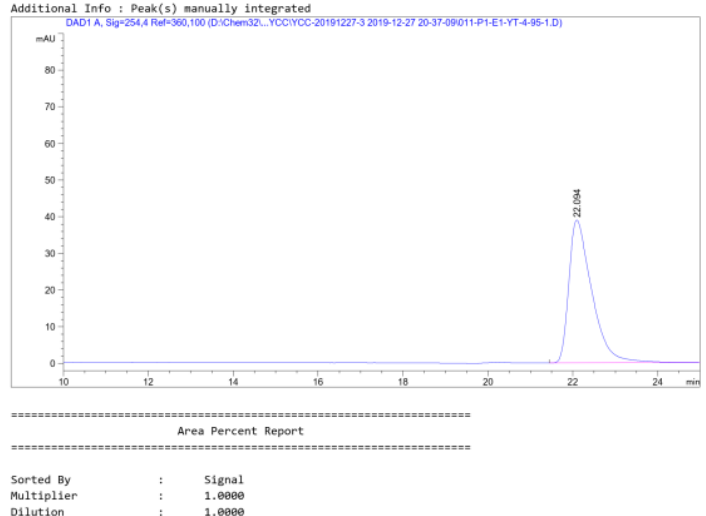

Use Multiplier \& Dilution Factor with ISTD

Signal 1: DAD1 A, Stg-254,4 Ref-36e, 100

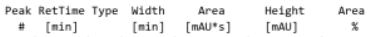

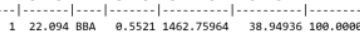

Tota1s : $\quad 1462.75964 \quad 38.94936$

1299-DAD 12/28/2019 3:38:36 PM 5YST 
(R)-5-benzyl-2-phenyl-2,3-dihydrobenzo[ $b][1,4]$ thiazepin-4(5H)-one 2c<smiles>O=C1CC(c2ccccc2)Sc2ccccc2N1Cc1ccccc1</smiles>

Data File d: IChem3211 DatalYaMGTAOYYT-4-95 2018-09-06 17-40-11 1001-P1-F1-YT-4-91-2.D

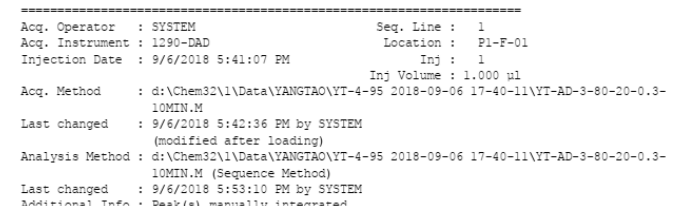
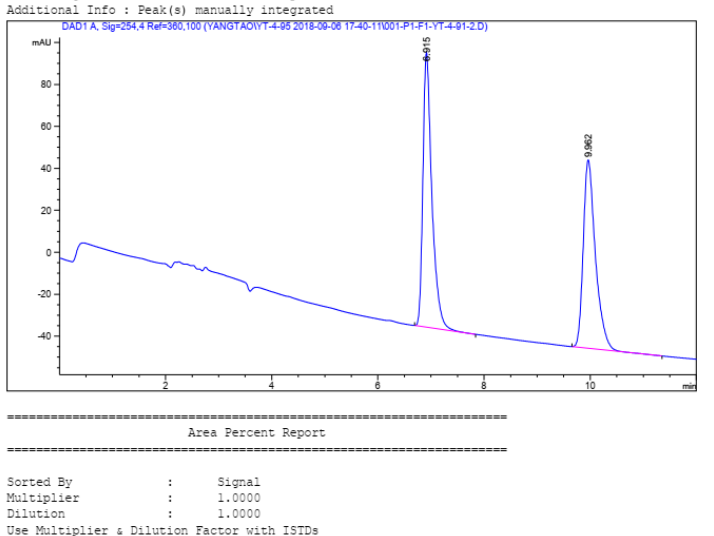

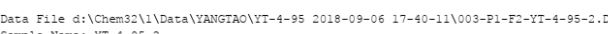

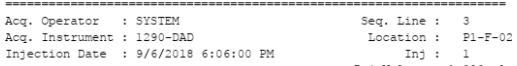

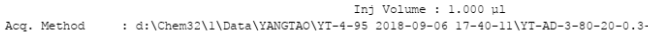

Last changed $\quad \begin{aligned} & 10 M T N . M \\ & 9 / 6 / 2018 \\ & 5: 42: 36 \text { PM by SYSTEM }\end{aligned}$

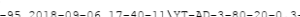

Last changed : 9/6/2018 5:53:10 PM by SYSTE
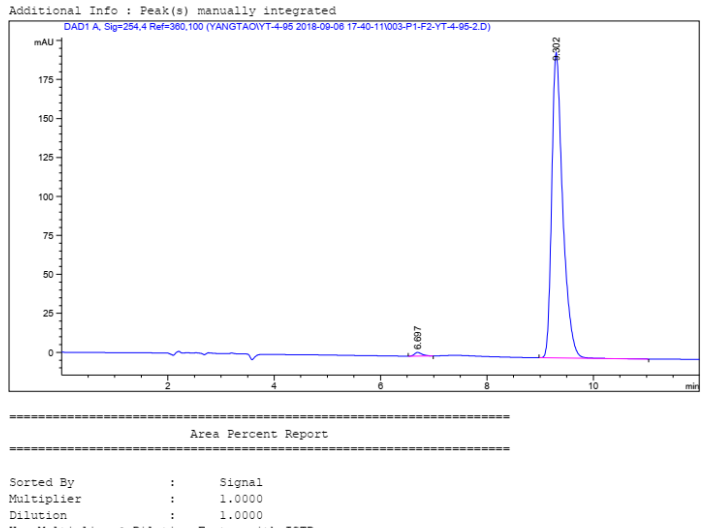

se Nultiplier a Dilution Factor with ISTD

51gnal 1: DAD1 A, S1g $=254,4$ Ref $=360,100$

Signa1 1: DAD A, Sig-254,4 Ref-360, 100

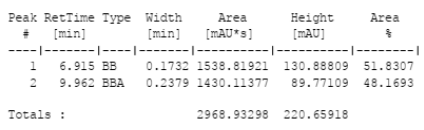

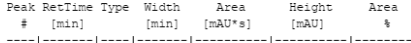

$\begin{array}{rrrrrr}1 & 6.697 \mathrm{BB} & 0.1501 & 23.25039 & 2.33735 & 0.8196 \\ 2 & 9.302 \mathrm{BBA} & 0.2137 & 2813.46509 & 195.73946 & 99.1804\end{array}$

Totals : $\quad 2836.71548 \quad 198.07681$ 
(R)-7-fluoro-2-phenyl-2,3-dihydrobenzo[b][1,4]thiazepin-4(5H)-one 2d<smiles>O=C1CC(c2ccccc2)Sc2ccc(F)cc2N1</smiles>

Data File d: (Chen32) IDatalYaAGGaOYYT-4-95 2018-09-06 17-40-111004-P1-F3-YT-4-91-4.D

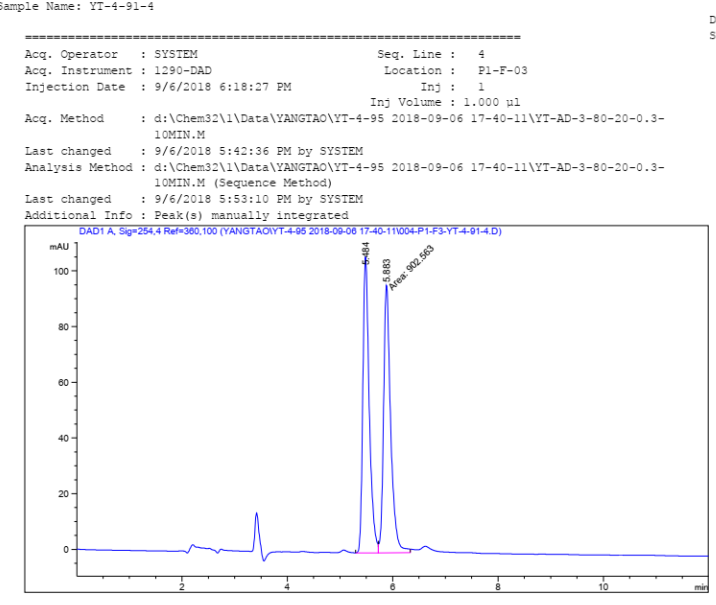

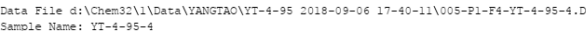

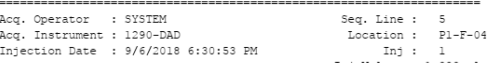

Inj Volune: 1.000 pl
Acq. Method

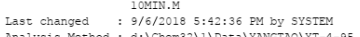

$\begin{array}{lll}\text { Last changed } & \text { 10MTNM M (Sequence Nethod) } \\ 9 / 6 / 2018 & 5: 53: 10 \text { PM by SYSTEM }\end{array}$

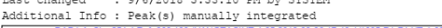

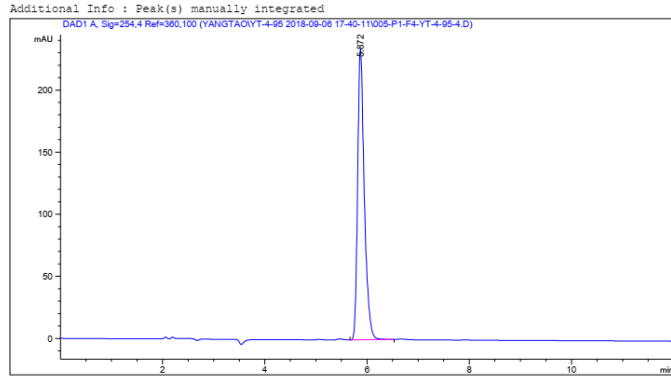

$=0+$ Area Percent Report

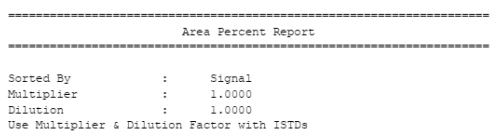

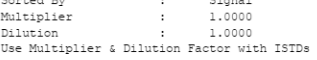

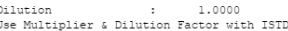

Signal 1: DADI A, Sig $=254,4$ Re $=3600,100$

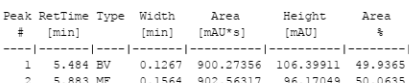

Signal 1) DADI A, Sig $=254,4$ Ref $=360,100$

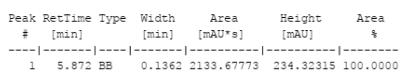

Tota1s : $\quad 1802.83673 \quad 202.56960$

Tota1s

$2133.67773 \quad 234.32315$

1290-DAD 11/14/2018 4:30:28 FM SYSTEM

Page 1 or 2 1290-DAD 11/14/2018 4:29:10 PM SYSTEY 
(R)-7-chloro-2-phenyl-2,3-dihydrobenzo[b][1,4]thiazepin-4(5H)-one $2 \mathbf{e}$<smiles>O=C1CC(c2ccccc2)Sc2ccc(Cl)cc2N1</smiles>

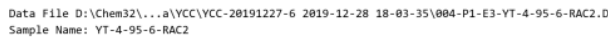

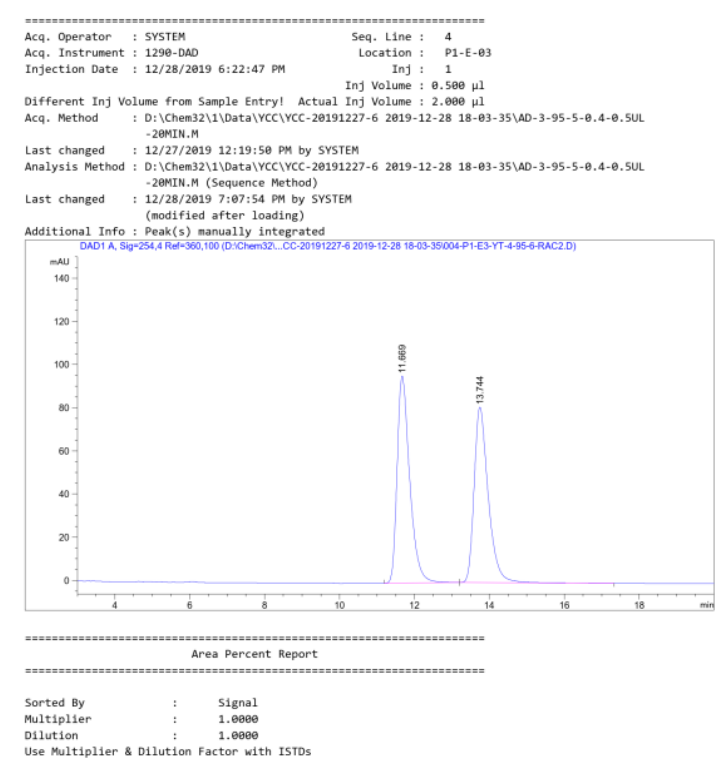

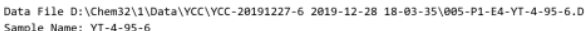

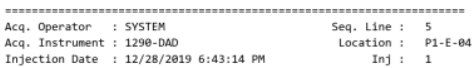

Acq. Instrument : 1298-0⿰00,

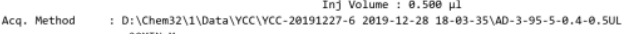

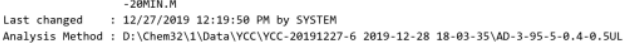

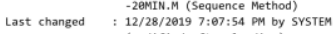
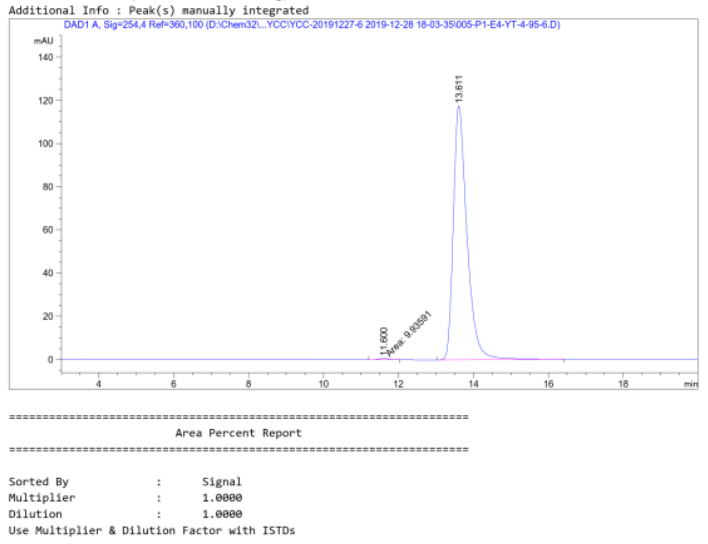

S1gnal 1: DAD1 A, S1g $=254,4$ Reff 366,100

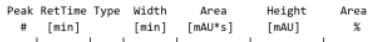

Signal 1: DAD1 A, Sig-254,4 Ref=36e, 100

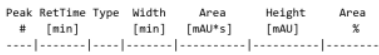

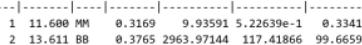

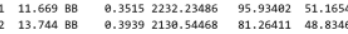

Totals :

$2973.98735 \quad 117.94130$

1299-DA0 12/28/2019 7:88:01 PM SYSTEM 
(R)-7-methyl-2-phenyl-2,3-dihydrobenzo[b][1,4]thiazepin-4(5H)-one $\mathbf{2 f}$<smiles>Cc1ccc2c(c1)NC(=O)CC(c1ccccc1)S2</smiles>

Data File d: IChem32111Datal YANGTAOIYT-4-95 2018-09-06 17-40-11 1006-P1-F5-YT-4-91-5.D
Sample Name: YT-4-91-5

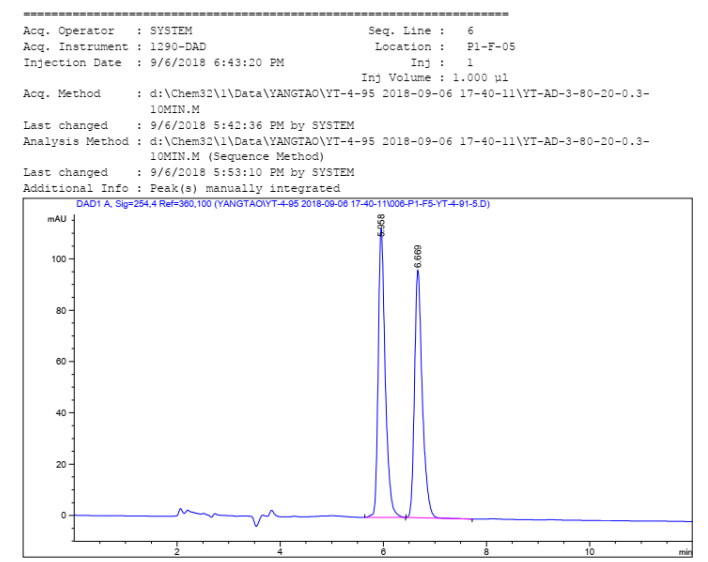

Data File d:IChem3211 Datal YangraolyT-4-95 2018-09-06 17-40-11 1007-P1-56-YT-4-95-5.
Sample Vare: YT-4-95-5

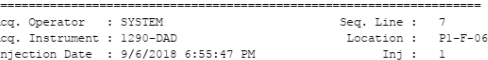

Injection Date : 9/6/2018 6:55:47 PM

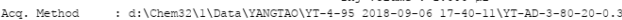

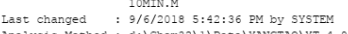

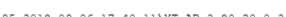

10uril.M (Secuence Method)

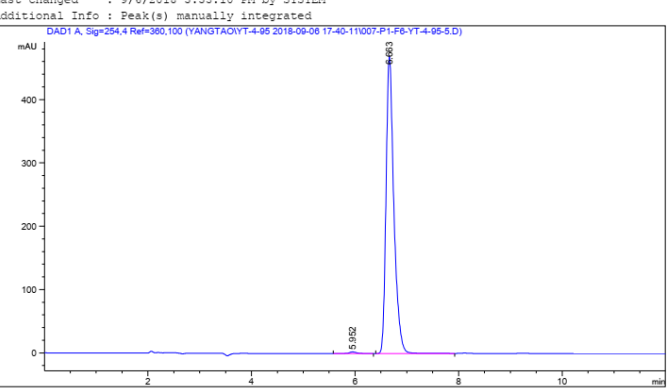

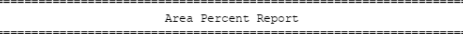
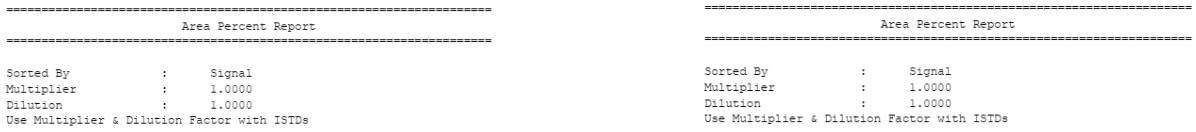

Signal 1: DADI A, Sig $=254,4$ Ret $=360,10$

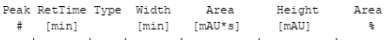

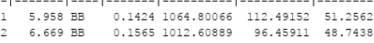

Dota1s : $\quad 2077.40955 \quad 208.95063$

Signal 1: DADI A, Sig $=254,4$ Ref $=360,100$

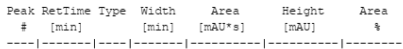

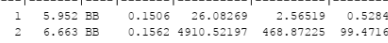

Tota15: $\quad 4936.60466 \quad 471.4374$ 
(R)-6-methyl-2-phenyl-2,3-dihydrobenzo[ $b][1,4]$ thiazepin-4(5H)-one $\mathbf{2 g}$<smiles>Cc1cccc2c1NC(=O)CC(c1ccccc1)S2</smiles>
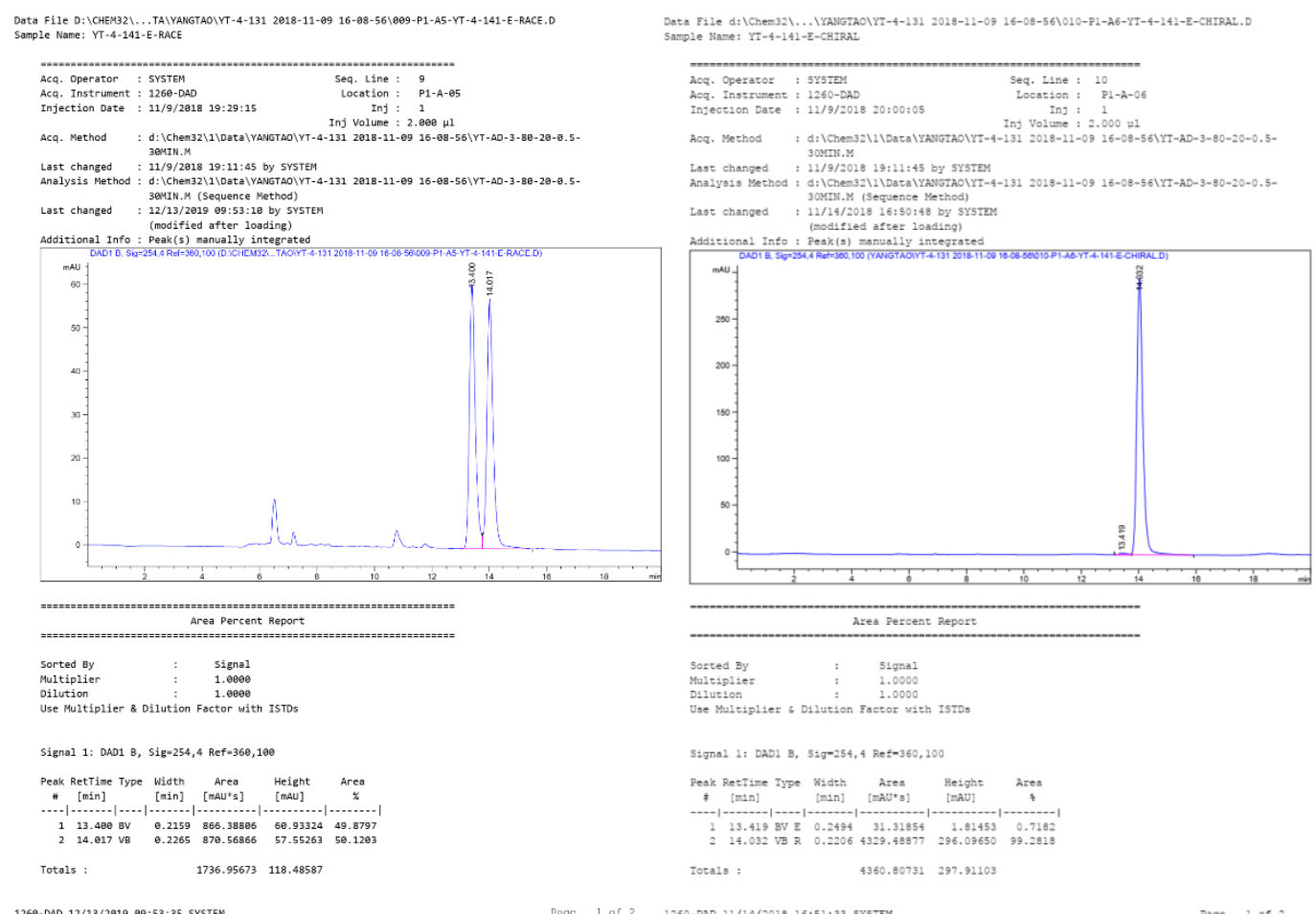

1266-DAD 12/13/2019 99:53:35 SYSTEM 
(R)-8-methoxy-2-phenyl-2,3-dihydrobenzo[b][1,4]thiazepin-4(5H)-one $\mathbf{2 h}$<smiles>COc1ccc2c(c1)SC(c1ccccc1)CC(=O)N2</smiles>

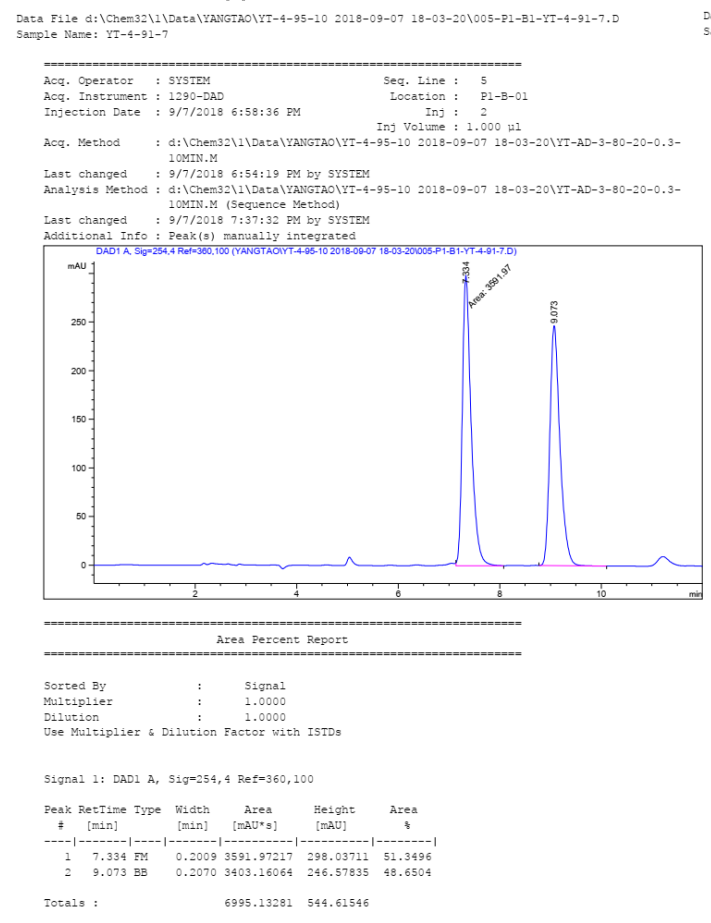

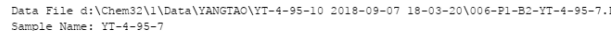

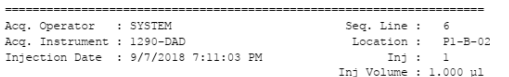

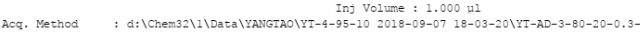
IOMTN.M

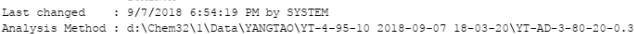

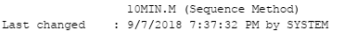
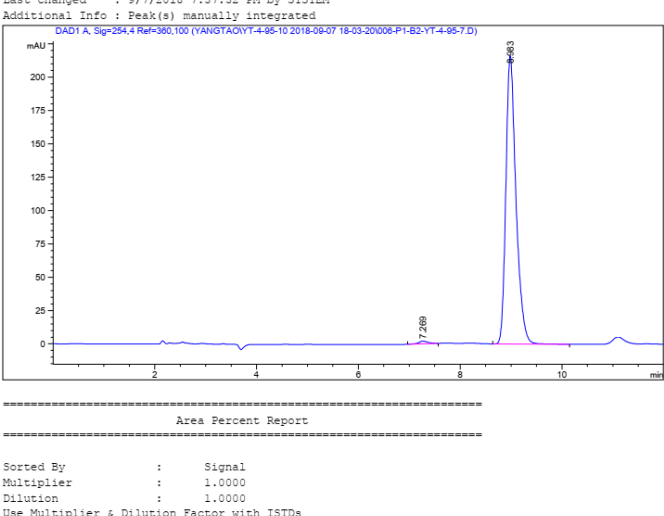

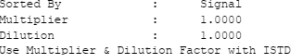

Signal 1: DADI A, Sig $=254,4$ Re $=360,100$

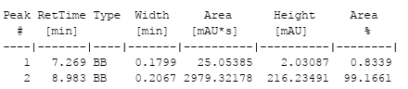

Tota1s : $\quad 3004.37563 \quad 218.26577$

1290-DAD 11/14/2018 4:33:22 PM SYSTEM

Page 1 of 2 1290-DAD 11/14/2018 4:33:45 FM SYSTEM

Fage 1 of 2 
(R)-2-(4-fluorophenyl)-2,3-dihydrobenzo[b][1,4]thiazepin-4(5H)-one $\mathbf{2 i}$<smiles>O=C1CC(c2ccc(F)cc2)Sc2ccccc2N1</smiles>
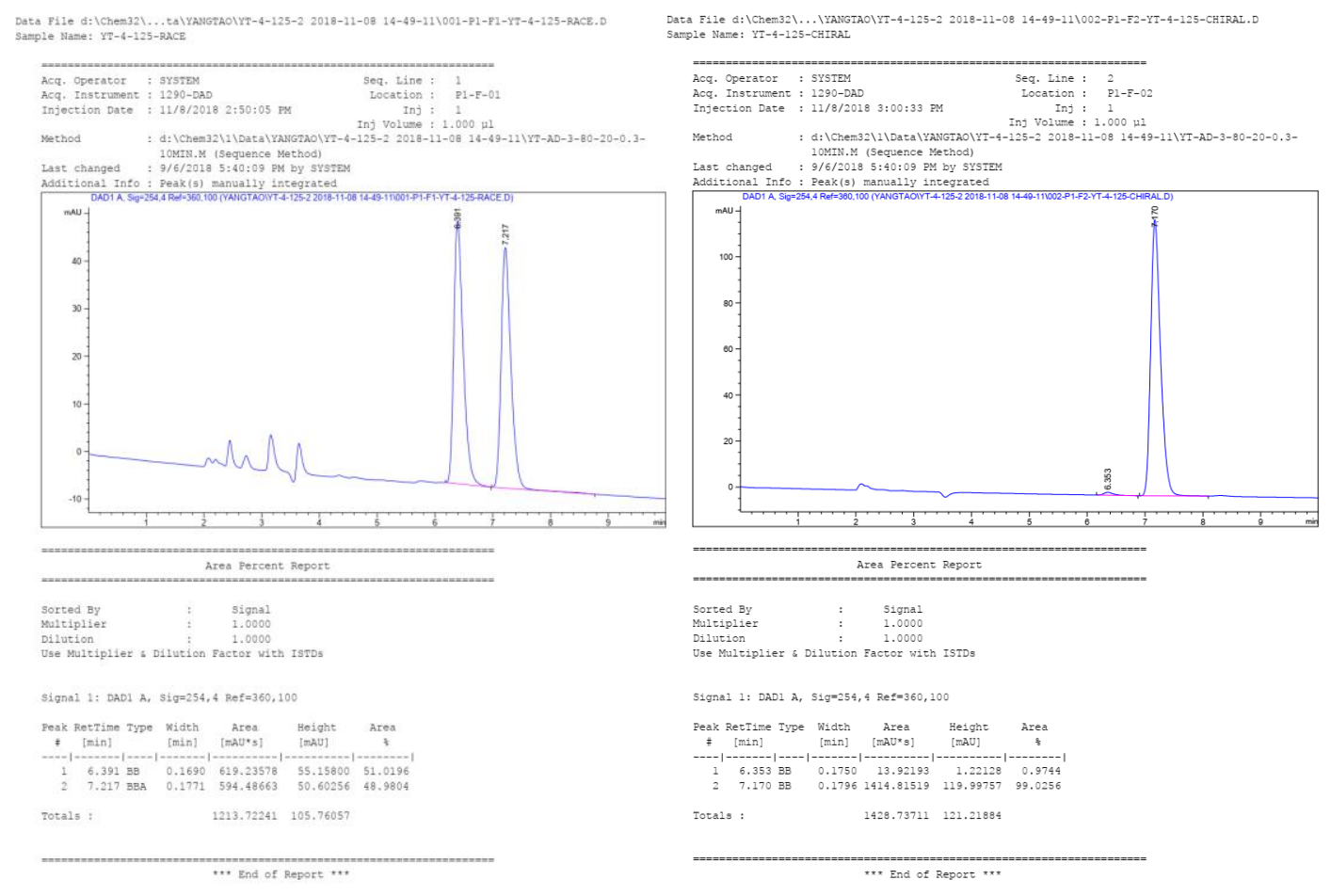
(R)-2-(4-chlorophenyl)-2,3-dihydrobenzo[b][1,4]thiazepin-4(5H)-one $\mathbf{2 j}$<smiles>O=C1CC(c2ccc(Cl)cc2)Sc2ccccc2N1</smiles>

Data File d:IChem3211 Datal YaMGTaO YYT-4-131 2018-11-09 16-08-56|001-P1--A1-YT-4-131-RACE.D

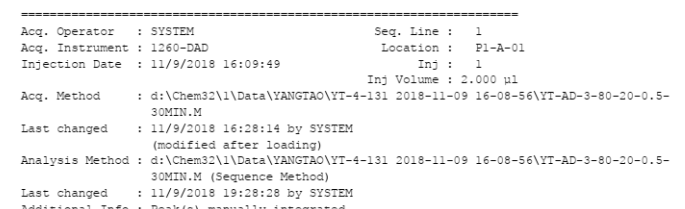

Last changed : 1 I

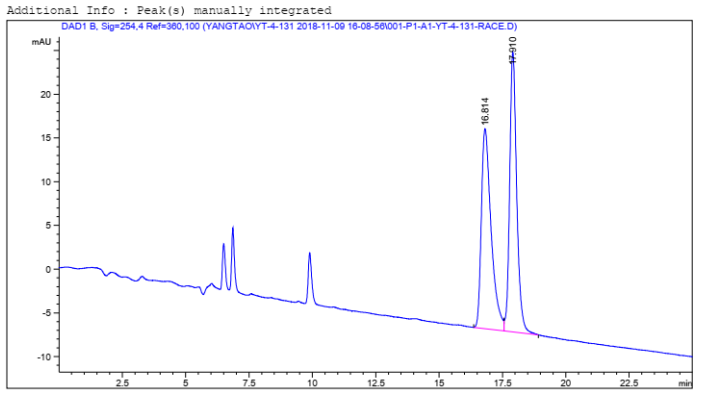

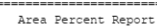

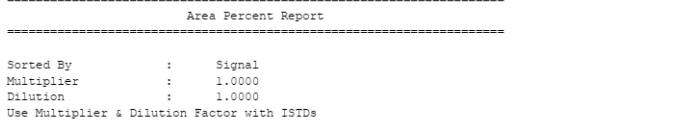

Signai 1: DAD1 B, Sig $=254,4$ Ref $=360,100$

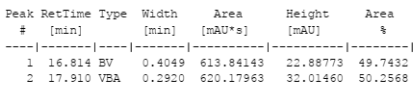

Tota1s : $\quad 1234.02106 \quad 54.90233$

1260-DAD 11/14/2018 16:47:27 SYSTEM

Page 1 of 2 1260-DAD 11/14/2018 16:48:13 SYSTEM

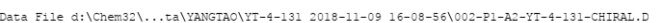

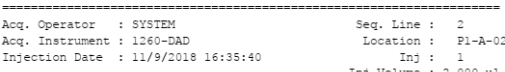

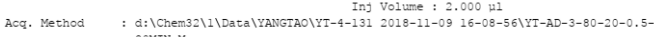

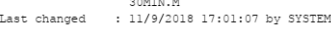

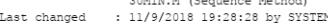

Last changed : 11/9/2018 19:28:28 by SYSTE

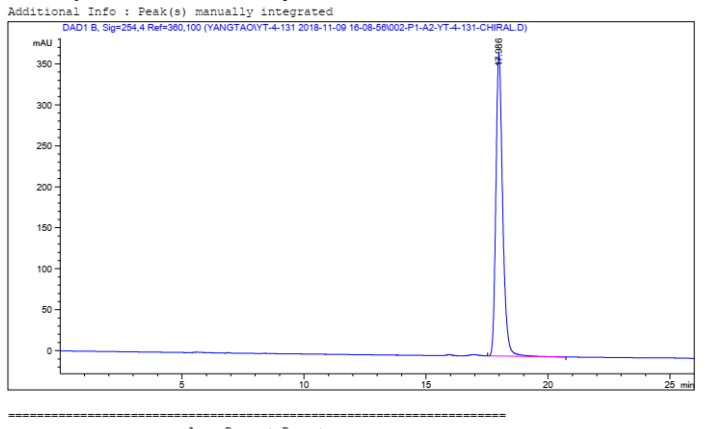

Area Percent Report

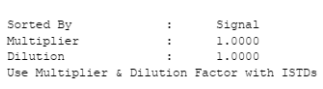

Signal 1: DADI B, Sig $=254,4$ Ref $=360,100$

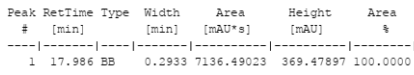

Tota1s : $\quad 7136.49023 \quad 369.4789$ 
(R)-2-(4-(trifluoromethyl)phenyl)-2,3-dihydrobenzo[b][1,4]thiazepin-4(5H)-one $\mathbf{2 k}$<smiles>O=C1CC(c2ccc(C(F)(F)F)cc2)Sc2ccccc2N1</smiles>

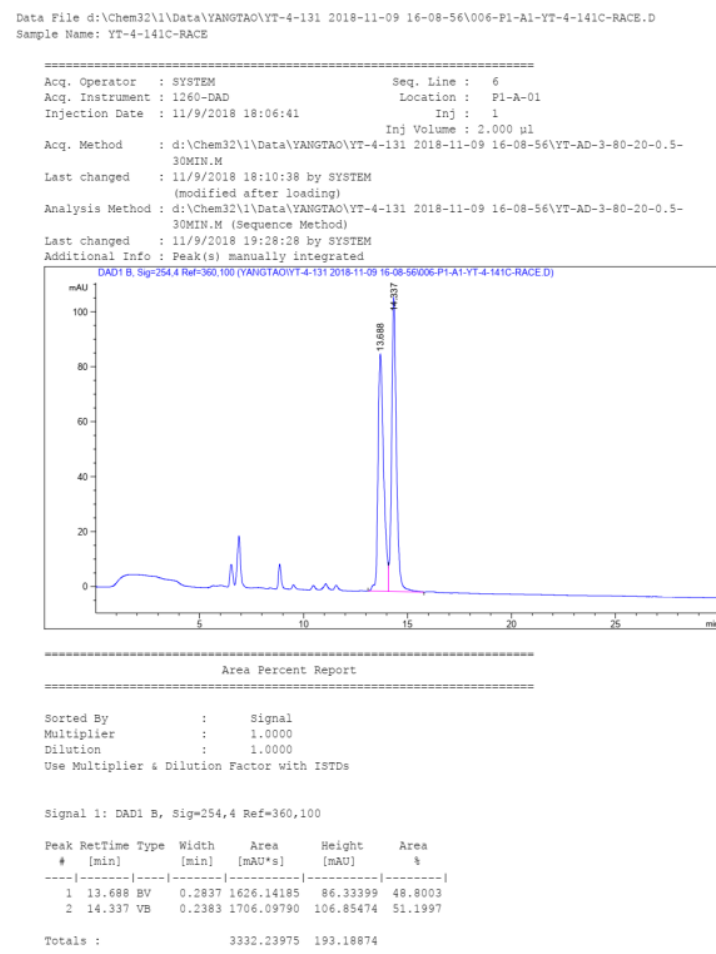

Data File d: IChem321 .... |YAMGTAOIYT-4-131 2018-11-09 16-08-561007-P1-A2-YT-4-141C-CHIRRI.D

\begin{tabular}{|c|c|}
\hline 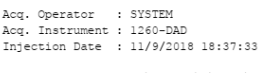 & $\begin{aligned} \text { Sec. Iine } & 7 \\
\text { Iocation } & \text { p1-A-02 } \\
\text { Inj } & 1 \\
\text { Inj Volume } & 2.000 \mathrm{pl}\end{aligned}$ \\
\hline
\end{tabular}

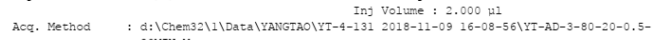

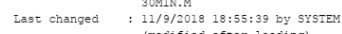

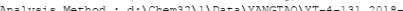

3OMIN.M (Sequence Method)

Last changed : $11 / 9 / 201019.20: 28$ by sYSTE
Additional Info :
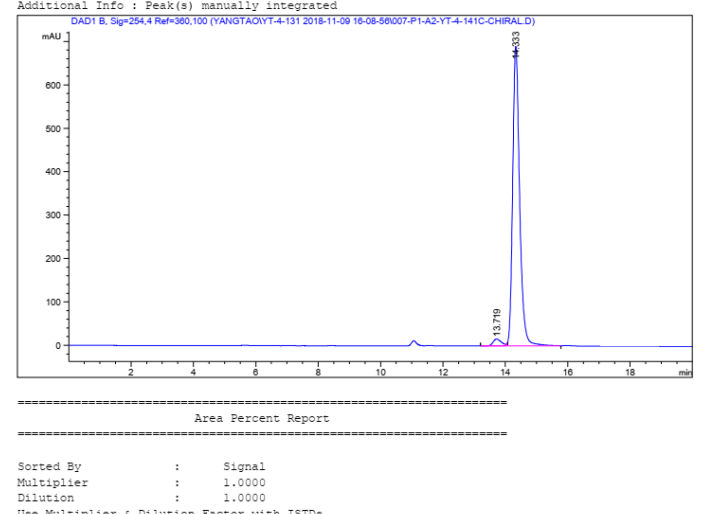

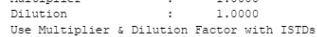

Signal 1: DAD1 B, $5 \mathrm{ig}=254,4$ Re $E=360,100$

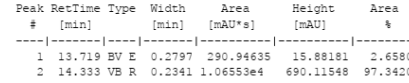

Tota1s : $\quad 1.0946224 \quad 705.99729$ 
methyl $(R)$-4-(4-oxo-2,3,4,5-tetrahydrobenzo[b][1,4]thiazepin-2-yl)benzoate $2 \mathbf{I}$<smiles>CC(=O)c1ccc(C2CC(=O)Nc3ccccc3S2)cc1</smiles>
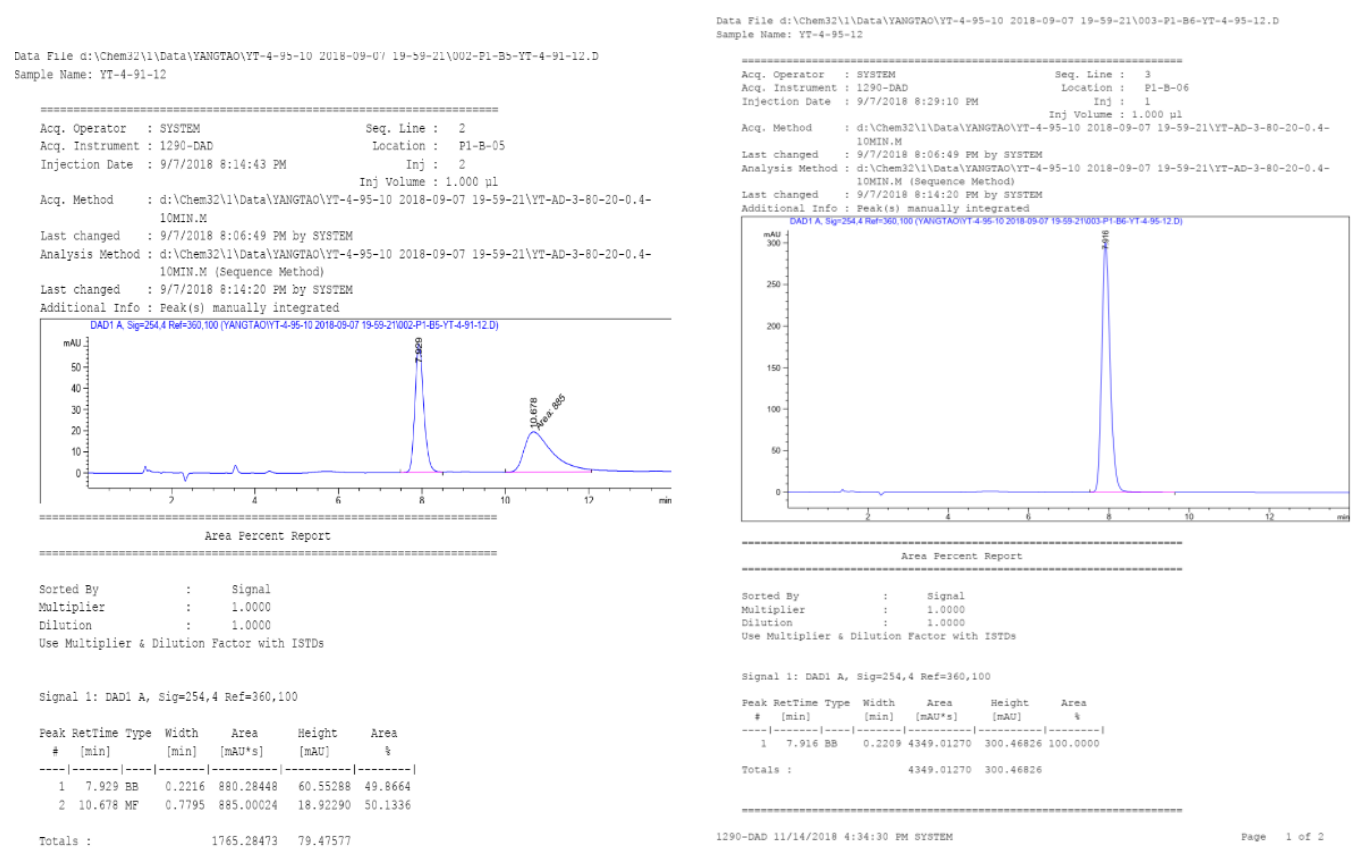
(R)-2-(4-methoxyphenyl)-2,3-dihydrobenzo[b][1,4]thiazepin-4(5H)-one $\mathbf{2 m}$<smiles>COc1ccc([C@@H]2CC(=O)Nc3ccccc3S2)cc1</smiles>
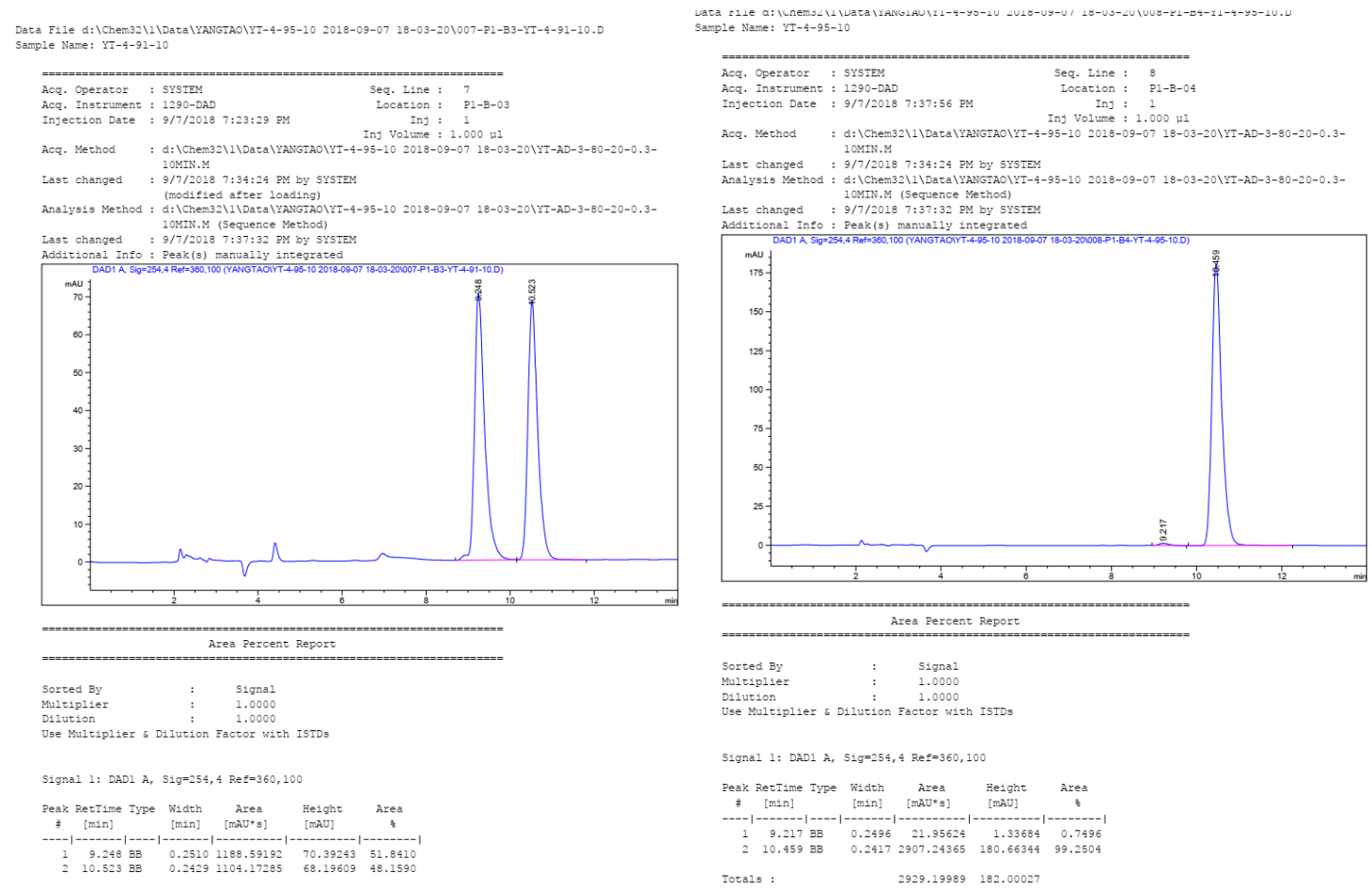
(R)-7-chloro-2-(4-methoxyphenyl)-2,3-dihydrobenzo[b][1,4]thiazepin-4(5H)-one 2n<smiles>COc1ccc(C2CC(=O)Nc3cc(Cl)ccc3S2)cc1</smiles>

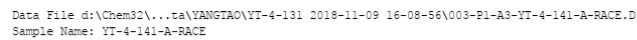
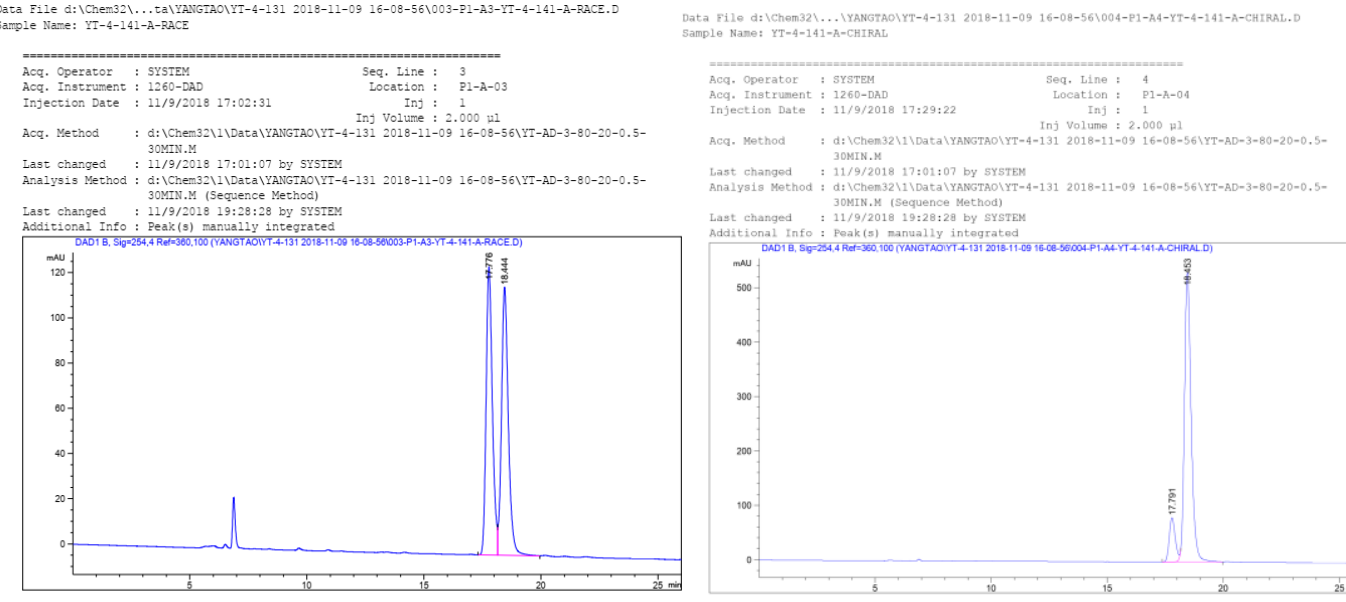

Area Percent Report

atod
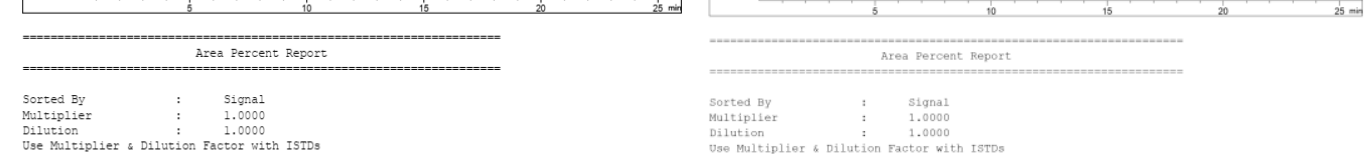

Stgnal 1: DAD1 B, Sig=254,4 Ref=360, 100

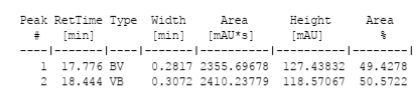

5ignal 1: DAD1 B, Big-254,4 Ref-360, 100

Tota1s : $\quad 4765.93457 \quad 246.00900$

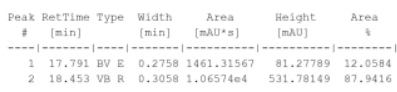

Tota1s : $\quad 1.21187 \mathrm{e4} \quad 613.05939$

1260-DAD 11/14/2018 16:48:34 SYSTEM 
(S)-2-methyl-2,3-dihydrobenzo[b][1,4]thiazepin-4(5H)-one 20<smiles>CC1CC(=O)Nc2ccccc2S1</smiles>

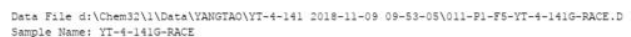

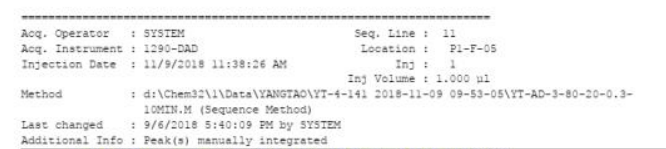

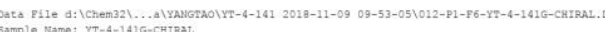

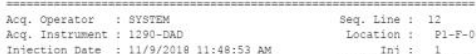

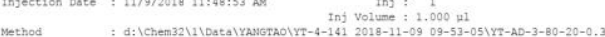

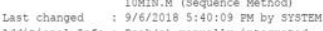

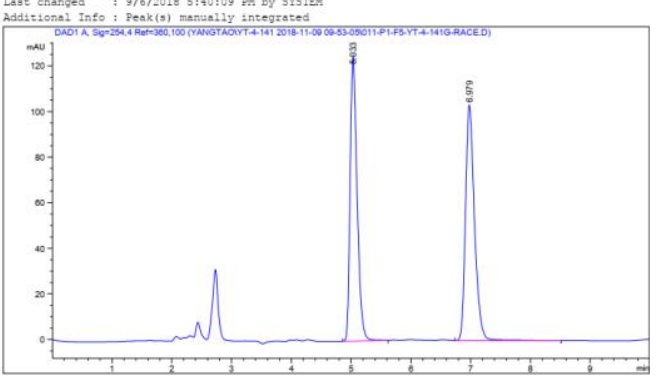

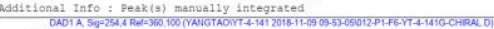

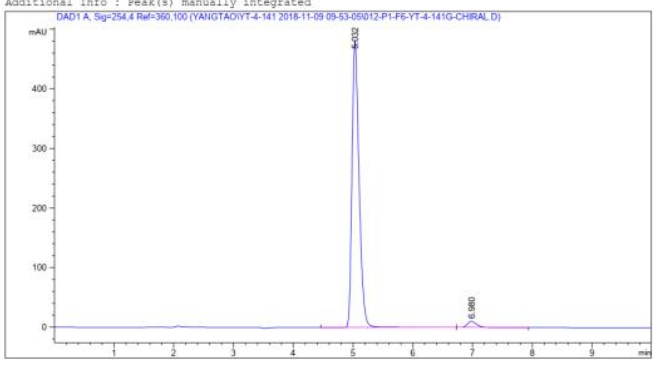

Ares Peercent Report
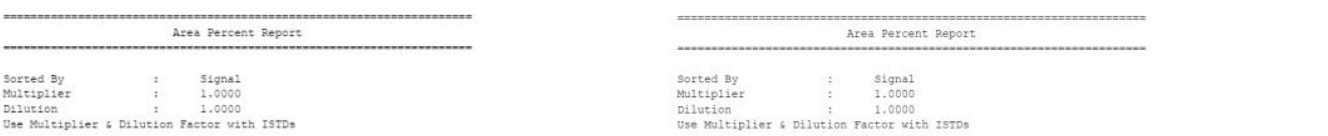

Signal 1: DADI A, S18v254, 4 Ret-360, 100

signal 1: DAD1 A, sig-254,4 Ret-360,100

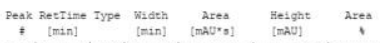

Peak Retrime Type widen Ares
[min]
$[$ [min]

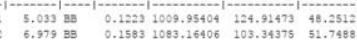

Totais, : $\quad 2093.11810 \quad 228.25848$

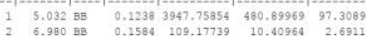

- End of Report $\cdots$

Total

$4056.93594 \quad 491.30932$ 
(R)-5-(2-(dimethylamino)ethyl)-2-phenyl-2,3-dihydrobenzo[b][1,4]thiazepin-4(5H)-one 3<smiles>CN(C)CCN1C(=O)CC(c2ccccc2)Sc2ccccc21</smiles>

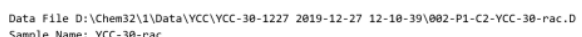

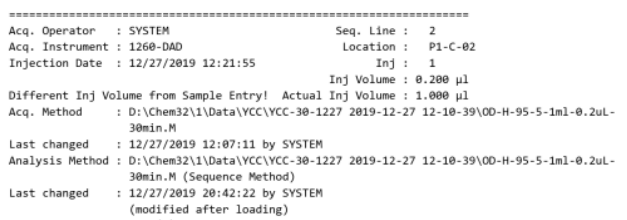

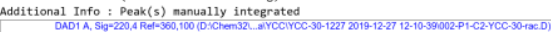

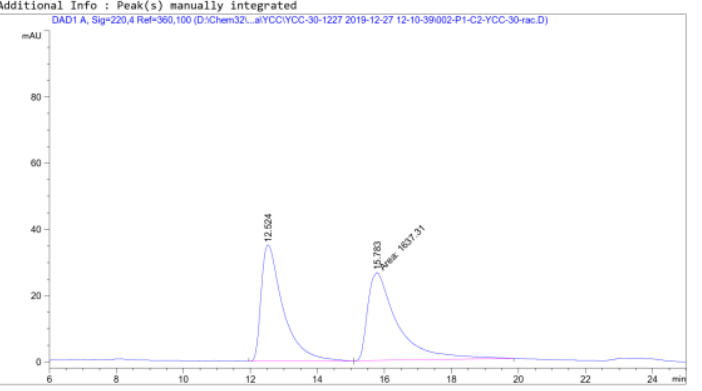

Area Percent Report
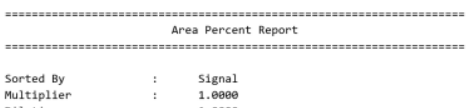

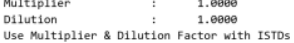

Signal 1: DAD1 A, S1g-22e, 4 Ref $=36 \theta, 100$

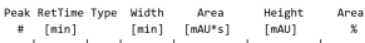

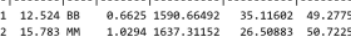

Totals : $\quad 3227.97644 \quad 61.62484$

268-DAD 12/27/2019 28:42:28 SYSTE

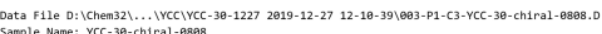

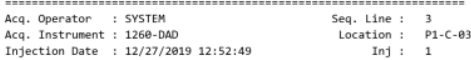

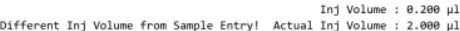

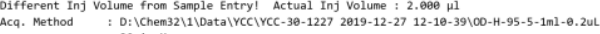

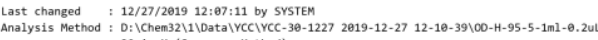

Last changed : 12/27/2019 20:38:5e by SYST

(modified after loading)
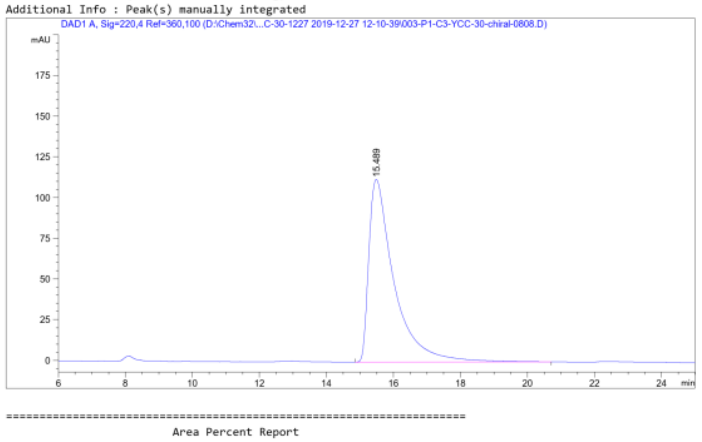

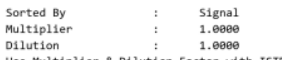

S1gnal 1: DAD1 A, S1g-22e, 4 Ref $=36 \theta, 100$

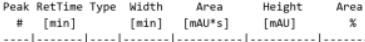

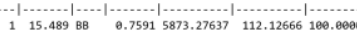

Totals : $\quad 5873.27637 \quad 112.12666$ 


\section{Reference:}

[1] (a) Manfroni, G.; Meschini, F.; Barreca, M. L.; Leyssen, P.; Samuele, A.; Iraci, N.; Sabatini, S.; Massari, S.; Maga, G.; Neyts, J.; Cecchetti, V. Bioorg. Med. Chem. 2012, 20, 866-876; (b) Fukata, Y.; Asano, K.; Matsubara, S. J. Am. Chem. Soc. 2015, 137, 5320-5323.

[2] (a) Park, K.; Heo, Y.; Lee, S., Org. Lett. 2013, 15, 3322-3325; (b) Feng, Q.; Yang, K.; Song, Q. Chem. Commun. 2015, 51, 15394-15397.

[3] Li, W.; Schlepphorst, C.; Daniliuc, C.; Glorius, F. Angew. Chem. Int. Ed. 2016, 55, 33003303.

[4] Ried, W.; Marx, W. Chem. Ber. 1957, 90, 2683-2687.

[5] Fang, X.; Li, J.; Wang, C.-J. Org. Lett. 2013, 15, 3448-3451.

[6] Fang, C.; Lu, T.; Zhu, J.; Sun, K.; Du, D. Org. Lett. 2017, 19, 3470-3473.

[7] Wang, G.; Tang, Y.; Zhang, Y.; Liu, X.; Lin, L.; Feng, X. Chem. Eur. J. 2017, 23, 554-557.

[8] Meninno, S.; Volpe, C.; Lattanzi, A. Chem. Eur. J. 2017, 23, 4547-4550.

[9] Ogawa, T.; Kumagai, N.; Shibasaki, M. Angew. Chem. Int. Ed. 2012, 51, 8551-8554. 